Portland State University

PDXScholar

Spring 6-14-2013

\title{
The Effects of Green Smoothie Consumption on Blood Pressure and Health-Related Quality of Life: A Randomized Controlled Trial
}

Emiko Maeda

Portland State University

Follow this and additional works at: https://pdxscholar.library.pdx.edu/open_access_etds

Part of the Alternative and Complementary Medicine Commons, and the Dietetics and Clinical Nutrition Commons

Let us know how access to this document benefits you.

\section{Recommended Citation}

Maeda, Emiko, "The Effects of Green Smoothie Consumption on Blood Pressure and Health-Related Quality of Life: A Randomized Controlled Trial" (2013). Dissertations and Theses. Paper 974.

https://doi.org/10.15760/etd.974

This Thesis is brought to you for free and open access. It has been accepted for inclusion in Dissertations and Theses by an authorized administrator of PDXScholar. Please contact us if we can make this document more accessible: pdxscholar@pdx.edu. 
The Effects of Green Smoothie Consumption on

Blood Pressure and Health-Related Quality of Life:

A Randomized Controlled Trial

by

Emiko Maeda

A thesis submitted in partial fulfillment of the requirements for the degree of

Master of Science

in

Health Studies

Thesis Committee:

Gary Brodowicz, Chair

Jennifer Young

Claire Wheeler

Portland State University

2013 


\begin{abstract}
Chronic diseases are among the leading causes of death globally, and as much as $80 \%$ of these deaths are reported to be preventable with proper diet and lifestyle.

Although extensive research has demonstrated that the increased consumption of fruits and vegetables offers protective health effects from many chronic illnesses, populations in both developed and developing nations consistently fall short of the recommended intake of 5 or more servings a day. This study investigated the effects of daily consumption of Green Smoothies for 4 consecutive weeks on blood pressure and healthrelated quality of life. Green Smoothies are a blended drink consisting of fruit, leafy greens and water. The study was a randomized controlled trial with a final sample of 29 volunteer participants. Data were collected at baseline and post-intervention and included anthropometric and physiologic measures, as well as a nutrition survey. The treatment group demonstrated trends toward improvements in waist circumference $(p=$ 0.026), waist-to-hip ratio $(\mathrm{p}=0.05)$, and symptoms of burden linked to diet $(\mathrm{p}=0.04)$, small intestine $(p=0.04)$, large intestine $(p=0.05)$, and mineral needs $(p=0.04)$.

Despite the lack of statistically significant reductions in blood pressure, the trend toward improvements in waist circumference and waist-to-hip ratio are considered to be useful and informative of health risk. Thus, the results of this study provide preliminary support for the consumption of Green Smoothies as a possible primary prevention effort for chronic conditions. It may also help to reduce health risks or even reverse the effects of chronic conditions.
\end{abstract}




\section{Acknowledgements}

The basic concept of the research was developed with the help of my mother, Mitsuyo Hamaya, and completed with the guidance of my Graduate Advisor, Gary Brodowicz, Ph.D. I would also like to give special thanks to Miriam Wolf and Nathan Whitney who devoted their time and energy to this experimental study; Clyde Dent and Jennifer Young for assistance in data analysis and constructive feedback, respectively; Henry Cooney for being an objective third party to assist in maintenance of anonymity of my subjects and for reviewing numerical data for accuracy; and finally to my study participants, without whom this study would not have been possible. 


\section{Table of Contents}

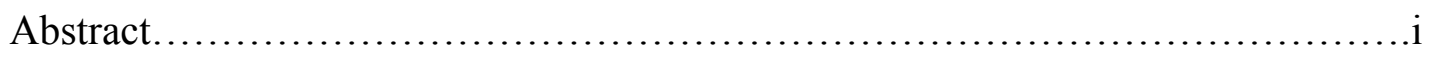

Acknowledgements....................................................ii

List of Tables...............................................................

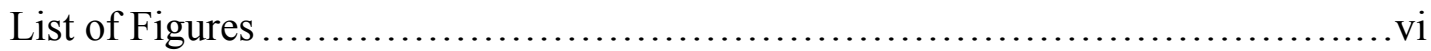

Glossary.........................................................viii

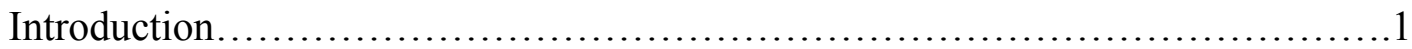

Significance...................................................

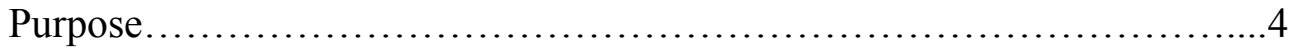

Research Questions...............................................4

Hypotheses..................................................4

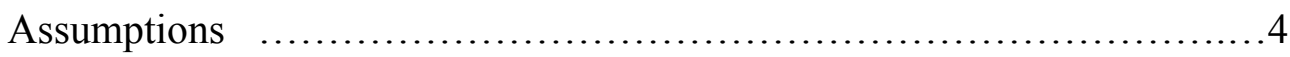

Review of Literature ...................................................6

Burden of Chronic Disease..........................................6

Inadequate Intake of Fruits and Vegetables............................

Benefits of Fruits and Vegetables...................................9

Benefits of Leafy Green Vegetables..................................11

Plant Fiber..................................................... 15

Phytochemicals..................................................17

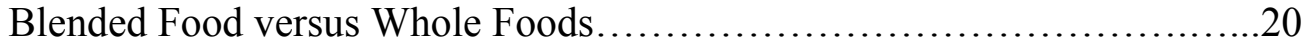

Blood Pressure and Chronic Disease.................................24

Blood Pressure and Fruit and Vegetable Intake.........................28

Health-Related Quality of Life..................................... 32

Methods.................................................................. 34

Overview....................................................... 34 
Subjects....................................................................

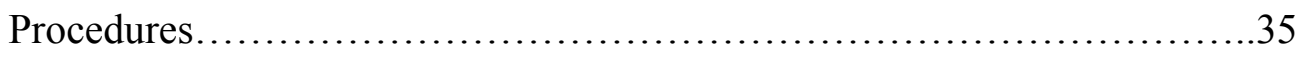

Materials \& Equipment.............................................. 38

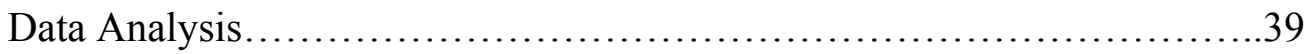

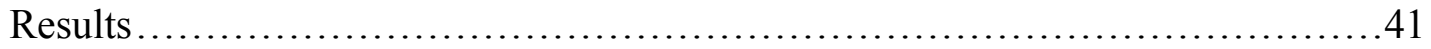

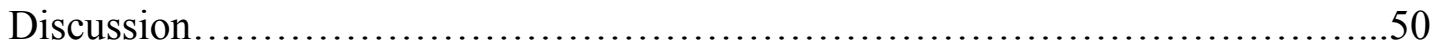

Blood Pressure .................................................... 50

Waist Circumference and Waist-to-Hip Ratio.............................52

Health-Related Quality of Life.......................................53

Nutritional Assessment Questionnaire..................................54

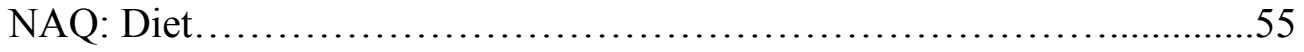

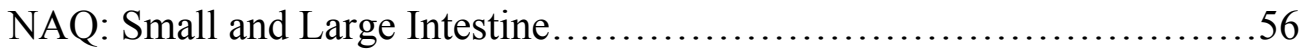

NAQ: Mineral Needs...............................................5

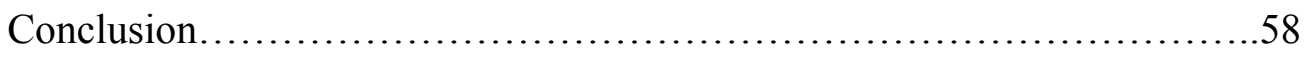

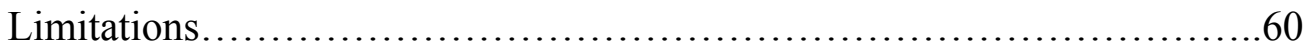

Directions for Future Research......................................63

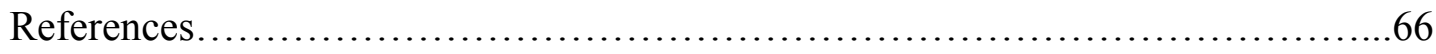

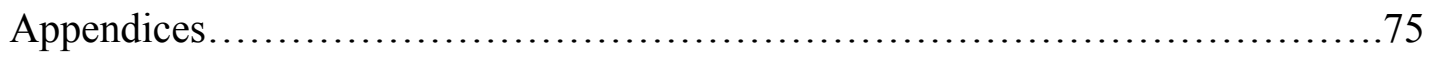

Appendix A: Informed Consent........................................ 75

Appendix B: Data Sheet..............................................

Appendix C: Nutritional Assessment Questionnaire........................79

Appendix D: Santa Ana Health Questionnaire ...........................83

Appendix E: Green Smoothie Schedule................................85

Appendix F: Green Smoothie Instructions...............................86

Appendix G: Flyer for Volunteer Recruitment........................... 87

Appendix H: NAQ Subsection Pre-Post Difference Scores..................88 


\section{List of Tables}

Table 1: Subject Characteristics at Baseline.................................42

Table 2: Mean Changes in Study Variables in Control and Treatment Groups......44 


\section{List of Figures}

Figure 1: Comparison of Control and Treatment Groups Waist Circumference

Pre-Post Difference Scores.........................................46

Figure 2: Comparison of Control and Treatment Groups Waist-to-Hip Ratio

Pre-Post Difference Scores......................................... 47

Figure 3: Comparison of Control and Treatment Groups NAQ Diet Subsection

Pre-Post Difference Scores......................................... 47

Figure 4: Comparison of Control and Treatment Groups for NAQ Small Intestine

Subsection Pre-Post Difference Scores................................48

Figure 5: Comparison of Control and Treatment Groups for NAQ Large Intestine

Subsection Pre-Post Difference Scores..............................48

Figure 6: Comparison of Control and Treatment Groups for NAQ Mineral Needs

Subsection Pre-Post Difference Scores................................49

Figure 7: Comparison of Control and Treatment Group Weight Pre-Post

Difference Scores...................................................88

Figure 8: Comparison of Control and Treatment Group Estimated Percent Fat

Pre-Post Difference Scores........................................89

Figure 9: Comparison of Control and Treatment Group Systolic Blood Pressure

Pre-Post Difference Scores..........................................90

Figure 10: Comparison of Control and Treatment Group Diastolic Blood Pressure

Pre-Post Difference Scores.........................................91

Figure 11: Comparison of Control and Treatment Group Resting Heart Rate

Pre-Post Difference Scores.......................................92

Figure 12: Comparison of Control and Treatment Group NAQ Lifestyle

Subsection Pre-Post Difference Scores...............................93

Figure 13: Comparison of Control and Treatment Group NAQ Upper GI

Subsection Pre-Post Difference Scores...........................94

Figure 14: Comparison of Control and Treatment Group NAQ Liver/Gallbladder

Subsection Pre-Post Difference Scores..............................95

Figure 15: Comparison of Control and Treatment Group NAQ Fatty Acids

Subsection Pre-Post Difference Scores............................96 
Figure 16: Comparison of Control and Treatment Group NAQ Sugar Handling Subsection Pre-Post Difference Scores...............................97

Figure 17: Comparison of Control and Treatment Group NAQ Vitamin Needs Subsection Pre-Post Difference Scores................................98

Figure 18: Comparison of Control and Treatment Group NAQ Adrenal Subsection Pre-Post Difference Scores...............................99

Figure 19: Comparison of Control and Treatment Group NAQ Pituitary Subsection Pre-Post Difference Scores............................100

Figure 20: Comparison of Control and Treatment Group NAQ Thyroid Subsection Pre-Post Difference Scores..............................101

Figure 21: Comparison of Control and Treatment Group NAQ Men Subsection Pre-Post Difference Scores..............................102

Figure 22: Comparison of Control and Treatment Group NAQ Women Subsection Pre-Post Difference Scores.............................103

Figure 23: Comparison of Control and Treatment Group NAQ Cardio Subsection Pre-Post Difference Scores.

Figure 24: Comparison of Control and Treatment Group NAQ Kidney Subsection Pre-Post Difference Scores.

Figure 25: Comparison of Control and Treatment Group NAQ Immune System Subsection Pre-Post Difference Scores. 


\section{Glossary of Terms}

Blood Pressure: Resting blood pressure is the pressure of circulating blood against the walls of the blood vessels inside the body. Hypertension (also known as high blood pressure) is a risk factor for cardiovascular disease, the leading cause of death in the United States (Hung et al., 2004)

Disability-Adjusted Life Year (DALY): Year of 'healthy' life lost. “... a measurement gap between current healthy status and an ideal health situation where the entire population lives to an advanced age, free of disease and disability." ("Metrics: Disability-Adjusted Life Year,”)

Green Smoothie: A blended beverage consisting of leafy green vegetables, water and fruit

Health-Related Quality of Life: A subjective, self-assessed measure that encompasses both physical and mental health

Nutritional Assessment Questionnaire: A 322-question health survey of daily habits, including — but not limited to—variables such as diet, health symptoms, sleep patterns, digestive function, emotional and psychological well-being, symptoms specific to gender, physical health, and energy level

Santa Ana Health Screen Questionnaire: Generic health survey used for the purpose of determining adequate health to qualify for participation in this study 
Skinfold Calipers: A device used to measure skinfold thickness, usually for the purpose of estimating body fat

Sphygmomanometer: The cuff used to measure blood pressure with the use of a stethoscope 


\section{Introduction}

In the last several decades, scientific exploration within the field of nutrition has provided an abundant amount of information pertaining to human health and wellness. Despite millennia of humans presumably feeding themselves nutritiously and adequately, many populations around the globe currently suffer from chronic and degenerative conditions at rates never before seen. With improved economic conditions and industrialized food, malnourishment and nutritional deficiencies in developing countries that have previously plagued populations have now been replaced with chronic conditions that had until recently predominantly existed in developed and wealthier nations (Nestle, 1999).

With the advent of agriculture and industrialization, populations are now steeped in an ample food supply with access to food unlike any other time in history. It seems predictable that with such an overabundance of food that many populations worldwide are not only becoming heavier, but also suffering from health conditions (e.g., cardiovascular disease, obesity, diabetes) precipitated by the physiological stresses of improper diet and excess body weight. Disseminating information about proper nutritional habits is warranted now more than ever, especially when considering that cardiovascular disease and cancer are the leading causes of death in America, and cardiovascular disease the leading cause of death in the world (Cottee, 1999; "Leading Causes of death,”; Nestle, 1999; “Top 10 causes of death,”, n.a.). Many chronic conditions (including — but not limited to—obesity, cancer, diabetes, hypertension, and CVD) may not only be prevented with proper nutrition, but their progression may also be 
slowed or reversed (Koertge et al., 2003; Ornish et al., 1990). It is therefore of utmost importance that people be informed about the dietary habits that will benefit their health and well-being.

Clinical and epidemiological research indicates that $80 \%$ or more of current chronic ailments and premature deaths are preventable with changes in diet and lifestyle (Anand et al., 2008; Mills, 2012). Research also indicates that increasing the consumption of fruits and vegetables offers a variety of beneficial health effects, preventing much of the damage of chronic ailments (Appel et al., 1997; Zhang et al., 2011). Public health research has demonstrated that not only are most Americans still deficient in their fruit and vegetable intake, but that a significant barrier - aside from the cost - to the consumption of fresh fruits and vegetables is incorporating them into their daily routine (Blatt, Roe, \& Rolls, 2011). Thus, more recent experimental studies have attempted to use liquid fruit and vegetables as soups or juices to both increase average fruit and vegetable consumption and enhance the ease of consumption.

The basis of this nutritional therapy study is grounded in the scientific understanding that increased consumption of fruits and vegetables offers many protective effects on human health. Extensive nutritional and public health studies have demonstrated that adequate consumption of fruits and vegetables has preventative effects on cardiovascular disease, cancers, hypertension, and other chronic conditions such as diabetes and obesity (Bazzano et al., 2002; Hung et al., 2004; Lampe, 1999; Roberts \& Barnard, 2005). Extensive public health efforts have also been made to increase proper fruit and vegetable consumption by initiating programs such as the 'Five a Day for Better 
Health', 'Gimme 5', the Healthy People campaign, and the emergence of organizations such as the 'Produce for Better Health Foundation' and Michelle Obama's 'Let's Move' campaign that focuses attention on proper diet and nutrition. Furthermore, this study aims not only to reduce barriers to fruit and vegetable consumption by simplifying inclusion into the diet by making one blended beverage a day, but also to demonstrate the benefits of blended vegetables versus those in whole and cooked form.

\section{Significance}

Epidemiological data indicate that the majority of developed populations are deficient in fruit and vegetable consumption, which has been shown to have protective health effects on chronic conditions such as hypertension, cancer, stroke, heart disease and diabetes (John, Ziebland, Yudkin, Roe, Neil, 2002; Kiefer et al., 2004; Roberts \& Barnard, 2005). To date, there is limited research on nutritional therapy methods utilizing blended fruit and vegetable beverages to increase fruit and vegetable consumption. This study adds to the body of research on nutritional therapy practices using a simple method for increasing fruit and vegetable consumption. Implementation of strategies to increase fruit and vegetable consumption on both individual and population levels may be effective in reducing the prevalence of chronic disease and premature death.

Review of pertinent literature suggests this study is the first of its kind to investigate the possible health effects resulting from daily consumption of a self-made beverage made of fruit and vegetables. This study not only attempts to improve consumption of fruits and vegetables but also explores the possibility of utilizing Green 
Smoothies as a preventive measure against chronic disease and to improve health-related quality of life.

\section{Purpose}

The purpose of this study was to investigate the effects of daily Green Smoothie consumption for 4 consecutive weeks on resting blood pressure and health-related quality of life.

\section{Research Questions}

- Does a 4-week period of daily Green Smoothie consumption reduce resting blood pressure?

- Does a 4-week period of daily Green Smoothie consumption reduce symptoms of burden (i.e., improve health-related quality of life)?

\section{Hypotheses}

- $\mathrm{H}_{1}$ : Four weeks of daily Green Smoothie consumption will significantly reduce resting blood pressure (systolic and/or diastolic).

- $\mathrm{H}_{2}$ : Four weeks of daily Green Smoothie consumption will significantly reduce symptoms of burden (i.e., improve health-related quality of life).

\section{Assumptions}

This research makes certain assumptions regarding the efficacy of method of outcome measures and the honesty and compliance from subjects. It is assumed that blood pressure measurements made during data collection sessions are a valid indication 
of average resting blood pressure. It is also assumed that all subjects answered the Nutritional Assessment Questionnaire (NAQ) honestly and followed the Green Smoothie consumption protocol as instructed. Finally, it is assumed that all participants followed instructions to refrain from changing their daily exercise and eating habits for the duration of the study. 


\section{Review of Literature}

\section{Burden of Chronic Disease}

Chronic diseases (i.e., cardiovascular disease, cancer, diabetes, and stroke) are the leading causes of death and disability globally, and their incidence of burden continues to rise at an alarming rate throughout the world (Broekmans et al., 2001; Lock, Pomerleau, Causer, Altmann, \& McKee, 2005; “Top 10”). According to the Centers for Disease Control and Prevention (CDC), chronic disease accounts for more than $70 \%$ of deaths each year and 1 in every 2 adults is afflicted with at least one chronic illness ("Chronic Disease and Health Promotion"). Furthermore, 21\% of the US population suffered from multiple chronic conditions in 2000 (Anderson \& Hovarth, 2004). The CDC reports that chronic diseases are not only the most costly of health problems but also the most preventable of conditions plaguing the US population. Chronic disease is one of the main contributors to the burden of disability that not only reduces the quality of life but also increases the need for more social services and reduces overall productivity, resulting in costly economic losses (Klijs, Nusselder, Looman, \& Mackenback, 2011). For both developed and developing countries, the increased stress of the rapidly climbing incidence of chronic illness further cripples nations' abilities to provide care for their ailing populations, and effectively undermines their efforts to increase life expectancy and spur economic growth ("WMA statement”).

In light of the daunting reality of our current ailing populations, policy makers and health officials are making it their primary concern to put forth major efforts to promote habits and lifestyle changes that prevent these debilitating chronic illnesses from 
developing in the first place. Considering the price of expensive tertiary care methods such as surgery and chemotherapy, prevention is likely to be a more effective and cost efficient method of addressing this health crisis (Partnership for Prevention, 2009). In addition, dietary changes and modifications in physical activity can reduce many symptoms and side effects of these chronic conditions and may even reverse the progression of some illnesses (Anderson \& Hovarth, 2004).

\section{Inadequate Intake of Fruits and Vegetables}

Decades of epidemiologic and clinical research have demonstrated the multitude of benefits of fruits and vegetables in the human diet for protection from acute and chronic illness (Lock, Pomerlau, Causer, \& McKee, 2004; Lock et al., 2005). For the past 20 years, the United States Department of Agriculture (USDA) has recommended at least 5 servings of fruits and vegetables in the daily diet for the US population (Shenoy et al., 2010). However, intake of fruit and vegetables consistently falls below recommended levels for both school-aged children and adults. A study conducted by the Center for Nutrition Policy and Promotion, a sector within the USDA, used data from the 1999-2000 National Health and Nutrition Examination Survey to investigate fruit and vegetable consumption in comparison to the USDA recommendation. The data from over 8000 respondents showed that only $40 \%$ of Americans consumed 5 or more servings of fruit and vegetables daily (Guenther, Dodd, Reedy, \& Krebs-Smith, 2012). With primary prevention of chronic illness being an important approach to the currently devastating burden of chronic illness, falling short of efforts to increase fruit and vegetable 
consumption should be a primary concern for policymakers and healthcare officials (Lock et al., 2005).

Although it is important to consider barriers to fruit and vegetable consumption such as cost and access for certain populations, inadequate consumption of fruits and vegetables is common for populations without issues of resource ("Preventing chronic disease"). Until recently, aside from USDA recommendations, public efforts to enhance the consumption of fruits and vegetables had remained solely within the educational system spanning only through high school. Healthy People, a campaign spearheaded by the Centers for Disease Control and Prevention, is a framework for the nation's health priorities with a focus on disease prevention through efforts such as increasing physical activity and increasing fruit and vegetable consumption ("Healthy People"). In acknowledgement of the history of consistently inadequate intake, Healthy People 2010's goals remained realistic and strived to increase the populations' fruit consumption to 2 or more servings of fruit a day for $75 \%$ of the population, and a goal of 3 or more servings of vegetables for $50 \%$ of the population ("State Indicator Report"). These goals were based on data compiled in 2009 that documented fruit and vegetable intake from both adults and adolescents. Fruit intake for adolescents and adults was almost equal with $32.2 \%$ and $32.8 \%$ consuming 2 or more serving of fruit daily, respectively. Adults appeared to be much better about consuming vegetables, with data indicating that adults and adolescents consumed 3 or more servings of vegetables a day at percentages of $27.4 \%$ and $13.2 \%$, respectively. However, both adults and adolescents appeared deficient overall when examining the consumption of 2 or more servings of fruit and 3 or more 
servings of vegetables each day, with a prevalence of $14.0 \%$ for adults and $9.5 \%$ for adolescents.

A major strength in the efforts of the CDC to address inadequate fruit and vegetable consumption is their focus on addressing issues of access and cost of fruits and vegetables and policies favoring the promotion of healthy foods. However, fruit and vegetable intake remains below recommended amounts for populations without issues of access, thus indicating that increased efforts must be made to boost the consumption of these beneficial foods in the prevention of chronic diseases and protection of health.

\section{Benefits of Fruits and Vegetables}

Increasing consumption of fruits and vegetables in the daily diet has been a focus in the improvement of health and prevention of many chronic and degenerative diseases for several decades and is a method that is increasingly encouraged by public health efforts and by medical practitioners. To date, cardiovascular disease and cancers are the two leading causes of death in industrialized countries and it is estimated that one third of cancer deaths can be prevented through dietary modification (Liu, 2000).

The prevention of debilitating or life-threatening conditions through diet modification is not only a solution easily addressed by making thoughtful decisions at mealtimes and making changes to other daily habits, but is also significantly more costeffective than expensive and potentially dangerous tertiary methods (e.g., chemotherapy and surgery) (Ornish, 2008; Taylor, Hampl, \& Johnston, 2000). In 2003, RAND initiated a study investigating the possible benefits of a unified medical records system that would ease the process of linking patients with providers, and better track the medical history of 
patients. They discovered that the system would enhance the capability to focus on preventative efforts, and projected that prevention and disease management could result in a total savings of almost $\$ 81$ billion in annual health expenditure (Taylor et al., 2005).

The beneficial role of fruits and vegetables, with their high fiber content and abundance of vitamins and minerals, has been widely researched in the last several decades. They have been accepted as having beneficial effects on human health, initiating public health efforts such as the ' 5 a Day for Better Health', the DASH diet, and inclusion in the American Heart Association (AHA) dietary guidelines to increase consumption to improve human health (Tucker, 2004). The great majority of epidemiological studies as well as clinical trials and in-vitro studies demonstrate improved health and reduced risks for a variety of chronic conditions such as heart disease, cancer, and diabetes, are lower for populations with a higher intake of fruits and vegetables (Chu, Sun, Wu, \& Liu 2002; Liu, 2003; Rowland,1999; Schreiner \& Huyskens-Keil, 2006; Steinmetz and Potter, 1996).

Existing research, however, demonstrates that the health benefits of fruits and vegetables go far beyond their low energy density, fiber content, and richness in vitamins and minerals. Beyond their nutritive value, studies have shown that increased fruit and vegetable intake assists in the prevention of chronic and degenerative diseases, thereby earning fruits and vegetables the moniker of 'functional foods'(i.e., foods that contain physiologically active compounds that provide health benefits beyond the basics of nutrition) (Whitney \& Rolfes, 2008). 
Although there is much clinical and epidemiological evidence demonstrating the protective role of plant-based foods, it is difficult to pinpoint the exact mechanisms through which they confer their benefits to human health. Plant-based foods have a variety of independently beneficial attributes such as high fiber content, phytochemicals, antioxidants, unsaturated fats, plant protein, vitamins, minerals, flavenols, and high water content; these likely have a synergistic effect that mediate their health protective qualities (Cottee,1999; Hu, 2003). Clinical studies that attempt to find the mechanisms responsible for the health benefits of fruits and vegetables by isolating specific vitamins and minerals often result in inconsistent data, rendering many studies on the topic inconclusive and needing 'further investigation'. Whether the process of extraction, the concentration of the element investigated, or the lack of fiber inherent in the whole food, studies on single nutrient approaches appear reductive and more current research recommends incorporating the whole foods into the diet for the most benefit (Broadhurst, 1997; Chun et al., 2005; Schreiner \& Huyskens-Keil, 2006; Tucker, 2004).

\section{Benefits of Leafy Green Vegetables}

Decades of research has been devoted to the benefits of fruits and vegetables on human health and has promulgated broad public health efforts to promote their inclusion in the diet for better health. Efforts such as the ' 5 a Day for Better Health', Healthy People, and strong recommendations from the government have played a role in promoting increased consumption of these beneficial foods. Luckily, surveys show that these efforts have not been in vain and that fruit and vegetable consumption has indeed increased since these public efforts were put in place. According to national surveys, 
consumption of fruits and vegetables increased 12\% between 1991 and 1994, and the average American 25-75 years of age consumed an average of 5.2 serving of fruits and vegetables per day during this time (Johnston, Taylor, \& Hampl, 2000; Taylor et al., 2000). Between 1994 and 1996, 45\% of Americans were getting their five or more servings of fruits and vegetables, a 13\% increase from surveys in earlier years (Johnston et al., 2000).

However, food intake questionnaires demonstrate that nearly $30 \%$ of the fruits and vegetables consumed were bananas, iceberg lettuce, orange juice and French Fries (Johnston et al., 2000). Tomato products and dark green vegetable consumption remained low at 0.5 and 0.2 servings a day, respectively, revealing that Americans remain deficient in consuming the fruits and vegetables that confer the most beneficial health effects (Johnston et al., 2000).

Only more recently have leafy green vegetables begun to be examined apart from fruits, and also investigated independently of other vegetables. Although few in number, studies have reported significant findings on the protective effects of leafy green and cruciferous vegetables, demonstrating that their regular inclusion in the human diet may be superior to fruits and other less nutritive vegetables (Hung et al., 2004; Johnston et al., 2000; Zhang et al., 2011). Despite the benefits of fruits and vegetables categorically, research consistently indicates that certain fruits and vegetables such as tomatoes, citrus fruits and dark green and cruciferous vegetables are particularly associated with reduced risk of cancer (Hung et al., 2004; Johnston et al., 2000; Zhang et al., 2011). 
Aside from the these micronutrients, other substances in dark green vegetables, the phytochemicals, are biologically active in humans and may mitigate diseases and promote health (Hasler, 1998). The biological activities of the phytochemicals are diverse, but the major functional effects may be grouped as antioxidant, carcinogen detoxifier or cell regulator (Hasler \& Blumberg, 1999)... Interestingly, the items with the highest ORAC (oxygen radical absorbance capacity) or QR (quinone reductase) induction score, dark green and cruciferous vegetables, onions and citrus fruits, are the fruits and vegetables most commonly related to reduced cancer risk in epidemiological investigations (Ferguson, 1999 as cited in Johnston et al., 2000, p. 3066).

A large prospective, population-based cohort study in China involving over

134,000 Chinese adults examined the effects of cruciferous vegetables, non-cruciferous vegetables, all vegetables and total fruit consumption on the risk of cause-specific and all-cause mortality. Using data from the Shanghai Women's Health Study and Shanghai Men's Health Study, the researchers found an inverse relationship between overall fruit and vegetable intake and risk of total mortality for both genders, and a dose-dependent pattern for cruciferous vegetables (Zhang et al., 2011). Although cancer mortality was not shown to be affected by increased intake of these foods, cardiovascular disease risk was reduced, especially with increased consumption of cruciferous vegetables. Zhang et al. (2011) commented:

A unique feature of cruciferous vegetables is their high content of sulfurcontaining compounds, known as glucosinolates. It has been suggested that some breakdown products of glucosinolates may confer protection against certain cancers by modulating the activity of enzymes involved in detoxifying carcinogens and metabolizing sex hormones... One particular glucosinolate breakdown product, sulforaphane, has been shown to reduce oxidative stress and inflammation in the cardiovascular system by activating transcription factor $\mathrm{Nrf2}$ (p. 245).

Hung et al. (2004) performed another large prospective cohort study that collected data from the Nurses' Health Study and the Health Professionals' Follow-up Study 
consisting of over 173,000 participants. At the outset of the study, all participants were free of major chronic diseases and baseline measures included a basic medical history and food frequency questionnaires. Dietary information was collected every four years, and medical occurrences such as — but not limited to - strokes, cancers, and heart attacks, were updated every two years.

Resulting data demonstrated that the relative risk for major disease was 0.95 for subjects within the highest quintile of fruit and vegetable consumption and that total fruit and vegetable intake had an inverse association with cardiovascular disease (Hung et al., 2004). However, food group analysis exhibited that leafy green vegetable intake had the strongest inverse association with major chronic diseases and cardiovascular diseases, with just one serving of leafy greens per day showing a relative risk of 0.95 and 0.89 for major chronic diseases and cardiovascular disease, respectively (Hung et al., 2004). Furthermore, the team observed an inverse relationship between cruciferous vegetable intake and total cancer incidence for male current smokers and non-vitamin users but not for women. Also, an inverse association was noted between cruciferous vegetable intake and bladder cancer in men.

Hung et al. (2004) concluded that their findings suggest a high consumption of fruits and vegetables - particularly green leafy vegetables — is associated with reductions in risk for chronic diseases, and primarily cardiovascular diseases. Although associations with reduction in overall cancer risk were not found to be linked with fruit and vegetable consumption in this study, extensive epidemiological and experimental studies support 
protective effects from certain types of cancers (Feskanich, 2000; Key et al., 2004; Terry et al., 2001).

Despite impressive improvements in Americans' consumption of fruits and vegetables in the last decade, it appears that the inclusion of the most health giving fruits and vegetables, namely citrus fruits and dark green and cruciferous vegetables, remains inadequately supplied in the diet (Johnston et al., 2000). With experimental and epidemiological research indicating the robust benefits of these foods and their role in reductions of chronic disease risk, it is of paramount importance that health officials and professionals take a stronger stance in promoting their inclusion in the daily diet.

\section{Plant Fiber}

The true benefit of dietary fiber was first discovered in 1977 when Denis Burkitt and Hugh Trowell linked the low incidence of chronic diseases such as diabetes, cancer and coronary heart disease in African populations with high intakes of unrefined plant foods (Liu, 2003). This discovery led to the 'fiber hypothesis' that postulated that increased intakes of fiber rich plant foods would confer health benefits protective against the chronic diseases that had begun to plague the Western world. The connection between high fiber diets and reduced risk of chronic disease has since become a cornerstone of dietary wisdom and continues today to be a leading approach for preventing chronic illness. Clinical and epidemiological studies have increasingly demonstrated the benefits of fiber in the control and prevention of hypertension, obesity, cardiovascular disease, glycemic control, high cholesterol and intestinal disorders (Anderson, Smith, \& Gustafson, 1994; Nishida, Uauy, Kumanyika, \& Setty, 2004). 
The fiber of plant-based foods is a beneficial characteristic of plant foods that has received extensive attention in nutritional science and many of its benefits have been documented, especially its role in controlling serum lipids and lowering total and lowdensity lipoprotein (LDL) cholesterol (Theuwissen \& Mensink, 2008). The theoretical basis for the benefits of plant fiber is the minimization of hepatic cholesterol synthesis as well as enhanced excretion of cholesterol in the feces through binding of bile acids in the small intestine (Anderson et al., 2009). Furthermore, high fiber foods increase intestinal motility, reduce risks of gastrointestinal disorders, slow digestion of food and provide a lasting feeling of fullness, promote regularity and help control insulin levels (Anderson et al., 1994). Compared with non-vegetarians, vegetarians consistently show reduced rates of heart disease, cancer, hypertension, high cholesterol and obesity, presumably as a partial result of their higher dietary fiber content (Appel et al., 2006; Fraser, 2009; Sticher, Smith, \& Davidson, 2010). However, the basis for these effects is difficult to ascertain because dietary fiber content of the diet is often coincident with other dietary components such as saturated and unsaturated fats, minerals, and antioxidants (Anderson et al., 1994).

Fiber consumption in the US is currently less than half of what is recommended for good health (Anderson et al., 2009; Clemens et al., 2012) and current studies supporting the benefits of dietary fiber urge a doubling or more of current fiber intakes for Americans for the prevention of chronic disease (Clemens et al., 2012; Lupton, \& Turner, 2003; Theuwissen \& Mensink, 2008). Unlike other micro and macronutrients, there is no recommended upper limit for dietary fiber, with the only adverse effect of overconsumption being some intestinal discomfort. The World Health Organization 
(WHO) and the Food and Agriculture Organization (FAO) of the United Nations currently encourage the consumption of recommended amounts of fruits and vegetables and whole grain foods to meet necessary fiber requirements in order to gain the health protective benefits of dietary fiber (Nishida et al., 2004).

\section{Phytochemicals}

By definition, phytochemicals are plant-derived bioactive compounds that have been linked to reduced risks for major chronic diseases (Liu, 2003). Phytochemicals are responsible for providing fruits and vegetables their defining characteristics of vibrant color, aroma, and spice. Their physiological benefits include antioxidant mechanisms, ability to mimic hormones, and stimulate enzymes within the body (Tucker, 2004). The principal feature putting phytochemicals at the forefront of preventative health is their ability to reduce free radical oxidative stress. Oxidative stress - an unavoidable byproduct of an aerobic life - is an imbalance between reactive oxygen species produced in the body and the body's ability to quench their potentially toxic effects (Storz \& Imlay, 1999). Oxidative stress caused by the overabundance of free radicals, a particularly damaging species amongst reactive oxygen species, is significantly harmful and can cause serious damage to DNA, lipids and proteins, resulting in increased risks for chronic conditions such as cancer and cardiovascular disease (Chu et al., 2002; Wang, Cao, \& Prior, 1996). With oxidative stress lying at the core of many current chronic conditions that plague industrialized populations, the importance of choosing foods replete with antioxidant compounds and maintaining a balance is essential in the promotion of good health and protecting cellular systems from damage (Liu, 2003). The abundance of 
antioxidants assists in the prevention of tissue damage caused by free radicals by promoting decomposition of free radicals, scavenging and destroying them, or inhibiting their formation (Young \& Woodside, 2001).

Phytochemicals possess other protective health benefits such as reducing platelet aggregation, modulating cholesterol synthesis and absorption, reducing blood pressure (systolic and/or diastolic), inhibiting LDL oxidation, supporting immune function, reducing inflammation, providing anti-fungal effects, regulating blood sugar and acting as an anti-carcinogenic (Broadhurst, 1997; Heber, 2004; Schreiner \& Huyskens-Keil, 2006). However, despite clinical evidence demonstrating these protective effects, identifying the exact phytochemicals responsible and their effective dosage has been a challenge to researchers.

Currently, over 8,000 phytochemicals have been identified, but many remain yet unidentified (Liu, 2003). In general, phytochemicals can be categorized under three main subgroups: flavonoids, carotenoids, and alkaloids. Although their mechanisms of action are not completely clear and often overlap, flavonoids typically inhibit platelet aggregation and adhesion, inhibit LDL oxidation, are anti-inflammatory, and are generally responsible for the antioxidant characteristics of phytochemicals. Carotenoids are mostly responsible for lowering cholesterol and providing protection from cancer, and alkaloids are anti-carcinogenic (Liu, 2003).

Studying the beneficial effects of phytochemicals is not without significant challenges. Different plants contain varying phytochemicals with different structures that result in distinct beneficial mechanisms at varying levels. Furthermore, varied absorption 
throughout the intestinal tract for each phytochemical and inter-individual variability in their metabolism further complicates measurement and distinction of the specific effects on health (Chu et al., 2002).

Consensus among researchers investigating the role of phytochemicals thus far is that phytochemicals have an additive and synergistic effect in which the natural combination of phytochemicals within foods appears to optimize their antioxidant and health promoting qualities (Broadhurst, 1997; Chun et al., 2005; Schreiner \& HuyskensKeil, 2006; Tucker, 2004). Clinical efforts to streamline health benefits and find a 'magic bullet' cure by isolating specific phytochemicals and utilizing them as supplements in human clinical trials have been disappointing. Many studies involving isolated phytochemicals often result in non-significant results or have adverse effects on study subjects (Broadhurst, 1997; Chu et al., 2002; Chun et al., 2005; Liu 2003).

Reviews of the literature on phytochemicals consistently advocate for the inclusion of more fruits and vegetables in the diet in lieu of expensive supplements and extracts, which have the potential to do more harm than good (Liu, 2003). Unlike supplements, the levels of phytochemicals found in whole foods are far below the levels of toxicity found to have adverse effects in clinical trials, thus further supporting their inclusion into daily diets. In her article discussing the importance of phytochemicals in the protection from heart disease, Tucker (2004) concludes:

It is becoming increasingly clear that single-nutrient approaches are not sufficient. Rather, the total dietary pattern needs to be attended to, with an emphasis on use of whole, minimally processed foods so that the full complement of naturally occurring phytochemicals and nutrients are consumed along with the natural fiber and selected fatty acids. (p. 292). 


\section{Blended Foods versus Whole Foods}

When comparing the benefits of whole foods and blended foods it is important to consider making the comparison on two separate levels: first, on a nutritional level (i.e., when examining the bioavailability of the nutrients within the foods), and second, on a consumer level (i.e., the ease and ability to incorporate the foods into one's daily diet). Although clinical research on the benefits of blended or liquefied foods is still limited, several studies have attempted nutritional interventions using alternative forms of fruit and vegetable administration aside from the traditional methods of urging the consumption of whole fruit and vegetables to study subjects. To date, studies have used vegetable juices, fruit juices, soups, and fruit and vegetable concentrates in the form of capsules as alternative methods of administration.

Shenoy et al. (2010) conducted a randomized controlled study to investigate the possibility of using a commercial vegetable juice as a practical way to meet USDA dietary guidelines for vegetable intake, and to assess the effects of the juice on cardiovascular health. The three-arm trial had participants drink 0,8 , or 16 ounces of Campbell's V8 juice each day for 12 weeks as well as receive education on the Dietary Approaches to Stop Hypertension (DASH) diet. At baseline, all groups had intakes of vegetables lower than the USDA recommended servings. After the 12-week trial, results indicated that for subjects consuming the juice, daily vegetable consumption significantly increased to 4.3 and 6.5 servings for the 8-ounce/day and 16-ounce/day groups, respectively. Furthermore, participants in the juice groups considered to be 'prehypertensive' at the beginning of the study exhibited significant reductions in blood 
pressure by the end of the trial. Although the researchers can only speculate how much of these reductions were attributable to the juice consumption and not the DASH diet, the success of utilizing the blended vegetables to increase overall vegetable consumption cannot be overlooked.

Based on their findings, the researchers concluded that the incorporation of 1-2 cups of vegetable juice in the diet was an 'effective and acceptable way for healthy adults to close the dietary vegetable gap' and that an increase of vegetable intake was linked with a reduction in blood pressure for subjects who were prehypertensive at the beginning of the trial (Shenoy et al., 2010, p. 9). The researchers also noted that dietary training alone was insufficient to elicit any significant change in vegetable intake and acknowledged the need for dietary behavior change programs that go beyond increasing personal awareness of the benefits of increased consumption of vegetables (Casagrande, Wang, Anderson, \& Gary, 2007; Guenther et al., 2012; Shenoy et al., 2010).

The taste of vegetables is also a barrier that limits daily vegetable intake for many individuals. One study (Blatt et al., 2011) investigated the effects of implementing pureed vegetable soups in place of meals once a week for 3 weeks. The aim of the study was to examine whether the incorporation of the pureed vegetables could increase daily vegetable intake, reduce energy intake at each meal, and decrease overall energy intake. Researchers covertly replaced high energy density ingredients in the 'meals' with large amounts of vegetables without any observed change in subjects' feelings of hunger or satiety. The results of this crossover trial showed that the addition of pureed vegetable soups not only reduced overall energy intake but also was an effective method of 
increasing vegetable consumption (Blatt et al., 2011). The bitter taste of vegetables was concealed by pureeing them into a soup, and subjects were both satisfied and satiated with the liquid meal.

Both the US Department of Health and the CDC have previously recommended substituting vegetables in place of higher density foods at meal times to reduce overall energy intake. However, despite these recommendations, consumption of these health protective foods consistently fall short of recommended levels. Although many studies have attempted to increase vegetable intake by substituting vegetables for meats and grains, or providing vegetables as a side dish, few have investigated the process of incorporating vegetables by liquefying or pureeing the vegetables (Blatt et al., 2011).

Blended and liquefied foods offer increased ease of consumption and are easier to introduce into a daily routine; furthermore, they may alter the bioavailability of the nutrients within their ingredients. A study from the Center for Human Nutrition at UCLA investigated the bioavailability and antioxidant effects of orange juice on a small sample of men and women (Franke, Cooney, Henning \& Custer, 2005). The researchers concluded that after the 3-week trial, not only did the orange juice consumption offset the daily intake of other snacks and result in smaller portion sizes of meals when the orange juice was consumed throughout the day, but that even in as short of an intervention as 3 weeks, orange juice was:

[an]'excellent food source to increase blood levels of a series of valuable phytochemicals... exert[ing] their activity by various mechanisms including a decrease in oxidative damage to select biological targets and thereby protect 
against chronic disorders especially, in at-risk populations with low antioxidant status' (Franke et al., 2005, p. 8).

Another study investigated the bioavailability of carotenoids in plant foods (Dutta, Chaudhuri, \& Chakraborty, 2005). The absorption of carotenoids poses multiple challenges due to their intracellular location and the tightness of their cellular matrix within the plant food, thus often limiting their uptake by the human body (Dutta et al., 2005). Studies have also shown that absorption of carotenoids from fruits and vegetables is better facilitated when consumed with fat (Brown et al., 2004; Tang, Dolnikowski, Russel, \& Grusak, 2005) and also after processing (e.g., pureeing or cooking) compared with consuming them raw or whole (Dutta et al., 2005). In a study investigating the effects of the vitamin A content of pureed spinach and carrots, Tang et al. (2005) found that the bioavailability of vitamin A was enhanced when spinach was consumed minced versus whole leaf (6.4\% vs. $5.1 \%$ ), thus supporting the need to 'process' the foods for increased absorption.

Further adding to the challenge of examining the effects of carotenoid absorption is their short retention time within a food product. Although processing such as cooking, blanching or blending may increase bioavailability, the length of processing time and method of processing greatly impacts the antioxidant value of plant foods through enzymatic and non-enzymatic oxidation (Szeto, Tomlinson, \& Benzie, 2002). Freezing, drying, peeling and juicing plant foods can lead to considerable reductions of carotenoids and lead to carotenoid destruction within plant foods, thus increasing their bioavailability, but making them considerably less potent (Dutta et al., 2005). Although differences in absorption are not large, it is important to consider how the slight but many variations 
affecting the bioavailability and nutritional content of plant foods depend on storage methods and preparation approaches. Judging from previous studies examining the benefits and drawbacks of quantifying the nutritional value of plant foods, it is clear that optimal methods of measuring the health protective qualities of plant foods and their nutrients continues to pose considerable challenges.

A research team at the Institute of Social Medicine in Vienna conducted a supplementation trial using dehydrated fruit and vegetable concentrates in capsule form to investigate serum antioxidant and folate levels in a pool of healthy adults (Kiefer et al., 2004). In this randomized crossover design study, 59 subjects were placed in either the control or treatment group for a span of 7 weeks before crossing over to the other group. Data were collected at baseline, 7 weeks, and 14 weeks, and results indicated a significant correlation between supplementation and increases in blood levels of vitamin $\mathrm{C}$, beta carotene, vitamin E, selenium and folate. The researchers concluded that supplementation such as the kind utilized in this study could be useful and beneficial in the protection of human health (Kiefer et al., 2004).

\section{Blood Pressure and Chronic Disease}

Elevated blood pressure, otherwise known as hypertension, is one of the major risk factors for cardiovascular disease. Cardiovascular disease (CVD) is any disease involving the heart or blood vessels and includes conditions such as ischemic heart disease, stroke, atherosclerosis, coronary artery disease, cerebrovascular disease, myocardial infarction, angina and aneurysms ("What is Heart Disease?" 
Cardiovascular disease accounts for the majority of deaths each year both nationally and globally, and is the number one cause of death for women ("Heart Disease."). Elevated blood pressure is the leading cause of CVD, which contributes to more than $66 \%$ of US deaths annually (Ferdinand et al., 2012). According to the World Health Organization, cardiovascular diseases are the leading cause of death and disability globally, taking more lives than any other cause ("Cardiovascular Disease,", n.a). According to the World Health Organization fact sheet on Cardiovascular disease (2012), in 2008 more than $30 \%$ of deaths worldwide were attributable to CVDs, and $13 \%$ of all deaths globally were attributable to elevated blood pressure alone Lawes et al. (2004). A meta-analysis of over 230 surveys that included the data of over 650,000 participants concluded that elevated blood pressure, namely elevated systolic blood pressure, was responsible for:

- $49 \%$ of ischemic heart disease, translating to almost 3 million deaths and 28.2 million disability-adjusted life years (DALYs) in the year 2000

- $62 \%$ of strokes, translating to 3.1 million deaths and 27.8 million DALY

- $76 \%$ of hypertensive diseases, contributing to 663,000 deaths and 5.4 million DALY in the year 2000

- $14 \%$ of other cardiovascular diseases, translating to 338,000 deaths and 2.8 million DALY (Lawes et al., 2004)

Cardiovascular disease is crippling populations in both developed and developing countries, and contributing to an enormous amount of burden on individuals and health services. The results of the meta-analysis indicate that "a considerable proportion of 
cardiovascular disease is related to non-optimal blood pressure, and this translates into an important portion of deaths and years of life lost to deaths and disability worldwide" (Lawes et al., 2004, p. 283). Cardiovascular disease currently accounts for almost 20\% of health care costs and $30 \%$ of Medicare expenditures, and productivity losses and related medical costs amount to nearly 450 billion dollars annually, with projections of tripling in the next 20 years (Ferdinand et al., 2012).

Although pharmacological methods are available and effective at controlling blood pressure, changes in lifestyle habits such as smoking cessation, improved diet and increased physical activity can not only reduce reliance on costly pharmaceuticals, but also offer other benefits to health. Thus, the control of blood pressure and prevention of developing hypertension is an important step in reducing cardiovascular mortality and morbidity.

Even small changes in blood pressure can have a significant impact on health risk on a population level. An incremental reduction of $3 \mathrm{mmHg}$ in systolic blood pressure may result in an $8 \%$ decrease in stroke mortality risk and a 5\% reduction in risk from coronary heart disease (Appel et al., 2006). Experimental studies have shown that reducing body weight by as little as 3-9\% is as effective for controlling blood pressure as reducing sodium intake, which has been a main focus contributing to efforts such as the DASH diet and health practitioner recommendations to reduce salt intake (Hermansen, 2000). However, a recent meta-analysis on dietary factors related to blood pressure indicated that inadequate mineral intake may be more influential than sodium intake for lowering blood pressure (Hermansen, 2000). 
In a long-term 32 year follow-up study of a homogenous population of over 3000 male executives, elevated blood pressure was found to increase mortality risk linearly in a systolic blood pressure range of 131-140 $\mathrm{mmHg}$ and a diastolic blood pressure range of 81-85 mmHg (Strandberg, Salomaa, Vanhanen, \& Pitkala, 2001). However, it is important to note that the data indicated that elevated blood pressure appeared to increase other risk factors, making it difficult to determine whether mortality risk increased due to the concurrent presence of other risk factors or if elevated blood pressure alone was responsible.

Efforts to reduce the incidence of elevated blood pressure will not only curtail the suffering and mortality of individuals, but also effectively help reduce debilitating national health care costs. It is estimated that reducing average population sodium intake to $2300 \mathrm{mg} / \mathrm{d}$ could save $\$ 18$ billion in health care costs annually, and a 5\% reduction in the prevalence of hypertension would save \$25 billion in 5 years (Ferdinand et al., 2012).

Extensive research indicates that elevated blood pressure is associated with risk of stroke and ischemic heart disease for both men and women, and that control of blood pressure is an effective method of reducing these health risks (Appel, 1999; Cakir \& Pinar, 2006; Hall, 2008). Even with a long history of heart disease, such conditions are not only treatable but often reversible through changes in lifestyle such as stress reduction and improved nutrition (Koertge et al., 2003; Ornish et al., 1990). The main behavioral risk factors for cardiovascular disease are unhealthy diet, physical inactivity, tobacco use and alcohol consumption ("Noncommunicable Diseases"). Due to the prevalence and danger of elevated blood pressure, practical means to prevent or control it 
are necessary. Changes in dietary habits, such as increasing fruit and vegetable intake, may be more effective than targeting certain nutrients such as mineral intake.

\section{Blood Pressure and Fruits and Vegetables Intake}

According to experimental studies, the broad spectrum of phytochemicals within fruits and vegetables appear to elicit a blood pressure lowering effect in both hypertensive and normotensive individuals (Lampe, 1999). As previously noted, the understanding of the specific effects of phytochemicals is complicated by their sheer numbers, synergistic effects, and difficulty in separating their effects from other substances in the whole foods. However, overwhelming evidence supports the protective health effects of phytochemicals found in fruits and vegetables (e.g., antioxidant capability, ability to reduce blood pressure, immune support, anti-carcinogenic properties, anti-inflammatory, anti-viral, antibacterial effects, and ability to reduce serum lipids) (Schreiner \& Huyskens-Keil, 2006).

In a prospective study examining the diets of over 41,000 women in the US, high intake of fruits and vegetables was found to be inversely correlated with systolic and diastolic blood pressure (Ascherio, Hennekens, Willett, Sacks, \& Rosner, 1996). Furthermore, this study found increased dietary fiber intake to have an inverse correlation with blood pressure, another health benefit of high fruit and vegetable consumption.

In a randomized clinical trial involving 495 subjects with systolic blood pressure of less than $160 \mathrm{mmHg}$ and diastolic blood pressure between $80-95 \mathrm{mmHg}$, it was demonstrated that a diet rich in fruits and vegetables and low-fat dairy and foods low in saturated fat was effective in reducing blood pressure for both hypertensive and 
normotensive subjects. For hypertensive subjects, blood pressure was reduced by 5.5 $\mathrm{mmHg}$ (systolic) and $3 \mathrm{mmHg}$ (diastolic), and for normotensive subjects by $3.5 \mathrm{mmHg}$ (systolic) and $2.1 \mathrm{mmHg}$ (diastolic) (Appel et al., 1997).

The blood pressure modulating effects of fruits and vegetables are not limited to whole foods alone. A randomized controlled trial utilizing commercial vegetable juice as a practical method to increase vegetable intake demonstrated that 1-2 cups of Campbell's V8 juice given to subjects daily for 12 weeks effectively reduced both systolic and diastolic blood pressure in subjects who were prehypertensive at the beginning of the study (Shenoy et al., 2010). Thus, in contrast to studies showing the blood pressure reducing effects of dietary fiber, this study showed that vegetable juice, mostly devoid of fiber, can also confer beneficial effects.

Numerous studies have also examined the effects of vegetarian diets on blood pressure. Consistent with other research, the diets of the vegetarians were richer in fruit and vegetables and they also exhibited reduced blood pressure in comparison to omnivorous subjects (Steffen et al., 2005). Furthermore, such studies have demonstrated an increase in blood pressure when red meat was introduced into vegetarian diets, thus further supporting the protective role of increased consumption of fruits and vegetables (Sacks et al., 2001). In the Coronary Artery Risk Development Study, an investigation of the 15-year incidence of elevated blood pressure in relation to plant food, dairy products and red meat, data indicated an inverse dose-response relationship with elevated blood pressure and consumption of plant foods (Steffen et al., 2005). 
...we further adjusted for selected nutrients and physiologic factors known to be associated with blood pressure in model 3. Simultaneous adjustment for these potential factors in the causal pathway attenuated the relation of EBP (elevated blood pressure) with plant food intake, thereby fully suggesting that plant food intake influenced blood pressure by its action on these nutrient and physiologic factors" (Steffen et al., 2005, p. 1173).

Since the advent of the DASH diet, there are a significant number of experimental trials investigating the effects of such eating habits. In a 3-arm randomized controlled trial of 459 subjects with blood pressure ranging from normal to hypertensive, the DASH combination diet—which is rich in fruits and vegetables and low in saturated fats and cholesterol—had a significant impact on reducing both systolic and diastolic blood pressure in all subjects, but especially African Americans and hypertensive subjects (Svetkey et al., 1999). However, the dietary modification also exhibited lowering effects on normotensive patients. Svetkey et al. (1999), noted:

Despite the greater effect in hypertensive persons, the DASH combination diet was also effective in those with high-normal blood pressure, suggesting a role for this dietary intervention in primary prevention both in clinical populations and in the general population. Individuals with high-normal blood pressure are at increased risk of blood pressure-related morbidity and mortality even though they do not have clinical hypertension" ( $p$. 292).

Another clinical trial of 412 participants randomized subjects into 2 groups consuming either a 'typical US diet' high in sodium or the DASH diet with different levels of sodium intake. After the 30-day trial, subjects eating the DASH diet exhibited lower blood pressure at all sodium intake levels in comparison to the control diet, and the participants eating the DASH diet with the lowest sodium level exhibited an average reduction in systolic blood pressure of $7.1 \mathrm{mmHg}$ for normotensive subjects and $11.5 \mathrm{mmHg}$ for hypertensive subjects 
(Sacks et al., 2001). The results of this study are significant on multiple levels.

First, it demonstrates the efficacy of the DASH diet on varying intakes of sodium, supporting its utilization and applicability in populations. Second, this study supports the blood pressure lowering effects of limiting and reducing dietary sodium. Finally, this study demonstrated that the effect of combining the DASH diet with reductions in sodium intake was more effective than either method alone, and that for hypertensive subjects 'the effects were equal to or greater than those of single-drug therapy' (Sacks et al., 2001).

Extensive experimental and prospective research supports the inclusion and increased consumption of fruits and vegetables for their health benefits, especially in lowering blood pressure and reducing mortality and morbidities associated with cardiovascular conditions. Despite medical advances permitting the use of pharmacological treatments in the control of suboptimal blood pressure, simple dietary changes and other lifestyle habit modifications are an effective alternative that not only confer benefits in reduction of risks for other chronic conditions, but reduce or negate the need for costly therapies that may have negative side effects. Craddick et al. (2003) states:

The DASH dietary pattern has proven to have beneficial effects. It may be easier than many other dietary patterns to follow because it is a wholistic diet and focuses on major food groups (fruits and vegetables, low-fat dairy, whole grains, legumes and nuts) rather than individual nutrients. It does not require purchase of unusual foods or advanced cooking knowledge or training. In addition, study participants have found it satisfying and generally acceptable" (p. 490). 
The alarming prevalence of elevated blood pressure and the resulting health dangers demonstrate a critical need for implementing practical methods to help prevent and control this debilitating condition. Efforts such as the DASH diet and suggestions for increasing fruit and vegetable consumption in US Dietary Recommendations and inclusion in public health guidelines such as the Healthy People campaign are but just a beginning in the broad efforts necessary to reach populations affected by this threat. In both developed and undeveloped nations, the current food environment has become replete with products devoid of true nutritional value, including energy dense, low fiber foods that have been heavily processed and drained of vital vitamins and minerals necessary for optimal health.

\section{Health-Related Quality of Life}

Health-Related Quality of Life (HRQOL) is a multidimensional view of assessing the health of an individual. In many ways still a fledging perspective on wellness, HRQOL has steadily been gaining ground in the last decade as a complementary method of evaluating health in individuals. In 1991 the World Health Organization began a program called WHOQOL in which a general guideline was developed to assess health beyond the basic construct of being free from disease. Currently the Constitution of the WHO defines health as "A state of complete physical, mental, and social well-being not merely the absence of disease..." (WHOQOL)

It follows that the measurement of health and the effects of health care must include not only an indication of changes in the frequency and severity of diseases but also an estimation of well-being and this can be assessed by measuring the improvement in the quality of life related to health care (WHOQOL). 
With medical and technological advances offering better cures and extension of life, measuring health in relation to presence of disease has become an antiquated method of gauging human health ("Health-Related Quality of Life"). Although still quantitative in nature, measures of HRQOL offer a more qualitative perspective of human health and tells a more comprehensive story about the wellness of a person. Another advantage of HRQOL assessment is that it is self-assessed; assessments that are administered by others may provide only an outsider's perspective on an individual's health. The Centers for Disease Control and Prevention states:

HRQOL questions about perceived physical and mental health and function have become an important component of health surveillance and are generally considered valid indicators of service needs and intervention outcomes. Self-assessed health status also proved to be more powerful predictor of mortality and morbidity than many objective measures of health. HRQOL measures make it possible to demonstrate scientifically the impact of health on quality of life, going well beyond the old paradigm that was limited to what can be seen under a microscope ("Health-Related Quality of Life").

One of the many benefits of HRQOL measures is that they recognize nuanced differences in heath that may otherwise be overlooked. Two patients with the same condition may have drastically different experiences with their illness, thus demonstrating that the traditional method of measuring health may be inadequate for assessing an individual's true experience with their ailment. The experience of health symptoms can have varying and drastic impact on the perception of wellness of an individual. 


\section{Methods}

\section{Overview}

This randomized controlled trial was conducted by recruiting a convenience sample that was randomly assigned to two separate groups: 1) a control group and 2) an intervention group, which underwent 4 consecutive weeks of nutritional therapy (i.e., daily ingestion of a Green Smoothie). Measures of blood pressure and self-assessed health quality were used to evaluate the effects of the nutritional therapy intervention by comparing data from observations before and after the 4-week trial. Each subject randomized to the Green Smoothie treatment group followed identical consumption schedules and made identical recipes.

\section{Subjects}

The study sample was recruited from the Portland, OR metropolitan area by means of social media such as Facebook and Craigslist, postings at public bulletin boards, coffee shops, universities and colleges, hospitals, grocery stores, alternative healthcare offices, and by word-of-mouth. Inclusion criteria included being over the age of 18 yrs., having the physical and financial capability to purchase ingredients and make the Green Smoothies on a daily basis, being in good health (assessed by the Santa Ana Health Questionnaire), and being available for the dates of data collection. Exclusion criteria included being pregnant, breastfeeding, or having intention to become pregnant during the course of the study; having allergies to spinach, apple, banana or chard; having symptoms related to low blood pressure or currently taking medication to control high 
blood pressure; and intention to make changes in diet or exercise habits throughout the course of the study. No gender or ethnicity restrictions were implemented.

All participants were required to complete the Santa Ana Health questionnaire that was then reviewed by a Emergency Medical Technician (EMT) to ensure each participant was in good health and eligible to participate in the study. Subjects ranged in age from 23-53 years and were predominantly White (86\% White; $14 \%$ Other). Of the 40 subjects who initially agreed to participate in the study, only 34 subjects were actually enrolled at time of the first data collection session due to scheduling conflicts. Of the 34 subjects, 5 dropped out during the course of the study (4 from the treatment group and 1 from the control group). One participant dropped out from the treatment group because of physical discomfort resulting from ingesting the smoothies, while three others dropped out because of inability to consistently follow the smoothie consumption protocol. The drop-out from the control group was unavailable for the final data collection session.

\section{Procedures}

Once the initial participant pool of 40 was recruited, subjects were randomized to either the control or treatment (i.e., Green Smoothie) group. The randomization procedure was performed by blindly drawing names printed on slips of paper. All participants were then notified of their group assignment and provided with scheduling options for initial data collection. Scheduling conflicts caused some subjects to drop out, resulting in unequal sample sizes.

Subjects randomized to the Green Smoothie group were further contacted to schedule a 'Green Smoothie Demo' session in which they were given a packet of 
information and a demonstration of the step-by-step procedures for making a Green Smoothie. The packet included the consumption schedule and daily recipes, which were identical for all participants in the treatment group. The packet also contained written directions for making smoothies, tips and suggestions for increasing palatability (e.g., cutting the greens very finely, adding ice, not diluting too much), as well as maps and schedules for local farmers' markets).

Participants in the treatment group were also given a website address for an internet blog created specifically for the purpose of this study. The blog had a question/answer forum containing smoothie and health-related questions, information about potential side-effects of smoothie consumption, articles about the benefits of Green Smoothies, and a general community area in which participants could make comments about their experiences and/or post photos of their smoothies. The purpose of this blog was to provide a sense of support and community for the treatment group participants while undertaking this nutritional intervention.

Furthermore, participants in the treatment group received weekly emails from the principal investigator offering encouragement and support throughout the duration of the study. This treatment protocol required nothing more than the consumption of 1 quart of Green Smoothie each day for 4 consecutive weeks, and participants were encouraged not to change anything else about their daily habits or diet.

After the initial data collection session, participants in the control group were instructed by the principal investigator to maintain their current diets and to look for emails that would provide information about scheduling the final data collection session. 
Data collection sessions took place from July 2, 2012 to August 24, 2012 in the College of Urban and Public Affairs Student Lounge at Portland State University on the 2nd floor of the Urban Center Building. Prior to the initial session, all participants were provided with the Santa Ana Health Survey and the Nutritional Assessment Questionnaire (NAQ) and were asked to complete the paperwork prior to the meeting. Copies were available at the session for those unable to complete them before the session. Upon arrival, all participants were given a blank note card and asked to anonymously provide a 5-digit number that would operate as their identification number for the study. Participants wrote their full name on one side of the card and their identification number on the other side. Each note card was placed in a manila folder by the participant. At a later time, an objective third party created an Excel spreadsheet connecting the identification numbers with names of participants. For the duration of the study, the principal investigator accessed data only by identification number. All participants were given two copies of the informed consent form, and one copy was signed and returned to the principal investigator and the other was retained by the subject. After completing all paperwork, participants were given a blank data sheet and waited to meet with the Emergency Medical Technician (EMT), who measured resting heart rate and resting blood pressure. Measurements were made in duplicate, and the arithmetic average was calculated and recorded for use in data analysis. After meeting with the EMT, all subjects met with a licensed personal trainer who made anthropometric measurements, including bodyweight, waist circumference, hip circumference, and skinfold measurements. All measurements were performed in duplicate, with the exception of skinfold measurements, which were made in triplicate; the arithmetic averages were 
calculated and recorded for use in statistical analysis. Participant height, age, gender and ethnicity were all self-reported. After measurements were made and recorded, subjects returned their data sheets to the principal investigator.

The final data collection session at the conclusion of the study followed a protocol identical with the initial data collection session (with the exception of the informed consent and identification note cards). Data collection sessions varied in duration, depending on the number of participants in attendance. However, total time spent by each subject at the data collection session was no longer than one hour for each session and was much shorter if paperwork had been previously completed. Subject schedules were such that not all data collection sessions could be conducted on the same day. Initial data collection sessions were conducted on 3 different days within a 7-day period; final data collection sessions required 4 different days within a 12-day period.

\section{Materials/Equipment}

Blood pressure measurements were made in duplicate using a sphygmomanometer (Mabis Healthcare, Inc. model \#09-149-011) and a standard stethoscope (Sprague). Resting heart rate was measured in duplicate using the stopwatch feature on an Apple iPhone 4S. Heart rate was measured for 30 seconds, and then multiplied by 2 to obtain beats per minute. Body weight was measured in duplicate to the nearest 0.10 lb. with a 7407 Stainless Steel Electronic Lithium Model Scale (Taylor). Circumference measurements were made in duplicate to the nearest 0.25 in. with a reinforced fiberglass flexible measuring tape (Dean). Triplicate skinfold measurements were made to the nearest $0.5 \mathrm{~mm}$ with Lange skinfold calipers (Beta Technology, Santa 
Cruz, CA). The 3-site skinfold protocol for women used the tricep, suprailium and thigh (Jackson, Pollock, \& Ward, 1980). The 3-site skinfold protocol for men used the chest, abdomen, and thigh (Jackson \& Pollock, 1978).

Other materials included the Nutritional Assessment Questionnaires, the Santa Ana Health Survey, data sheets, and a 3-panel folding privacy screen for subject privacy during skinfold measurement.

\section{Data Analysis}

During data collection sessions, subjects promptly placed the completed NAQs and data sheets into manila folders (one for each type of document) without review by the principal investigator so that anonymity was maintained. The principal investigator reviewed documents only after all documents (identified only by number) were placed in the folders. NAQs were scored with a pen and calculator, and each score was recalculated several times for accuracy. Once all NAQs were scored by the principal investigator, forms were rescored by an objective third party to verify the accuracy of scores. Excel spreadsheets were used to enter all subject data, and were organized by anthropometric data, physiological data, and NAQ scores for each group. After both data collection session were completed and data were recorded, "difference scores" (posttest pretest) were calculated for each variable.

Data were analyzed for the 29 subjects who completed the 4-week study using the software SAS version 9.2 (SAS Institute Inc.). An independent t-test was used to test for differences between the control group and treatment group at baseline. A dependent ttest was used to test for differences over time for both the control and treatment groups. 
The uncorrected $p$-value for statistical significance was originally set at $p \leq 0.05$; the Bonferroni-corrected per-comparison $\mathrm{p}$-level approximated $\mathrm{p}=0.002$. 


\section{Results}

A total of 40 volunteers were enrolled and randomized to either the treatment or control group at the beginning of the study. Twenty subjects were assigned to the control group and twenty to the treatment group. Of the initial 40 subjects, 6 dropped out before the initial data collection (schedule conflict or failure to respond to scheduled data collection session). Of the remaining 34 participants, only 29 completed the study. Four of the subjects who did not complete the study ( 3 male, 1 female) were from the treatment group; one subject (male) was from the control group. One participant withdrew due to physical discomfort resulting from ingestion of the Green Smoothies, and the three others withdrew from the treatment group because of inconsistent compliance with the Green Smoothie ingestion protocol. The subject from the control group withdrew because of a schedule conflict with the final data collection session. All data collected from eventual dropouts were excluded from analysis. Data from the 29 subjects completing the study included 13 in the control group (6 male; 7 female) and 16 in the treatment group ( 6 male; 10 female), ranging in age from 23 to 53 years.

Table 1 contains descriptive data for all participants at baseline. No statistically significant between-group differences were noted at baseline for any of the variables. 
Table 1. Subject Characteristics at Baseline

\begin{tabular}{|c|c|c|c|c|}
\hline Variable & Control & Treatment & t-value & p-value \\
\hline $\mathrm{n}$ & 13 & 16 & & \\
\hline Age (yr) & $33.7(8.9)$ & $31.6(6.3)$ & 0.73 & 0.47 \\
\hline Weight (lbs.) & $156.4(31.6)$ & $161.0(24.5)$ & -0.45 & 0.66 \\
\hline $\begin{array}{l}\text { Waist } \\
\text { Circumference } \\
\text { (in.) }\end{array}$ & $32.3(4.05)$ & $31.2(2.94)$ & 0.79 & 0.43 \\
\hline $\begin{array}{l}\text { Hip } \\
\text { Circumference } \\
\text { (in.) }\end{array}$ & $38.8(2.81)$ & $39.7(3.30)$ & -0.72 & 0.48 \\
\hline $\begin{array}{l}\text { Waist to Hip } \\
\text { Ratio }\end{array}$ & $0.83(0.06)$ & $0.80(0.08)$ & 1.14 & 0.27 \\
\hline Body Fat (\%) & $21.2(8.5)$ & $22.9(9.7)$ & -0.5 & 0.62 \\
\hline $\begin{array}{l}\text { Systolic BP } \\
(\mathrm{mmHg})\end{array}$ & $111.9(12.6)$ & $111.0(7.3)$ & 0.24 & 0.81 \\
\hline $\begin{array}{l}\text { Diastolic BP } \\
(\mathrm{mmHg})\end{array}$ & $73.69(8.3)$ & $72.9(7.8)$ & 0.25 & 0.80 \\
\hline $\begin{array}{l}\text { Resting Heart } \\
\text { Rate (bpm) }\end{array}$ & $65(5.6)$ & $67(7.2)$ & -0.85 & 0.41 \\
\hline $\begin{array}{l}\text { NAQ: Diet (0- } \\
63)\end{array}$ & $24.4(5.0)$ & $23.7(4.8)$ & 0.38 & 0.70 \\
\hline $\begin{array}{l}\text { NAQ: Lifestyle } \\
(0-12)\end{array}$ & $3.5(1.4)$ & $4.5(1.8)$ & -1.56 & 0.13 \\
\hline $\begin{array}{l}\text { NAQ 1: Upper } \\
\text { GI } \\
(0-57)\end{array}$ & $8.0(4.7)$ & $8.3(5.0)$ & -0.17 & 0.87 \\
\hline $\begin{array}{l}\text { NAQ 2: } \\
\text { Liver/Gallbladder } \\
(0-84)\end{array}$ & $11.1(8.2)$ & $8.5(6.1)$ & 0.97 & 0.34 \\
\hline
\end{tabular}




\begin{tabular}{|c|c|c|c|c|}
\hline $\begin{array}{l}\text { NAQ 3: Small } \\
\text { Intestine } \\
(0-51)\end{array}$ & $9.5(6.5)$ & $9.4(7.4)$ & 0.04 & 0.97 \\
\hline $\begin{array}{l}\text { NAQ 4: Large } \\
\text { Intestine } \\
(0-60)\end{array}$ & $6.6(6.9)$ & $5.5(6.0)$ & 0.47 & 0.645 \\
\hline $\begin{array}{l}\text { NAQ 5: Minerals } \\
(0-87)\end{array}$ & $6.7(5.2)$ & $6.8(6.3)$ & -0.05 & 0.96 \\
\hline $\begin{array}{l}\text { NAQ 6: Fatty } \\
\text { Acids } \\
(0-24)\end{array}$ & $4.2(2.4)$ & $4.0(2.4)$ & 0.17 & 0.86 \\
\hline $\begin{array}{l}\text { NAQ 7: } \\
\text { Sugar Handling } \\
(0-42)\end{array}$ & $11.9(5.3)$ & $10.3(7.0)$ & 0.69 & 0.50 \\
\hline $\begin{array}{l}\text { NAQ 8: Vitamin } \\
(0-84)\end{array}$ & $10.2(6.9)$ & $10.3(9.4)$ & -0.03 & 0.98 \\
\hline $\begin{array}{l}\text { NAQ 9: Adrenal } \\
(0-78)\end{array}$ & $14.3(9.4)$ & $12.9(10.9)$ & 0.37 & 0.71 \\
\hline $\begin{array}{l}\text { NAQ 10: } \\
\text { Pituitary } \\
(0-39)\end{array}$ & $4.0(2.5)$ & $3.1(2.7)$ & 0.95 & 0.35 \\
\hline $\begin{array}{l}\text { NAQ 11: } \\
\text { Thyroid } \\
(0-48)\end{array}$ & $4.2(4.0)$ & $4.7(4.5)$ & -0.29 & 0.78 \\
\hline $\begin{array}{l}\text { NAQ 12: Men } \\
(0-27)\end{array}$ & $1.5(1.4)$ & $0.68(1.0)$ & 1.19 & 0.26 \\
\hline $\begin{array}{l}\text { NAQ 13: Women } \\
(0-60)\end{array}$ & $13.6(5.3)$ & $7.9(4.5)$ & 2.37 & 0.03 \\
\hline $\begin{array}{l}\text { NAQ 14: Cardio } \\
(0-30)\end{array}$ & $1.31(1.25)$ & $2.13(2.1)$ & -1.25 & 0.22 \\
\hline
\end{tabular}




\begin{tabular}{|l|l|l|l|l|}
\hline $\begin{array}{l}\text { NAQ 15: Kidney } \\
(0-15)\end{array}$ & $1.54(1.61)$ & $1.44(1.71)$ & 0.16 & 0.87 \\
\hline $\begin{array}{l}\text { NAQ 16: } \\
\text { Immune } \\
0-30)\end{array}$ & $2.85(3.48)$ & $2.94(2.62)$ & -0.08 & 0.94 \\
\hline
\end{tabular}

Data are mean (std. dev.)

It was hypothesized that the 4-week nutritional intervention in this study would reduce resting blood pressure and NAQ scores. Additional data (i.e., resting heart rate, waist and hip circumference, skinfold thickness) were collected to further explore the potential effects of daily Green Smoothie consumption. Table 2 shows the mean changes in each variable for each group separately. Also included are the p-values for each statistical test.

Table 2. Mean Changes in Study Variables in Control and Treatment Groups

\begin{tabular}{|l|l|l|l|}
\hline Variable & & Control & Treatment \\
\hline Bodyweight (lbs.) & Mean & -0.40 & -0.18 \\
\hline & p-value & 0.62 & 0.82 \\
\hline $\begin{array}{l}\text { Waist Circumference } \\
\text { (in.) }\end{array}$ & Mean & -0.37 & -0.49 \\
\hline & p-value & 0.06 & 0.03 \\
\hline Waist-to-Hip Ratio & Mean & -0.01 & -0.02 \\
\hline & p-value & 0.16 & 0.05 \\
\hline Body Fat (\%) & Mean & -1.23 & -0.63 \\
\hline & p-value & 0.06 & 0.30 \\
\hline Systolic BP (mmHg) & Mean & 2.54 & 1.41 \\
\hline & p-value & 0.34 & 0.57 \\
\hline Diastolic BP (mmHg) & Mean & 0.69 & -1.44 \\
\hline
\end{tabular}




\begin{tabular}{|c|c|c|c|}
\hline & p-value & 0.76 & 0.41 \\
\hline \multirow[t]{2}{*}{ Heart Rate (bpm) } & Mean & 4.23 & 3.06 \\
\hline & $p$-value & 0.22 & 0.29 \\
\hline \multirow[t]{2}{*}{ Diet } & Mean & $\begin{array}{l}-1.62 \\
\end{array}$ & -2.31 \\
\hline & p-value & 0.26 & 0.04 \\
\hline \multirow[t]{2}{*}{ Lifestyle } & Mean & -0.38 & -0.69 \\
\hline & p-value & 0.51 & 0.13 \\
\hline \multirow[t]{2}{*}{ 1: Upper GI } & Mean & 1.54 & -0.50 \\
\hline & p-value & 0.17 & 0.73 \\
\hline \multirow[t]{2}{*}{ 2: Liver/Gall } & Mean & \begin{tabular}{|c|}
-1.31 \\
\end{tabular} & $\begin{array}{l}-1.00 \\
\end{array}$ \\
\hline & p-value & 0.51 & 0.48 \\
\hline \multirow[t]{2}{*}{ 3: Sm. Intestine } & Mean & -0.69 & -1.88 \\
\hline & p-value & 0.57 & 0.04 \\
\hline \multirow[t]{2}{*}{ 4: Lg. Intestine } & Mean & -1.62 & -2.63 \\
\hline & p-value & 0.19 & 0.05 \\
\hline \multirow[t]{2}{*}{ 5: Minerals } & Mean & -1.00 & $\begin{array}{l}-1.38 \\
\end{array}$ \\
\hline & p-value & 0.41 & 0.04 \\
\hline \multirow[t]{2}{*}{ 6: Fatty Acids } & Mean & 0.77 & -0.38 \\
\hline & p-value & 0.39 & 0.54 \\
\hline \multirow[t]{2}{*}{ 7: Sugar } & Mean & -1.08 & 0.31 \\
\hline & p-value & 0.30 & 0.76 \\
\hline \multirow[t]{2}{*}{ 8: Vitamin need } & Mean & -0.08 & -2.75 \\
\hline & p-value & 0.97 & 0.26 \\
\hline \multirow[t]{2}{*}{ 9: Adrenal } & Mean & -0.54 & -0.20 \\
\hline & p-value & 0.80 & 0.26 \\
\hline \multirow[t]{2}{*}{ 10: Pituitary } & Mean & -1.31 & -0.81 \\
\hline & p-value & 0.20 & 0.31 \\
\hline 11: Thyroid & Mean & -0.23 & -0.19 \\
\hline
\end{tabular}




\begin{tabular}{|l|l|l|l|}
\hline & p-value & 0.83 & 0.88 \\
\hline 12: Men & Mean & 1.00 & -0.17 \\
\hline & p-value & 0.39 & 0.36 \\
\hline $13:$ Women & Mean & -3.86 & 1.00 \\
\hline & p-value & 0.06 & 0.46 \\
\hline 14: Cardio & Mean & 0.08 & -0.19 \\
\hline & p-value & 0.81 & 0.78 \\
\hline $15:$ Kidney & Mean & 0.31 & -0.50 \\
\hline & p-value & 0.39 & 0.25 \\
\hline $16:$ Immune & Mean & 0.00 & 0.19 \\
\hline & p-value & 1.00 & 0.81 \\
\hline
\end{tabular}

Figures 1-6 illustrate between-group comparisons of pretest-posttest difference scores for selected variables (i.e., waist circumference, waist-to-hip ratio, and NAQ diet, small intestine, large intestine, and mineral needs subsections). Comparisons for all other subsections of the NAQ and other physiologic measures can be found in Appendix H.

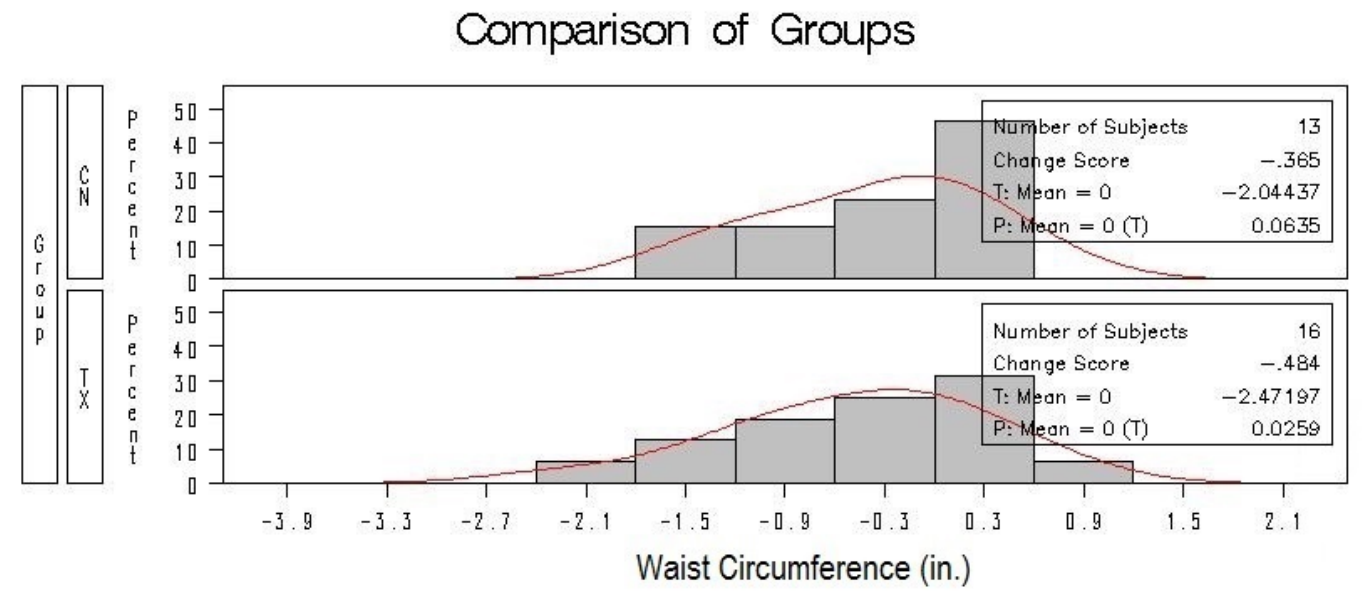

Figure 1. Comparison of Control and Treatment Group Waist Circumference Pre-Post Difference Scores 


\section{Comparison of Groups}

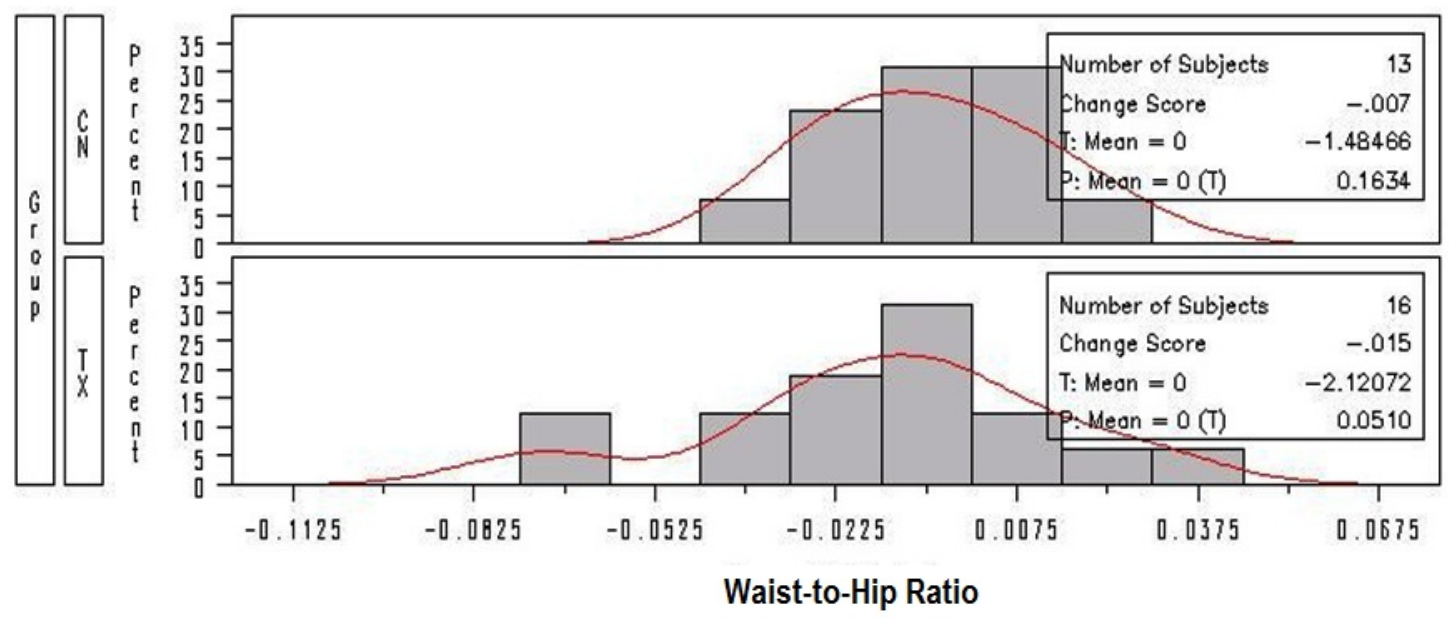

Figure 2. Comparison of Control and Treatment Group Waist-to-Hip Ratio Pre-Post Difference Scores

Comparison of Groups

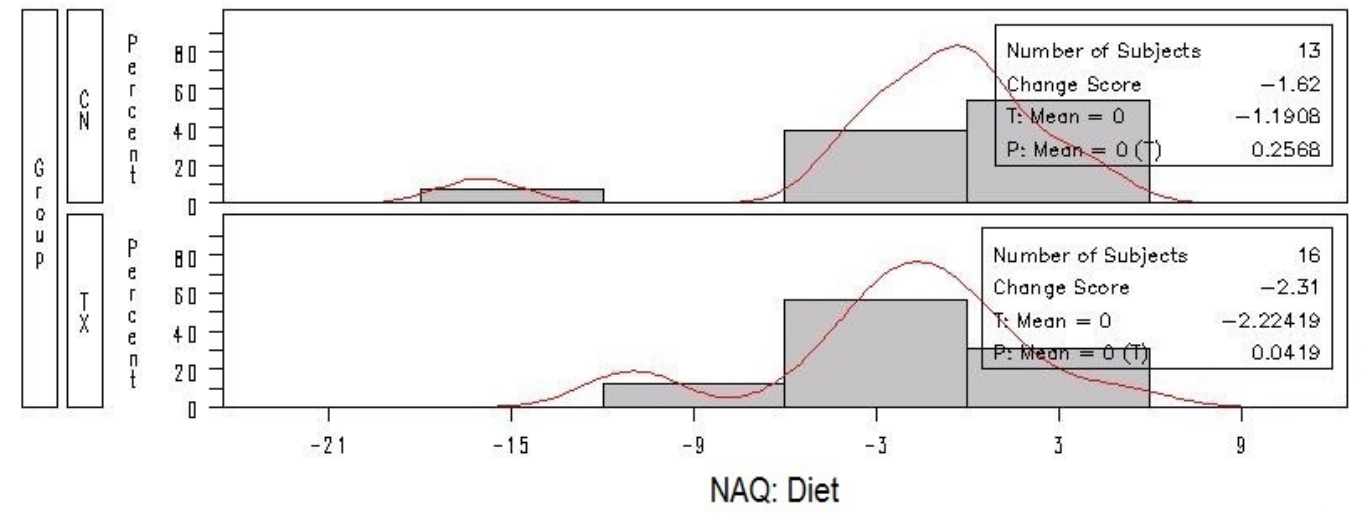

Figure 3. Comparison of Control and Treatment Group NAQ Diet Subsection Pre-Post Difference Scores 


\section{Comparison of Groups}
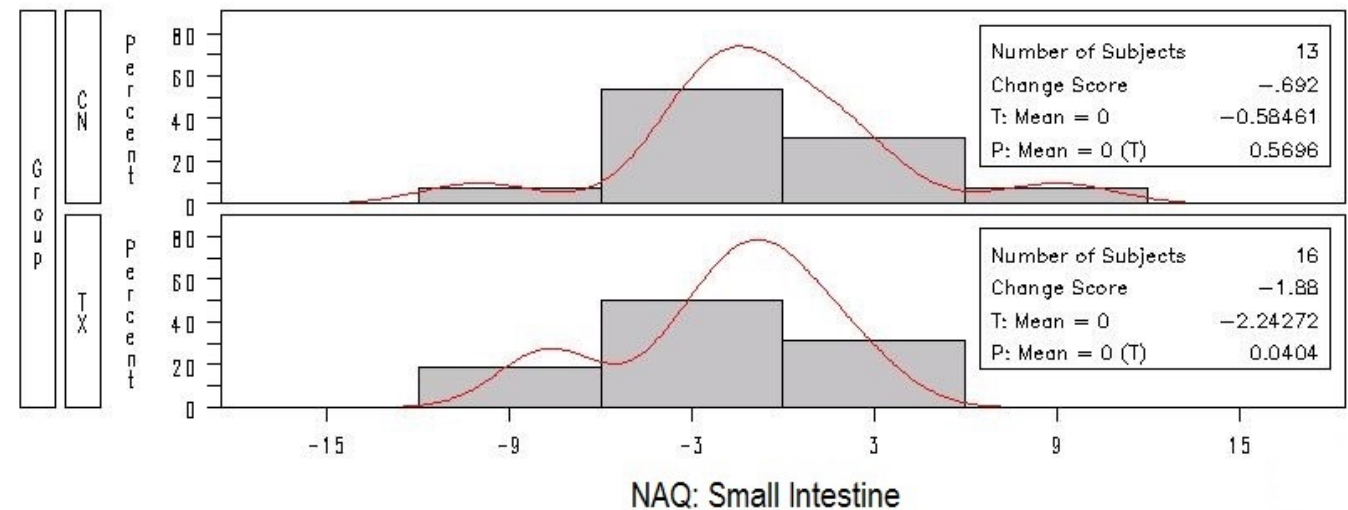

Figure 4. Comparison of Control and Treatment Group NAQ Small Intestine Subsection Pre-Post Difference Scores

Comparison of Groups
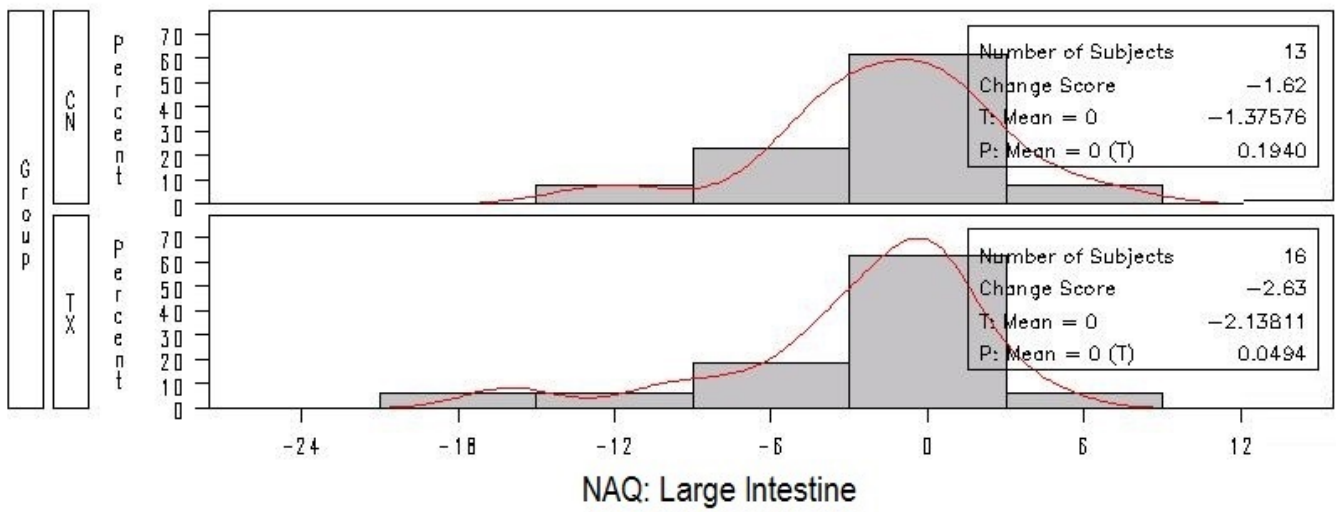

Figure 5. Comparison of Control and Treatment Group NAQ Large Intestine Subsection Pre-Post Difference Scores. 
Comparison of Groups

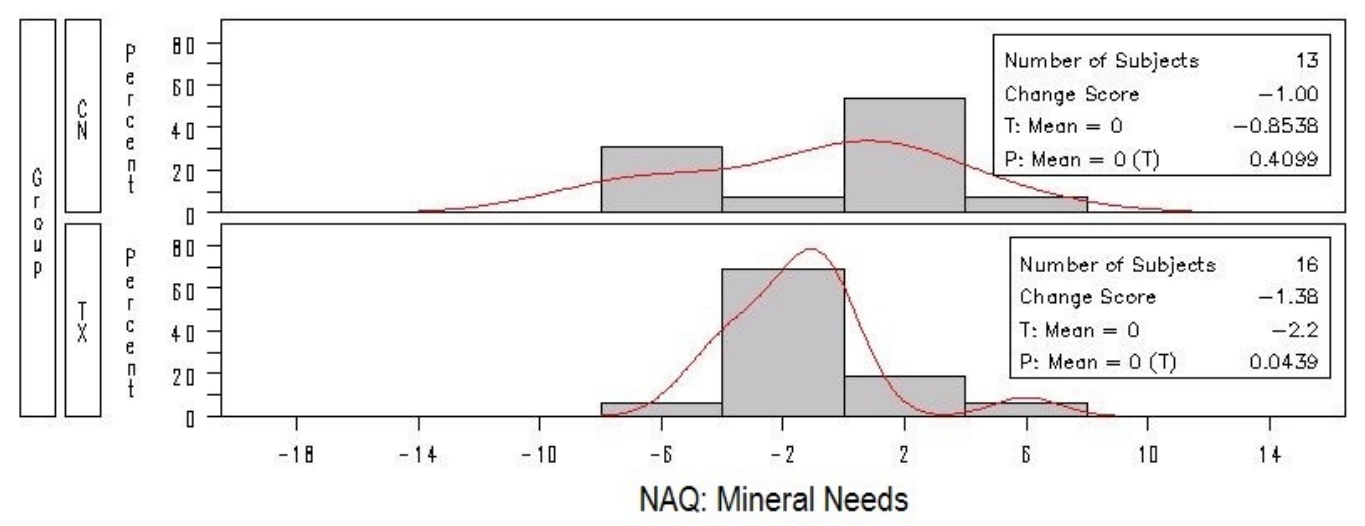

Figure 6. Comparison of Control and Treatment Group NAQ Mineral Needs Subsection Pre-Post Difference Scores 


\section{Discussion}

The aim of this nutritional therapy study was to investigate the effects of a fourweek nutrition intervention on several physiologic/anthropometric measures and healthrelated quality of life. Although statistically significant changes were not observed, the results from this study provide preliminary data to suggest a beneficial effect of Green Smoothie consumption on 1) waist circumference and waist-to-hip ratio, and 2) healthrelated quality of life as measured by the diet, small intestine, large intestine and mineral needs subsections of the NAQ.

Results of this study indicate that pretest-posttest differences did not reach statistical significance in the nutrition intervention group for blood pressure or healthrelated quality of life. However, waist circumference, waist-to-hip ratio, and several NAQ subsections (i.e., diet, small intestine, large intestine, and mineral needs) showed potentially meaningful reductions in the treatment group. It is important to note that the lack of statistical significance should be interpreted in lieu of the multiple hypothesis tests, which were conducted with Bonferroni-corrected critical p-values. Given the exploratory nature of this research, however, it was felt that attention should be given to several variables that provided preliminary evidence to support daily Green Smoothie consumption.

\section{Blood Pressure}

The mean changes in both systolic and diastolic blood pressure for each group was examined in this study because previous research has shown that increased 
consumption of fresh fruits and vegetables can reduce both systolic and diastolic blood pressure (Craddick et al., 2003; Lampe, 1999; Sacks et al., 2001; Steffen et al., 2005).

All subjects at baseline were considered to be normotensive and no statistically significant changes were seen in either group after the 4-week study. Despite extensive research demonstrating that fruit and vegetable consumption can provide beneficial effects on diastolic and systolic blood pressure - even in normotensive individuals - such an effect was not seen in this study. The lack of a statistically significant change may have been due to a number of factors, including non-compliance or inconsistent compliance with the smoothie consumption protocol, insufficient treatment duration, or non-compliance with restrictions on exercise and caffeine intake 2 hours before measurements. Insufficient power (i.e., sample size) is also a possibility.

Blood pressure was included as a dependent measure because it is a simple method for evaluating the physiological health of the cardiovascular system. Blood pressure measurement provides insight into the health of the heart, blood vessels and resistance to blood flow that may indicate possible health problems requiring treatment (Perloff et al., 1993). Resting blood pressure outside the range of expected 'normal' for an individual greatly increases the risk of developing cardiovascular disease, the most common cause of death in developed countries. Thus, the treatment and prevention of high blood pressure is a simple and critical approach for the protection of health and well-being of the population (Anderson \& Horvath, 2004; Appel, 1999; Strandberg et al., 2001). 


\section{Waist Circumference and Waist-to-Hip Ratio}

Research indicates that waist circumference and the waist-to-hip ratio are considered to be accurate and informative indicators of cardiovascular disease risk (Dobbelsteyn, Joffres, MacLean, Flowerdew \& The Canadian Heart Health Surveys

Research Group, 2001; Elsayed et al., 2008). According to the Olivetti Heart study (Siani et al., 2002), waist circumference is highly positively correlated with increased blood pressure, indicating a major role of central adiposity in the expression of conditions such as the metabolic syndrome (hypertension, dyslipidemia, central obesity, and insulin resistance). Sung and Ryu (2004) also concluded that waist circumference operates as an independent indicator of blood pressure in a cross-sectional survey study of over 50,000 Koreans. The body mass index (BMI) is calculated using measures of weight and height, but does not take into consideration bone or muscle mass, thus often categorizing muscular individuals as 'overweight'. Waist-to-hip ratio and waist circumference have recently become well recognized in the health field as being accurate indicators of health risk and are currently replacing measures of BMI as appropriate measures of health risk (Dobbelystn et al., 2001; Elsayed et al., 2008).

One reasonable explanation for the apparent reduction in waist circumference and waist-to-hip ratio in the treatment group is that consumption of Green Smoothies affected total dietary intake in addition to bolstering the diet with increased fruits and leafy green vegetables, increased fiber intake, and overall water intake. Not only would the fresh fruits and vegetables confer health benefits, but the increased fiber intake could result in 
an increased feeling of "fullness" or satiation in the treatment group. This may have resulted in reduced consumption of food overall in this group.

Uncontrolled factors (e.g., seasonal dietary and/or physical activity variation, attempts to lose weight, etc.) may also have influenced waist circumference and waist-tohip ratio. However, the fact that subjects were assigned randomly to groups makes this explanation unlikely.

\section{Health-Related Quality of Life}

The traditional characterization of health in the United States has often been limited to merely the absence of disease; this overlooks other variables that may contribute to health and well-being that are considered in other approaches (e.g., Eastern and holistic views of health).

...Health in the United States has traditionally been measured narrowly and from a deficit perspective, often using measures of morbidity and mortality. But health is seen by the public health community as a multidimensional construct that includes physical, mental and social domains ("Health-related quality of life").

In an effort to encompass a broader definition of health, the Nutritional

Assessment Questionnaire (NAQ) - a 322 question self-report health survey - was used to assess health-related quality-of-life (HRQOL). This was intended to allow the detection of nuanced changes in subjects' perceived symptoms of burden that otherwise may have been overlooked in more conventional assessments of health status. The NAQ and similar surveys are often used with new patients as initial medical intake forms in naturopathic medicine for the assessment of wellness. 
Whereas community-level HRQOL addresses socioeconomic burdens and satisfaction with employment and other such factors affecting mental health, individuallevel HRQOL - as used in this study - examines health symptoms of burden such as sleep quality, physical discomfort (not necessarily physical disability), energy level, intestinal function, eating habits, sleep patterns, mood, healthy habits and other various signs of wellness. Seemingly minor changes in such symptoms can significantly impact overall quality of life on a day-to-day basis (Calvert \& Freemantle, 2003). This can both negatively and positively impact an individual's overall experience of daily life, and can have long-term impacts on a person's health and vulnerability to disease. Poor sleep and unhealthful eating habits may leave the immune system vulnerable to viruses and disease, which can negatively affect the long-term health of individuals. Reduced energy levels and poor moods can have a negative impact on productivity, resulting in possible detrimental effects on employment status, sense of self, and eventual socioeconomic status. Broader considerations such as these demonstrate that viewing human health as merely an absence of disease or reduction in morbidity is reductive and neglectful of the many aspects of healthy living that greatly contribute to the well-being and health of the human population (Guyatt, Feeny, \& Patrick, 1993; Koch, 2000).

\section{Nutritional Assessment Questionnaire}

Although the use of extensive health questionnaires such as the NAQ is prevalent in naturopathic medicine and Eastern healing practices, their use is limited in Western practices. Furthermore, the few studies that have used the NAQ-or instruments similar to it-have done so with elderly or ailing patients and to date appear not to have been 
utilized in assessing symptoms of burden in healthy subjects in the published studies reviewed for this research. However, with the growing interest in and acknowledgement of the importance of health-related quality of life in assessing holistic health, utilization of surveys such as the NAQ may prove helpful in the healthcare system in gaining a deeper sense of wellness and health for patients. Such instruments provide an opportunity for the detection of nuanced changes that can have significant effects on health, but may be easily overlooked by more traditional measures of wellness (e.g., presence of morbidities and mobility issues).

\section{NAQ: Diet}

The 'Diet' subsection of the NAQ evaluates general eating and drinking habits that relate to overall health such as frequency of caffeine intake, alcohol consumption, dieting, frequency of fast food intake, and consumption of processed meats, flour and refined sugar. The slight reduction in this score exhibited by the treatment group suggests an improvement in general habits of eating and drinking following the 4 weeks of Green Smoothies.

Based on the quantitative nature of this study, one can only speculate about the reasons behind the apparent change in this score in the treatment group. First, it is possible that the treatment group had reduced frequency of undesirable dietary habits due to increased sensations of "fullness" (satiety) resulting from smoothie consumption. Each day, the treatment group was required to ingest 1 quart of the Green Smoothies, which contained additional natural fiber that may have contributed to feelings of "fullness." Another factor may have been conscious decisions made by the subjects as a 
result of their involvement in the study. It is possible that subjects in the treatment group were more likely to moderate their poor dietary choices because they wanted to use this study as an opportunity to improve their health. Finally, it is possible that an element in the smoothies (i.e., fiber, vitamins and minerals, or phytochemicals) - alone or in some combination - reduced their desire to engage in detrimental dietary habits.

\section{NAQ: Small and Large Intestine}

The 'Small Intestine' subsection of the NAQ evaluates symptoms related to digestion, such as post-meal bloating, food allergies and sensitivities, mental sluggishness, cravings and nightmares. A reduction in score for the 'Large Intestine' section demonstrates an improvement in symptoms related to bowel sensitivity and regularity, strong body odors, frequency of dark circles under the eyes, and coated tongues. The slight reduction in this score exhibited by the treatment group suggests improvements can be made in this area among those consuming smoothies.

It is widely understood that adequate fiber consumption is important for maintaining healthy digestive function (Anderson et al., 1994; Anderson et al., 2009; Theuwissen \& Mensink, 2008). It is also known that the majority of the US population is still deficient in daily dietary fiber consumption, thus leading to digestive conditions such as intestinal polyps, constipation, irritable bowel syndrome, and weight gain (Clemens et al., 2012). Thus, any effect of Green Smoothie consumption on intestinal health may be due to the increased fiber content of the beverage, which would improve digestive function and reduce the severity and frequency of the symptoms of burden associated with poor intestinal function. 


\section{NAQ: Mineral Needs}

A slight reduction in the score on the 'Mineral Needs' section of the NAQ suggests that the treatment group reduced the frequency and severity of symptoms of burden, such as hoarseness of voice, fevers, rashes, joint issues, anemia and loss of sense of smell. According to the NAQ, these symptoms are linked to inadequate intake of essential minerals.

There are 16 essential minerals the human body requires for proper function. The low intake of fruits and vegetables in the US - along with the current practice of fortifying many processed foods - provides evidence that the majority of the population obtains inadequate vitamins and minerals from the food they consume. The slight apparent reduction of symptoms of burden linked to 'Mineral Needs' found in this investigation suggests that daily Green Smoothie consumption for 4 weeks may have improved the ability to meet mineral needs and reduced the frequency and severity of many of the symptoms related to inadequate mineral intake.

To date, organizations such as the Centers for Disease Control and Prevention and the World Health Organization have utilized self-report health surveys such as the SF-36 and the shortened SF-12, the Quality of Well Being Scale, and the Sickness Impact Profile to evaluate HRQOL in individuals (Bonomi, Patrick, Bushnnell, \& Martin, 1997; "Health-related quality of life"). Although these instruments are commonly used for HRQOL assessment in the efforts of public health, they are limited by their focus on physical mobility, frequency of illness, social activity, employment and ambulation. Among these measurement tools, the Sickness Impact Profile includes sections related to 
emotional behavior, sleep and rest, alertness and eating, which are most closely related to the changes that were hypothesized to occur in the current study. However, due to the short duration of this study and the relatively small changes that were expected to result from daily Green Smoothie consumption, these more commonly used instruments were deemed to be inadequate. The NAQ is less commonly used and is significantly lengthier than other HRQOL questionnaires, but it has the capability of detecting minor changes in distinct body systems, including gender-specific symptoms of burden.

\section{Conclusion}

The results of this investigation are in line with previous research on the benefits of fruits and vegetables and their beneficial health effects of lowering risks for chronic conditions such as cardiovascular disease. This study provides some support for the benefits of including fruits and vegetables in the regular diet, including potentially meaningful changes in risk factors such as waist circumference and waist-to-hip ratio. It also suggests that such risk factors can be modified with only slight changes to fruit and vegetable intake in as short a duration as 4 weeks.

The improved health-related quality of life suggested by the reduced symptoms of burden in this investigation complements the purported cardiovascular disease risk reduction effects of Green Smoothie consumption. The results of this investigation suggest that the introduction of Green Smoothies into the regular diet may enhance health quality of life for healthy individuals. 
Currently the burden of chronic illness dwarfs all other causes of death globally, and as much as $30 \%$ of those chronic illnesses are reported as being preventable by diet and lifestyle changes ("Chronic Disease and Health Promotion"). The results of this study suggest that daily consumption of Green Smoothies may be a practical and effective method of increasing fruit and vegetable consumption and confer beneficial health effects and possibly reduce the risk of chronic disease. Program-based public health efforts to increase fruit and vegetable consumption such as the 'Gimme 5', 'Five a Day for Better Health' and the Healthy People campaign are targeted at school-aged children and fail to have a significant impact on the general population. These programs also fail to offer tangible strategies for increasing fruit and vegetable consumption beyond giving strong recommendations for increased consumption.

Albeit a far cry from a 'magic bullet' solution sought by many, the habitual integration of Green Smoothies in the diet may offer health effects that could greatly reduce the incidence of chronic disease worldwide. Ingestion of Green Smoothies may not only help to reduce the risk for those already suffering from chronic ailments, but can also be used as a primary preventative strategy. With escalating losses in productivity, death and disability, and debilitating costs in healthcare resulting from chronic disease, the need for practical and cost-effective methods for tackling many of these preventable conditions should be at the forefront of concern for health officials and health policy makers. 


\section{Limitations}

This study was significantly limited by lack of funding, which presumably would have increased the scope and reliability of this research project. The most difficult challenge was obtaining a sample that was sufficiently large and diverse enough to characterize the 'general population'. As with many research studies that rely on volunteers, participation is often limited to individuals with adequate time to devote to compliance with the study requirements. This potentially excludes a significant portion of the population that may otherwise benefit from the research, thereby reducing the external validity of the study. Thus, without funding and the capability to adequately compensate participants, this study was dependent on a small sample of volunteers gathered through social media, public postings and word of mouth. Participants were recruited from the Portland, Oregon metropolitan area-a geographic region considered to be characterized by high health awareness - which may have reduced the effect of the intervention (i.e., participants may already have possessed relatively healthy eating and living habits). Furthermore, the sample pool was homogenous and lacked significant diversity not only culturally, but also socioeconomically. Finally, despite initial interest in participating in the study, some potential subjects were deterred by the cost of the ingredients of the smoothies and thus could not participate.

As with any controlled intervention study that does not include strict subject supervision or control by the research team, protocol compliance can be a risk to the integrity of the study. Although subjects were advised to follow daily smoothie recipes and schedules and to 'double up' the following day if an interruption in daily 
consumption occurred, the research team had to rely on the honesty of the subjects. It is possible that adherence could have been enhanced if subjects were better compensated for their participation. Without funding, participation and completion of the study offered only a chance to win one of three prizes in a random drawing. The incentive to participate in this study may have provided only a minimal sense of obligation to strictly adhere to the study protocol.

Due to the short duration of this study, all facets must be considered, and the lack of designation of organic versus non-organic produce may have had an impact on outcomes. Once again, without funding to supply the resources for this study, participants in the Green Smoothie intervention group were free to purchase producewhether organic or not-for use in making the smoothies.

Another limitation that may have affected the reliability of results was the inability to schedule posttest measurements at the same time and day of the week for each participant. Ideally, measurement of bodyweight, blood pressure, skinfolds, circumferences, and resting heart rate should take place at the same time of day to minimize diurnal variation. However, scheduling of data collection sessions was based purely on subject availability. Furthermore, despite requests that subjects abstain from exercise and caffeine at least two hours prior to data collection sessions, it is possible that the protocol was not followed, thereby influencing some physiological measures (e.g., heart rate, blood pressure). For the final round of data collection, there were three participants in the treatment group who were unavailable for scheduled sessions earlier in the week and therefore had to wait another 6 to 12 days before posttest measurements 
could be made. As advised by the principal investigator, these individuals continued to consume Green Smoothies daily until the final data collection session. A re-analysis of the treatment group pre-post data (excluding these three individuals) showed that the suggested beneficial effect of the smoothie consumption was attenuated. This suggests that a longer duration of smoothie consumption may have resulted in statistically significant changes in the variables that only approached statistical significance in this study.

The Nutritional Assessment Questionnaire, albeit thorough and perhaps the best available method for measuring symptoms of burden - at no cost to the user-was not without limitations. The questionnaire touched only briefly on symptoms such as sleep quality, energy level, general moods, appetite, and digestive regularity; even extreme changes in some of these areas would have only a slight impact on the overall scores tabulated from the entire questionnaire. This study could have benefitted by the creation of a more specific survey that explored these symptoms more precisely. Furthermore, any self-report data comes with inherent issues of quality of self-awareness (i.e., underor over-reporting). As demonstrated in previous research involving food recall, subjects tend to underreport unhealthful eating habits and over report healthful habits (Hansson, Galanti, \& Bergstrom. (2000). Thus, it is reasonable to assume that participants may have over-reported favorable habits and under-reported unfavorable tendencies when they completed the NAQ. Data from this study indicated a marked difference in NAQ scores between men and women, with men exhibiting lower scores overall. Although it is possible that the men in this study experienced fewer symptoms of burden, it is also possible that women tend to be more "in tune" with their bodies and had a better sense of 
nuanced ailments or symptoms of burden, a finding that has been observed in other studies using self-assessed measures (Hansson et al., 2000). Thus, this potential gender effect may have affected NAQ score results.

The multiple hypothesis tests resulting from utilization of the NAQ and its many subsections likely had a negative impact on the statistical power in this study. As mentioned previously, future replications of this study should consider using a more concise instrument that would reduce the number of hypothesis tests. Such an instrument might improve the ability to detect treatment effects related to specific symptoms of burden.

\section{Directions for Future Research}

Replication of this study on a larger scale, for a longer period of time, and with a more diverse population to increase generalizability of the results should be considered. Such replication would expand the impact of this study. Slight but potentially meaningful changes were observed in the short time and small sample used for this pilot study. It is not unrealistic to suggest that significant changes might be observed in both blood pressure and NAQ scores with a larger sample or longer intervention period. This is particularly true if the volunteers used in this study were more health-conscious than the general population of the United States.

A mixed-methods approach study design should be considered. Although the randomized controlled trial method is often considered to be optimal, incorporating a qualitative approach that offers subjects the ability to share their experiences about the 
consumption of smoothies could also enhance the quality of the data. It would also be useful to create a short survey investigating detailed changes in sleep quality, energy level, general mood, digestion, etc. to further explore health symptoms most likely believed to be affected from such a dietary change.

Adequate funding for a replication of this study would permit compensation that would presumably increase compliance with the study protocol. Furthermore, supplying subjects with the ingredients necessary for making the smoothies and ensuring that all participants received the same quality of produce would reduce the variability in Green Smoothie ingredients.

Future studies would also benefit by requiring that all participants arrive at the data collection sessions and fill out the paperwork at one time, thereby allowing adequate time to both mentally and physiologically relax before their sessions for physiological and body measurements.

Judging by the process of literature review for this study, it appears that despite the numerous nutritional studies investigating the benefits of fruits and vegetables, there is a dearth of studies that look specifically at vegetables, and leafy greens in particular. Current investigations into leafy greens have demonstrated them to be highly beneficial to health offering many protective effects superior to many other fruits and vegetables, thus leaving a broad area of nutrition to be explored. As previously noted, the phytochemicals in fruits and vegetables pose difficulty in the study of their purported effects, thus often showing null effects when studied individually by extraction and isolation from the foods from which they come. It may be likely that the health benefits 
of leafy greens will also be hard to pinpoint because - similar to other plant foods - they confer synergistic effects that are difficult to isolate. 


\section{References}

Anand, P., Kunnumakara, A.B., Sundaram, C., Harikumar, K.B., Tharakan, S.T., Lai, O.S., Sung, B., \& Aggarwal, B.B. (2008). Cancer is a preventable disease that requires major lifestyle changes. Pharmaceutical Research, 25 (9), 2097- 2116.

Anderson, G., \& Horvath, J. (2004). The growing burden of chronic disease in America. Health Reports, 119, 263- 270.

Anderson, J.W., Baird, P., Davis Jr., R.H., Ferreri, S., Knudtson, M., Koraym, A., Waters, V., \& Williams, C.L. (2009). Health benefits of dietary fiber. Nutrition Reviews, 67 (4), 188-205.

Anderson, J.W., Smith, B.M., \& Gustafson, N.J. (1994). Health benefits and practical aspects of high-fiber diets. American Journal of Clinical Nutrition, 59 (suppl.), $1242 \mathrm{~S}-1247 \mathrm{~S}$.

Appel, L.J. (1999). Nonpharmacologic therapies that reduce blood pressure: A fresh perspective. Clinical Cardiology, 22 (Suppl. III), III1-III5.

Appel, L.J., Brands, M.W., Daniels, S.R., Karanja, N., Elmer, P.J., \& Sacks, F.M. (2006). Dietary approaches to prevent and treat hypertension: A scientific statement from the American Heart Association. Hypertension, 47, 296-308.

Appel, L.J., Moore, T.J., Obarzanek, E., Vollmer, W.M., Svetkey, L.P., Sacks, F.M., Bray, G.A., Cogt, T.M., Cutler, J.A., Windhauser, M.M., Lin, P., \& Karanja, N. (1997). A clinical trial of the effects of dietary patterns on blood pressure. The New England Journal of Medicine, 336 (16), 1117- 1124.

Ascherio, A., Hennekens, C., Willett, W.C., Sacks, F., \& Rosner, B. (1996). Prospective study of nutritional factors, blood pressure, and hypertension among US Women. Hypertension, 27I (5), 1065.

Bazzano, L.A., He, J., Odgen, L.G., Loria, C.M., Vupputuri, S., Myers, L., \& Whelton, P. (2002). Fruit and vegetable intake and risk of cardiovascular disease in US adults: The first National Health and Nutrition Examination Survey epidemiologic follow-up study. American Journal of Clinical Nutrition, 76, 93-99.

Blatt, A.D., Roe, L.S., \& Rolls, B. (2011). Hidden vegetables: An effective strategy to reduce energy intake and increase vegetable intake in adults. American Journal of Clinical Nutrition, 93, 756- 763.

Bonomi, A.E., Patrick, D.L., Bushnell, D.M., \& Martin, M. (1997). Validation of the United States' version of the World Health Organization Quality of Life (WHOQOL) instrument. Journal of Clinical Epidemiology, 53 (1), 1-12. 
Broadhurst, C.L. (1997). Balanced intakes of natural triglycerides for optimum nutrition: An evolutionary and phytochemical perspective. Medical Hypotheses, 49, $247-$ 261.

Broekmans, W.M.R., Klopping-Ketelaars, W.A.A., Kluft, C., Van den Berg, H., Kok, F.J., \& van Poppel, G. (2001). Fruit and vegetables and cardiovascular risk profile: A diet controlled intervention study. European Journal of Clinical Nutrition, 55, 636- 642.

Brown, M.J., Ferruzzi, M.G., Nguyen, M.L., Copper, D.A., Eldridge, A.L., Schwartz, S.J., \& White, W.S. (2004). Carotenoid bioavailability is higher from salads ingested with full-fat than with fat-reduced salad dressings as measured with electrochemical detection. American Journal of Clinical Nutrition, 80, 396-403.

Cakir, H., \& Pinar, R. (2006). Randomized controlled trial on lifestyle modification in hypertensive patients. Western Journal of Nursing Research, 28 (2), 190-209.

Calvert, M.J., \& Freemantle, N. (2003). Use of health-related quality of life in prescribing research. Part 1: Why evaluate health-related quality of life? Journal of Clinical Pharmacy and Therapeutics, 28, 513-521.

"Cardiovascular Disease: Fact Sheet” World Health Organization. n.a. Web. 18 Oct. 2012.

Casagrande, S.S., Wang, Y., Anderson, C., \& Gary, T.L. (2007). Have Americans increased their fruit and vegetable intake? Trends between 1988 and 2002. American Journal of Preventative Medicine, 32 (4), 257- 263.

"Chronic Disease and Health Promotion." Center for Disease Control and Prevention. Web. 18 Oct. 2012.

Chu, Y., Sun, J., Wu, X, \& Liu, R.H. (2002). Antioxidant and antiproliferative activities of common vegetables. Journal of Agriculture: Food Chemistry, 50, 6910- 6916.

Chun, O.K., Kim, D., Smith, N., Schroeder, D., Han, J.T., \& Lee, C.Y. (2005). Daily consumption of phenolics and total antioxidant capacity from fruit and vegetables in the American diet. Journal of the Science of Food and Agriculture, 85 (10), 1715-1724.

Clemens, R., Kranz, S., Mobley, A.R., Nicklas, T.A., Raimondi, M.P., Rodriguez, J.C., Slavin, J.L., \& Warshaw, H. (2012). Filling America's fiber intake gap: Summary of a Roundtable to Probe Realistic Solutions with a Focus on Grain-Based foods. Journal of Nutrition. Retrieved from http:// http://jn.nutrition.org/content/142/7/1390S.full.pdf + html

Cottee, P. (1999). Tackling diet-related disease by promoting fruit and vegetables. Nutrition \& Food Science, 4, 173- 177. 
Craddick, S.R., Elmer, P.J., Obarzanek, E., Vollmer, W.M., Svetkey, L.P., Swain, M.C. (2003). The DASH diet and blood pressure. Current Atherosclerosis Reports, 5, 484- 491.

Dobbelsteyn, C.J., Joffres, M.R., MacLean, D.R., Flowerdew, G., \& The Canadian Heart Health Surveys Research Group. (2001). A comparative evaluation of waist circumference, waist-to-hip ratio and body mass index as indicators of cardiovascular risk factors: The Canadian Heart Health Surveys. International Journal of Obesity, 25, 652- 661.

Dutta, D., Chaudhuri, U.R., \& Chakraborty, R. (2005). Structure, health benefits, antioxidant property and processing and storage of carotenoids. African Journal of Biotechnology, 4 (13), 1510- 1520.

Elsayed, E.F., Weiner, D.E., Salem, D., Levey, A.S., Sarnak, M.J., Tighiouart, H., \& Griffith, J. (2008). Waist-to-Hip and body mass index as risk factors for cardiovascular events in CKD. American Journal of Kidney Disease, 52 (1), 4957.

Ferdinand, K.C., Patterson, K.P., Taylor, C., Fergus, I.V., Nasser, S.A., \& Ferdinand, D.P. (2012). Community-based approaches to prevention and management of hypertension and cardiovascular disease. The Journal of Clinical Hypertension, 14 (5), 336- 343.

Ferguson, L.R. (1999). Prospects for cancer prevention. Mutation Research, 428, 329338.

Feskanich, D., Ziegler, R.G., Michaud, D.S., Giovannucci, E.L., Speizer, F.E., Willett, W.C. et al. (2000). Prospective study of fruit and vegetable consumption and risk of lung cancer among men and women. Journal of the National Cancer Institute, 92, 1812-1823.

Franke, A.A., Cooney, R.V., Henning, S.M., \& Custer, L.J. (2005). Bioavailability and antioxidant effects of orange juice components in humans. Journal of Agricultural Food Chemistry, 53 (13), 5170- 5178.

Fraser, G.E. (2009). Vegetarian diets: What do we know of their effects on common chronic diseases? American Journal of Clinical Nutrition, 89 (suppl.), 1607s$1612 \mathrm{~s}$.

Guenther, P.M, Dodd, K.W., Reedy, J, \& Krebs-Smith, S.M. (2012). Most Americans eat much less than recommended amounts of fruits and vegetables. Journal of the American Dietetics Association, 106 (9), 1371- 1379.

Guyatt, G.H., Feeny, D.H., \& Patrick, D.L. (1993). Measuring health-related quality of life. Annals of Internal Medicine, 118, 622- 629. 
Hall, J.E., Granger, J.P., Reckelhoff, J.F., \& Sandberg, K. (2008). Hypertension and cardiovascular disease in women. Hypertension, 51, 951.

Hansson, L.M., Galanti, M.R., \& Bergstrom, R. (2000). Factors affecting reproducibility of dietary reports using food frequency questionnaires. European Journal of Clinical Nutrition, 54, 658- 664.

Hasler, C.M. (1998). Functional foods: Their role in disease prevention and health promotion. Food Technology, 52, 63-70.

Hasler, C.M. \& Blumberg, J.B. (1999). Introduction symposium on phytochemicals: Biochemistry and physiology. Journal of Nutrition, 129, 756S- 757S.

“Heart Disease”. Centers for Disease Control and Prevention. n.a. Web. 19 Nov. 2012.

“Health-Related Quality of Life”. Centers for Disease Control. n.a. Web. 23 Oct. 2012.

"Healthy People." Centers for Disease Control and Prevention. n.a. Web. 29 Oct. 2012.

Heber, D. (2004). Vegetables, fruits and phytoestrogens in the prevention of diseases. Journal of Postgraduate Medicine, 50 (2), 145-149.

Hermansen, K. (2000). Diet, blood pressure and hypertension. British Journal of Nutrition, 83 (suppl.), S113-S119.

Hu, F.B. (2003). Plant-based foods and prevention of cardiovascular disease: an overview. American Journal of Clinical Nutrition, 78 (suppl), 544S-551S.

Hung, H., Joshipura, K.J., Jiang, R., Hu, F.B., Hunter, D., Smith-Warner, S.A., Colditz, G. A., Rosner, B., Spiegelman, D., \& Wilett, W.C. (2004). Fruit and vegetable intake and risk of major chronic disease. Journal of the National Cancer Institute, 96(21), 1577-1584.

Jackson, A.S., \& Pollock, M.L. (1978). Generalized equations for predicting body density of men. British Journal of Nutrition, 40, 497- 504.

Jackson, A.S., Pollock, M.L., \& Ward, A. (1980). Generalized equations for predicting body density of women. Medicine and Science in Sports and Exercise, 12, 175182.

John, J.H., Ziebland, S., Yudkin, P., Roe, L.S., Neil, H.A.W. (2002)Effects of fruit and vegetable consumption on plasma antioxidant concentrations and blood pressure: a randomized controlled trial. Retrieved from http://image.thelancet.com/extras/01art9006web.pdf 
Johnston, C.S., Taylor, C.A., \& Hampl, J.S. (2000). More Americans are eating "5 a day" but intakes of dark leafy green and cruciferous vegetables remain low. American Society for Nutritional Sciences. Retrieved from http://jn.nutrition.org/content/130/12/3063.full.pdf+html

Key, T.J., Schatzkin, A., Willett, W.C, Allen, N.E., Spencer, E. A., \& Travis, R.C. (2004). Diet, nutrition and the prevention of cancer. Public Health Nutrition, 7(1A), 187-200.

Kiefer, I., Prock, P., Lawrence, C., Wise, J., Bieger, W., Bayer, P., Rathmanner, T., Kunze, M., \& Rieder, A. (2004). Supplementation with mixed fruit and vegetable juice concentrates increased serum antioxidants and folate in healthy adults. Journal of the American College of Nutrition, 23 (3), 205-211.

Klijs, B., Nusselder, W.J., Looman, C.W., \& Mackenback, J.P. (2011). Contribution of chronic disease to the burden of disability. Plos ONE, 6 (9), 1-8.

Koch, T. (2000). Life quality vs the 'quality of life': Assumptions underlying prospective quality of life instruments in health care planning. Social Science \& Medicine, 51, 419-427.

Koertge, J., Weidner, G, Elliot-Eller, M, Scherwitz, L, Merrit-Worden, Marlin, R....Ornish, D. (2003). Improvement in medical risk factors and quality of life in women and men with coronary artery disease in the Multicenter Lifestyle Demonstration Project. American Journal of Cardiology, 91(11), 1316-1322.

Lampe, J. W. (1999). Health effects of vegetables and fruit: Assessing mechanisms of action in human experimental studies. American Journal of Clinical Nutrition, 70 (suppl.), 475S- 490S.

Lawes, C.M.M., Vander Hoorn, S., Law, M.R., Elliot, P., MacMahon, S., \& Rodgers, A. (2004). High Blood Pressure. In Ezzati, M., Lopez, A.D., Rodgers, A., \& Murray, C.J.L. (Eds.), Comparative Quantification of Health Risks( 281-389). Geneva: World Health Organization.

"Leading Causes of Death." Center for Disease Control and Prevention. n.d. Web. 20 Nov. 2012.

Liu, R.H. (2003). Health benefits of fruit and vegetables are from additive and synergistic combinations of phytochemicals. American journal of Clinical Nutrition, 78(suppl.), 517S- 520S

Liu, S. (2003). Whole-grain foods, dietary fiber, and type 2 diabetes: searching for a kernel of truth. American Journal of Clinical Nutrition, 77, 527- 529.

Liu, S., Manson, J.E., Lee, I., Cole, S.R., Hennekens, C.H., Willett, W.C., \& Buring, J.E. (2000). Fruit and vegetable intake and risk of cardiovascular disease: the Women's Health Study. American Journal of Clinical Nutrition, 72, 922- 928. 
Lock, K., Pomerleau, J., Causer, L. Altmann, D.R., \& McKee, M. (2005). The global burden of disease attributable to low consumption of fruit and vegetables: implications for the global strategy on diet. Bulletin of the World Health Organization, 83(2), 100-108.

Lock, K., Pomerleau, J., Causer, L., \& McKee, M. (2004). Low fruit and vegetable consumption. In Ezzati, M., Lopez, A.D., Rodgers, A., \& Murray, C.J.L. (Eds). Comparative Quantification of Health Risks. (597-728) Geneva: World Health Organization.

Lupton, J.R., \& Turner, N.D. (2003). Dietary fiber and coronary disease: Does the evidence support an association? Current Atherosclerosis Reports, 5, 500-505.

"Metrics: Disability-Adjusted Life Year (DALY)". World Health Organization. Web. 26 Nov. 2012.

Mills, Milton. "What every doctor should know about the role of nutrition in the practice of clinical medicine." Adventist Health with Plant-Based Nutrition Professional Conference. Ambridge Even Center, Portland, OR. 21 Sept. 2012. Lecture.

Nestle, M. (1999). Animal v. plant foods in human diets and health: Is the historical record unequivocal? Proceedings of the Nutrition Society, 58, 211-218.

Nishida, C., Uauy, R., Kumanyika, S., \& Setty, P. (2004). The joint WHO/FAO Expert Consultation on diet, nutrition, and the prevention of chronic diseases: process, products and policy implications. Public Health Nutrition, 7 (1A), 245- 250.

“Noncommunicable Diseases”. World Health Organization. n.a. Web. 23 Nov. 2012.

Ornish, Dean. (2008). Yes, Prevention is Cheaper Than Treatment. Newsweek Magazine. Retrieved from http://www.pmri.org/publications/newsweek/Yes_Prevention_is_Cheaper_than_T reatment_Dean_Ornish.pdf

Ornish, D., Brown, S.E., Scherwitz, L.W., Billings, J.H., Armstrong, W.T., Ports, T.A., McLanahan, S.M., Kirkeeide, R.L., Brand, R.J., \& Gould, K.L. (1990). Can lifestyle changes reverse coronary heart disease? The Lifestyle Heart Trial. The Lancet, 336 (8708), 129-133.

Partnership for Prevention. (2009). The economic argument for disease prevention: Distinguishing between value and savings. Washington DC: Woolf, S.H., Husten, C.G., Lewin, L.S., Marks, J.S., Fielding, J.E., \& Sanchez, E.J.

Perloff, D., Grim, C., Flack, J., Frohlich, E., Hill, M., McDonald, M., \& Mogenstern, B.Z. (1993). Human blood pressure determination by sphygmomanometry. Circulation, 88, 2460- 2470.

"Preventing chronic disease: a vital investment". World Health Organization. n.a. Web. 21 Oct. 2012. 
Roberts, C.K. \& Barnard, R.J. (2005). Effects of exercise and diet on chronic disease. Journal of Applied Physiology, 98, 3-30.

Rowland, I. (1999). Optimal nutrition: Fibre and phytchemicals. Proceedings of the Nutrition Society, 58, 415-419.

Sacks, F.M., Svetkey, L.P., Vollmer, W.M., Appel, L.J., Bray, G.A., Harsha, D., Obarzanek, E., Conlin, P.R., Miller III, E.R., Simons-Morton, D.G., Karanja, N., Lin, P. (2001). Effects on Blood pressure of reduced dietary sodium and the dietary approaches to stop hypertension (DASH) diet. The New England Journal of Medicine, 344 (1), 3-10.

Schreiner, M. \& Huyskens-Keil, S. (2006). Phytochemicals in fruit and vegetables:Health promotion and postharvest elicitors. Critical Reviews in Plant Sciences, 25, 267278.

Shenoy, S.F., Kazaks, A.G., Holt, R. R., Chen, H.J., Winters, B.L., Khoo, C.S., Poston, W.S.C., Haddock, C.K., Reeves, R.S., Foreyt, J.P., Gershwin, M. R., \& Keen, C.L. (2010). The use of commercial vegetable juice as a practical means to increase vegetable intake: a randomized controlled trial. Nutrition Journal, 9 (38), $1-11$.

Siani, A., Cappucio, F.P., Barba, G., Trevisan, M., Farinaro, E., Lacone, R., Russo, O., Mancini, M, \& Strazzullo, P. (2002). The relationship of waist circumference to blood pressure: The Olivetti Heart Study. American Journal of Hypertension, 15 (9), $780-786$.

"State Indicator Report on Fruits and Vegetables 2009". Centers for Disease Control. n.a. Web. 17 Oct. 2012.

Steffen, L.M., Kroenke, C.H., Yu, X., Pereira, M.A., Slattery, M.L., Van Horn, L., Gross, M.D., \& Jacobs Jr., D.R. (2005). Associations of plant food, dairy product, and meat intakes with 15-y incidence of elevated blood pressure in young black and white adults: The Coronary Artery Risk Development in Young Adults (CARDIA) Study. American Journal of Clinical Nutrition, 82, 1169-1177.

Steinmetz, K.A., \& Potter, J.D. (1996). Vegetables, fruit, and cancer prevention: A review. Journal of the American Dietetic Association, 96 (10), 1027- 1039.

Sticher, M.A., Smith, C.B., \& Davidson, S. (2010). Reducing heart disease through the vegetarian diet using primary prevention. Journal of the American Academy of Nurse Practitioners, 22, 134-139.

Storz, G., \& Imlay, J. (1999). Oxidative stress. Current Opinion in Microbiology, 2, 188194. 
Strandberg, T.E., Salomaa, V.V., Vanhanen, H.T., \& Pitkala, K. (2001). Blood pressure and mortality during an up to 32-year follow-up. Journal of Hypertension, 19, $35-$ 39.

Sung, K.C., \& Ryu, S.H. (2004) Insulin resistance, body mass index, waist circumference are independent risk factor for high blood pressure. Clinical and Experimental Hypertension, 26 (6), 547- 556.

Svetkey, L.P., Simons-Morton, D., Vollmer, W.M., Appel, L.J., Conlin, P.R., Ryan, D., Ard, J., \& Kennedy, B.M. (1999). Effects of dietary patterns on blood pressure subgroup analysis of the dietary approaches to stop hypertension (DASH) randomized clinical trial. Archives of Internal Medicine, 159 (3), 285-293.

Szeto, Y.T., Tomlinson, B., \& Benzie, I.F.F. (2002). Total antioxidant and ascorbic acid content of fresh fruits and vegetables: Implications for dietary planning and food preservation. British Journal of Nutrition, 87, 55-59.

Tang, G., Qin, J., Dolnikowski, G.G., Russell, R.M., \& Grusak, M.A. (2005). Spinach or carrots can supply significant amounts of vitamin A as assessed by feeding with intrinsically deuterated vegetables. American Journal of Clinical Nutrition, $82,{ }^{\wedge} 821-828$.

Taylor, C.A., Hampl, J.S., \& Johnston, C.S. (2000). Low intakes of vegetables and fruits, especially citrus fruits, lead to inadequate vitamin $\mathrm{C}$ intakes among adults. European Journal of Clinical Nutrition, 54, 573-578.

Terry, P., Giovannucci, E., Michels, K.B., Bergkvist, L., Hansen, H., \& Holmberg, L. (2001). Fruit, vegetables, dietary fiber, and risk of colocrectal cancer. Journal of the National Cancer Institute, 93, 525-533.

Theuwissen, E., \& Mensink, R.P. (2008). Water-soluble dietary fibers and cardiovascular disease. Physiology and Behavior, 94, 285- 292.

“Top 10 Causes of Death.” World Health Organization. n.a. Web. 20 Nov. 2012.

Tucker, K.L. (2004). Dietary intake and coronary heart disease: A variety of nutrients and phytochemicals are important. Prevention, 6, 291- 302.

Wang, H., Cao, G., \& Prior, R.L. (1996). Total antioxidant capacity of fruits. Journal of Agriculture: Food Chemistry, 44, 701- 705.

"What is Heart Disease?.” American Heart Association. Web. 19 Nov. 2012.

Whitney, E., \& Rolfes, S.R. (2008). Understanding Nutrition. Ohio: Cengage Learning,. Print.

WHOQOL: Measuring Quality of Life. n.a. Geneva: World Health Organization, 1997. 
"WMA Statement on the global burden of chronic disease." World Medical Journal. n.a. Oct 2011. Web. 24 Oct. 2012.

Young, I.S., \& Woodside, J.V. (2001). Antioxidants in health and disease. Journal of Clinical Pathology, 54, 176- 186.

Zhang, X., Shu, X., Xiang, Y., Yang, G., Li, H., Gao, J., Cai, H., Gao, Y., \& Zheng, W. (2011). Cruciferous vegetable consumption is associated with a reduced risk of total and cardiovascular disease mortality. American Journal of Clinical Nutrition, 94, 240-246. 


\section{Appendix A: Informed Consent Form}

You are invited to participate in a study on the benefits of increased fruit and vegetable intake on the daily diet titled 'Effect of Green Smoothie Consumption on Blood Pressure and Health-Related Quality of Life: A Randomized Controlled Trial'. 'Randomized Controlled Trial' merely implies that volunteers for the study will not be allowed to choose which group to be a part of (the control or experiment group), but instead will be chosen at random to reduce the likeliness of a weighted outcome in results. This study is a thesis project conducted by Emiko Maeda, the principal investigator, which concludes the final steps towards her Master's Degree in Health Studies at Portland State University (School of Community Health; Advisor- Gary Brodowicz, Ph.D.).

The purpose of this study is to investigate the perceived health benefits of supplementing the daily diet with a blended drink of fruits and leafy greens for four consecutive weeks. Interested volunteers must meet qualifying criteria such as being over the age of 18 , in good health, capable of transporting themselves to a designated location for the data collection when needed for scheduled meetings (2-3 meeting total), and be financially capable to purchase produce for the smoothies and also have time to make the beverage on a daily basis. Again, it is the sole responsibility of volunteers to purchase the ingredients for the smoothies and make them for themselves each day. Furthermore, volunteers must be willing to accept the possibility of being randomly selected for either group (experiment or control), meaning that although physical data will be collected from all volunteers at two different times, participants have a 50/50 chance of being placed in the group drinking the smoothies or the group that will do nothing more than have their data collected at two separate times. Volunteers may withdraw from the study at any time and for any reason.

Methods: All participants will have resting blood pressure, height, weight, body circumference and skinfold thickness measurements taken before and after the 4-week experimental period. Resting blood pressure is the measurement of the pressure of circulating blood against the walls of the blood vessels inside the body. Skinfold thickness is a commonly used method of measuring the percentage of body fat in human subjects and is done by measuring the amount of skin that can be pinched by an instrument called 'skinfold calipers'. The resulting numbers are entered into a scientific formula for a final number demonstrating the percentage of body fat of that individual. The study also includes filling out the Nutritional Assessment Questionnaire, A 316 question health survey regarding the severity and frequency of symptoms related to health, both before and after the 4-week period. However, those placed into the experimental group will have the additional responsibilities of buying the ingredients for and making green smoothies daily as outlined in a detailed schedule for the 4 weeks. The 'schedule' will be a strict outline of ingredients and portions to assure that all participants are drinking the same smoothies throughout the study. Prior to the beginning of the study, the experiment participants (volunteers drinking smoothies) will be taught how to make green smoothies and offered ample support for questions or concerns about the 
study. It is the responsibility of the volunteers to purchase ingredients for and making the smoothies according to the schedule.

Participants should expect to spend roughly one hour for each session at the chosen location for data collection both at the beginning and end of the study. Filling out the Nutritional Assessment Survey will vary for individuals, but likely take no longer than 20 minutes each.

Benefits: The participants of the study will gain the benefit of knowing they have added to the body of knowledge pertaining to nutritional therapy. All participants also receive personal health information pertaining to body circumference and skinfold measurements, estimated fat percentage and resting blood pressure. Furthermore, intervention participants will have the added benefit of increasing daily raw fruit and vegetable consumption that offers various health benefits including reduced risks for several chronic diseases and increased levels of hydration and fiber intake.

Risks and Safeguards: Any change in diet or daily habit incurs the risk of emotional or physical discomfort. Participation in this study may require volunteers to alter eating habits that may cause temporary gastrointestinal discomfort if unaccustomed to large amounts of raw fruits and vegetables. Participants will be encouraged to continue with the study but scale back green smoothie intake a few days or a lesser amount if discomfort is severe.

You agree that participation in this study represents your own personal choice to volunteer in the nutritional therapy study 'The Effects of Green Smoothies on Blood Pressure and Quality of Life'.

You understand that as a volunteer in this study you may be asked and expected to adhere to the scheduled regimen of green smoothies for a 4-week period which may be drastically different from your normal routine. This study may require you to grocery shop every few days to ensure freshness and prepare smoothies daily.

By signing this consent form you agree to be in good health and free from complications that may otherwise impede you from participating in this nutritional therapy study. All participants are required to fill out an additional health questionnaire (Santa Ana Health Questionnaire) in order to discern other health risks prohibiting participation in this study that will be reviewed by a medical professional to ensure eligibility into the volunteer pool.

Every effort will be made to uphold confidentiality of your identity and collected data. Once admitted into the study, each participant will be assigned a 5-digit number; all data will be coded under this number so that the research team will be unaware of your true identity. By signing this form you are permitting Emiko Maeda to analyze, print and publish descriptive characteristics and data from your participation without the risk of identification. However, due to group style meetings, your participation will not be 
anonymous to others participating in the study, although all collected data will be kept confidential.

All collected data will be kept confidential inside the research team and kept as the sole property of Emiko Maeda and her Advisor, Gary Brodowicz, for no fewer than three calendar years from the termination of the study. All original documentation will be stored in the safety of the home of the principal investigator and the locked office of Gary Brodowicz. Results of the study and resulting Master's Thesis manuscript will be available to you if you so desire.

Your signature below certifies that you are over the age of 18, you understand the risks and benefits involved in participation of this study, you are of sound mind and body, and are choosing to participate by your own free will. All participants will receive copies of this Consent form.

Signature: Date:

Contact Information:

Emiko Maeda, Principal Investigator

Emiko7@gmail.com

503.703.2544

Gary Brodowicz, Ph.D., Advisor

Portland State University, School of Community Health

brodowiczg@pdx.edu

503.725.5119

Human Subjects Research Review Committee

Office of Research and Sponsored Projects

600 Unitus Building

Portland State University

503.725.4288

1.877 .480 .4400 


\section{Appendix B: Data Sheet}

ID \#

OBSERVATION 1 or 2 (circle one)

AGE

GENDER

HEIGHT

1. WEIGHT

2. WEIGHT

AVG.

ETHNICITY: WHITE

ASIAN _ _ HISPANIC

OTHER

\section{BLOOD PRESSURE}

1. SYSTOLIC

DIASTOLIC

2. SYSTOLIC

DIASTOLIC

AVG.: SYSTOLIC

DIASTOLIC

RESTING H.R.

RESTING H.R.

H.R. AVG.

BODY MEASUREMENTS

1.

1.

1.

AVG.

2.

2.

2.

AVG.

3.

3.

3.

AVG.

$\%$ BODY FAT

WAIST: 1.

2.

AVG.

HIP: 1.

2.

AVG.

WAIST/HIP 


\section{Appendix C: Nutritional Assessment Questionnaire}

Return to: National Integrased Health Associates

Name:

Birthdate
Date:

Gender

Please list your five major health concerns in order of importance:

1.

2.

3

5.

PART I

Read the following questions and fill in the number that applies:

KEY: $\quad 0$ (or leave blank) $=$ Do not consume or use $\quad 2=$ Consume or use weekly

1 = Consume or use 2-3 times/month 3 = Consume or use daily

DIET

1. Alcohol

Artificial sweeteners

Candy or other sweets

Carbonated beverages

Cheraing tobacco

_Cigars/pipes

\section{LIFESTYLE}

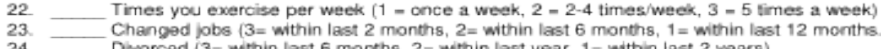

MEDICATIONS

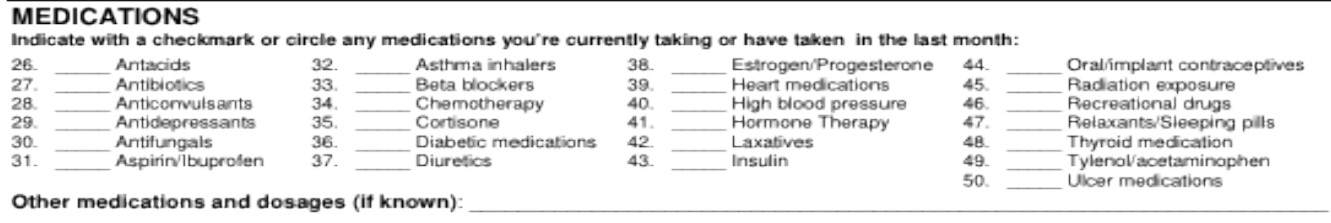

Other medications and dosages (if known

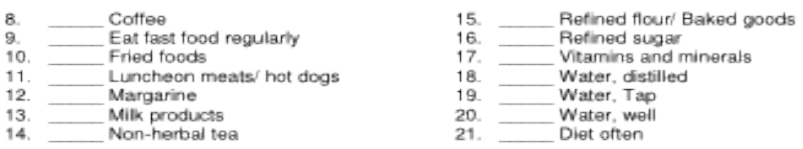

PART II

Read the following questions and fill in the number that applies:

(How significant is the symptom? How true is the statement? 0 means not at all, 3 means extremely true.

KEY : 0 (or leave blank) $=$ No or Do not have the symptom, the symptom does not occur $1=$ Yes or It is a minor or mild symptom or it rarely occurs (once a month or less) 2- It is a moderate symptom or it occasionally occurs (weekly)

Please Answer Each Section Carefully

They relate to different body systems.
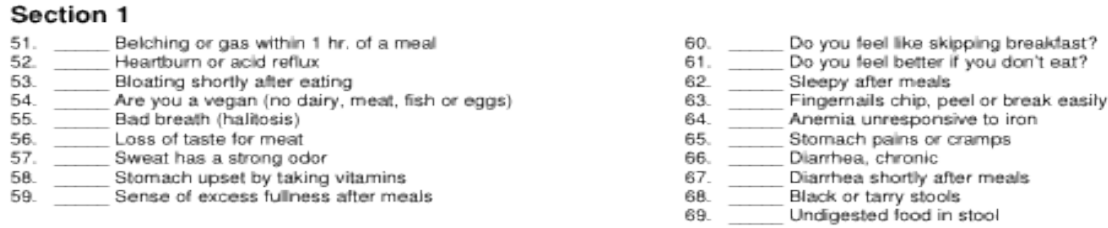


\section{Nutritional Assessment Questionnaire}

\begin{tabular}{|c|c|}
\hline \multicolumn{2}{|c|}{ Section 2} \\
\hline 70. & Pain between shoulder blades \\
\hline 71. & Stomach upset by greasy foods \\
\hline 72. & Greasy or shiny stools \\
\hline 73. & Nausea \\
\hline 74. & Sea, car or airplane sickness, motion sickness \\
\hline 75. & History of morning sickness ( 1 - yes, 0 - no) \\
\hline 76. & Light or clay colored stools \\
\hline 77. & Dry sivin, itchy feet andior skin peets on feet \\
\hline 78. & Headache over the eye \\
\hline 79. & Gallbladder attacks (past or present) \\
\hline 80. & Gallbladder removed ( 1 - yes, $0=$ no) \\
\hline 81. & Bitter taste in mouth, especially after meals \\
\hline 82. & Become sick if drinking wine \\
\hline 83. & If drinking alcohol, easity intoxicated \\
\hline
\end{tabular}

\section{Section 3}

\begin{tabular}{|c|c|}
\hline 98. & Food allergies \\
\hline 99. & Abdominal bloating 1 to 2 hours after eating \\
\hline 100. & Specific foods make you tired or bloated (1- yes, $0-$ no) \\
\hline 101. & Pulse speeds after eating \\
\hline 102. & Airborne alergies \\
\hline 103. & Experience hives \\
\hline 104. & Sinus congestion, "stuffy head" \\
\hline 105. & Crave bread or noodles \\
\hline 106. & Alternating constipation and diarrhes \\
\hline
\end{tabular}

106. Altemating constipation and diarthes

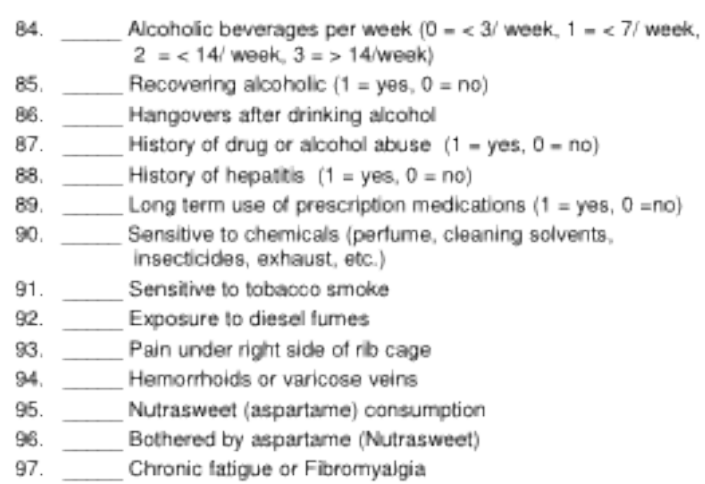

\begin{tabular}{|c|c|}
\hline 107. & Crohn's disease $(1=$ yes, $0=n o)$ \\
\hline 108. & Wheat or grain sensitivity \\
\hline 109. & Dairy sensitivity \\
\hline 110. & Are there loods you could not give up ( $1-$ yes, $0=$ no) \\
\hline 11. & Asthma, sinus intections, stuffy nose \\
\hline 112. & Blzarre vivid or nightmarish dreams \\
\hline 13. & Use over-the-counter pain medications \\
\hline & Feel spacey or unreal \\
\hline
\end{tabular}

\section{Section 4}

115. Anus itches

116. Coated tongue

117. Feel worse in moldy or musty place

118. Taken any antibiotic for a combined time of $(1=<1 \mathrm{mo}, 2=<3$ mos., $3=>3$ mos. $)$

119. Fungus or yeast infections

120. _ Ping worm, "jock illch", "athletes foot", nail fungus

121. Eating sugar, starch or drinking alcohol increases yeast symptoms

122. Stools hard or difficult to pass

123. ___ History of parashes $(1=$ yes, $0=$ no)

\begin{tabular}{|c|c|}
\hline 124. & Less than one bowel movement per day \\
\hline 125. & Stools have corners or edges are flat or ribbon shaped \\
\hline 126. & Stools are not well formed (loose) \\
\hline 127. & Inritable bowel or mucus colitis \\
\hline 128. & Blood in stool \\
\hline 129. & Mucus in stool \\
\hline 130. & Excessive foul smelling lower bowel gas \\
\hline 131. & Bad breath or strong body odors \\
\hline 132. & Painful to press along outer sides of thighs (Tliotbial Band) \\
\hline 133. & Cramping in lower abdominal region \\
\hline 134. & Dark circles under eyes \\
\hline
\end{tabular}

\section{Section 5}

135. History of Carpal Tunnel Syndrome (1 - yes, 0 - no)

136. History of lower right abdominal pain $(1=y e s, 0=n 0)$

137. History of stress tractures

138. ___ Bone loss (reduced density on bone scan)

139. Are you shorter than you used to be? $(1-$ yes, $0=$ no)

140. Calf, loot or toe cramps at rest

141. Cold sores, lever blisters or herpes lesions

142. Frequent fevers

143. ___ Frequert skin rashes and / or hives

144. Have you ever had a hemiated disc? $(1=$ yes, $0=$ no $)$

145. Excessively flexible joints, "double jointed"

146. J__ Joints pop or click

147. Pain or swelling in joints

148. ___ Bursitis or tendonitis

149. History of bone spurs $(1=$ yes, $0=n o)$

\begin{tabular}{|c|c|}
\hline 150. & Morning stiffness \\
\hline 151. & Vomiting or nausea \\
\hline 152. & Crave chocolate \\
\hline 153. & Feet have a strong odor \\
\hline 154. & Tendency to anemia \\
\hline 155. & Whtes of eyes (sclera) blue tinted \\
\hline 156. & Hoarseness \\
\hline 157. & Difficulty swallowing \\
\hline 158. & Lump in throat \\
\hline 159. & Dry mouth, eyes and / or nose \\
\hline 160. & Gag easily \\
\hline 161. & White spots on fingernails \\
\hline 162. & Cuts heal slowly and / or scar easily \\
\hline 163. & Decreased sense of taste or smell \\
\hline
\end{tabular}

2 - Moderate symptom, occurs occasionally (weekly) 3 - Severe symplom, frequently occurs (daily) 
Nutritional Assessment Questionnaire

\begin{tabular}{|c|c|}
\hline \multicolumn{2}{|c|}{ Section 6} \\
\hline 164. & Aspirin is an effective pain reliever ( $1-$ yes, $0=$ no) \\
\hline 165. & Crave fatty or greasy loods \\
\hline 166. & Low or reduced tat diet (past or present) \\
\hline 167. & Tension headaches at base of skull \\
\hline
\end{tabular}

\section{Section 7}

172. Awaken a few hours after falling asleep, hard to get back to sleep

173. ___ Crave sweets

174. —_ Eat desserts or sugary snacks

175. __ Binge or uncontrolled eating

176. Excessive appetite

177. Crave coffee or sugar in the afternoon

178. __ Sleapy in afternoon

\section{Section 8}

\begin{tabular}{|c|c|}
\hline 186. & Muscles become easily fatigued \\
\hline 187. & Feel worse, sore after moderate exercise \\
\hline 188. & Vuinerable to insect bites \\
\hline 189. & Lass of muscle tone, heaviness in ams / legs \\
\hline 190. & Enlarged heart, or heart failure \\
\hline 191. & Pulse siow / below $65(1=$ yes, $0=$ no $)$ \\
\hline 192. & Ringing in the ears / Tinnitus \\
\hline 193. & Numbness, tingling or itching in extremities \\
\hline 194. & Depressed \\
\hline 195. & Fear of impending doom \\
\hline 196. & Worrier, apprehensive, anxious \\
\hline 197. & Nervous or agitated \\
\hline 198. & Feeings of insecurity \\
\hline 199. & Heart races \\
\hline
\end{tabular}

\section{Section 9}

\begin{tabular}{|c|c|}
\hline 214. & Tend to be a "night person" \\
\hline 215. & Difficulty falling asleep \\
\hline 216. & Slow starter in the morring \\
\hline 217. & Keyed up, trouble calming down \\
\hline 218. & High blood pressure (normal 120/80) \\
\hline 219. & Headache after exercising \\
\hline & Feeling wired or jittery if drinking cottee \\
\hline 221. & Clench or grind teeth \\
\hline 222. & Calm on the outside, troubled inside \\
\hline 223. & Chronic low back pain, worse with fatigue \\
\hline 224. & Become dizzy when standing up suddenly \\
\hline 225. & Ditficult maintaining manipulative correction \\
\hline & Pain after manipulative correction \\
\hline
\end{tabular}

226. ___ Pain after manipulative correction

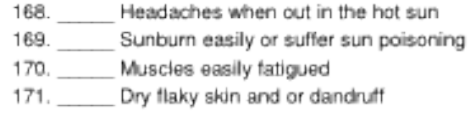

\section{Section 10}

240. Over $6^{\prime} 6^{\circ}$ tall (Mature height)

241. Early sexual deveiopment (before age 10$)(1-$ yes, 0 - no)

242. Increased libido

243. Spltting type headache

244. Memory faling

245. Ability to tolerate sugar

\begin{tabular}{|c|c|}
\hline Do. & Can hear heart beat on pillow at night \\
\hline 201. & Whole body or limb jerk as falling asleep \\
\hline 202. & Night sweats \\
\hline 203. & Restless leg syndrame \\
\hline 204. & Cheilosis (cracks at comer of mouth) \\
\hline 205. & Fragile skin, easily chaffed, as in shaving \\
\hline 206. & Polyps or warts \\
\hline 207. & MSG sensitivity \\
\hline 208. & Wake up without remembering dreams \\
\hline 209. & Take birth control pills \\
\hline 210. & Small bumpe on back of arms \\
\hline 211. & Strong light at night irritates eyes \\
\hline 212. & Nose bleeds and / or tend to bruise easily \\
\hline 213. & Bleeding gums especially when brushing te \\
\hline
\end{tabular}

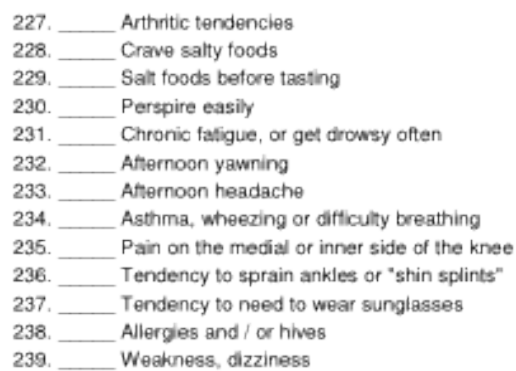

\begin{tabular}{|c|c|}
\hline 246. & Under $4^{\prime} 10^{*}$ (Mature height) \\
\hline 247. & Decreased Ibido \\
\hline 248. & Abnormal thirst \\
\hline 249. & Weight gain around hips or waist \\
\hline 250. & Menstrual disorders \\
\hline 251. & Deiayed (after age 13) sexual development $(1-$ yes, $0-$ no) \\
\hline 252. & Tendency to ulcers or colitis \\
\hline
\end{tabular}

Key: 0 (or leave blank) - No or Do not have symplom, symptom does not cocur 2 - Moderate symptom, occurs occasionally (weekly) $1=$ Yes or Minor or mild symptom (once a manth or less) 


\section{Nutritional Assessment Questionnaire}

\begin{tabular}{|c|c|}
\hline \multicolumn{2}{|c|}{ Section 11} \\
\hline 253. & Allergic to iodine \\
\hline 254. & Difficulty gaining weight, even with large appefite \\
\hline 255. & Nervous, emotional, cant work under pressure \\
\hline 256. & Inward trembling \\
\hline 257. & Flush easily \\
\hline 258. & Fast puise at rest \\
\hline 259 . & Intolerance to high lemperatures \\
\hline 260. & Difficuty losing weight \\
\hline
\end{tabular}

\section{Section 12 - Men Only}

269.___ Prostate problems
270._ Urination difficult or dribbling
271 Difficut to start and stop urine stream
272 __ Pain or buming with unination

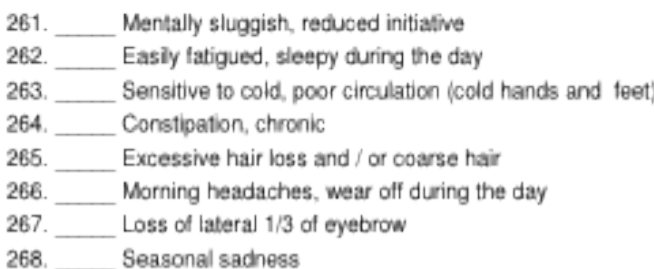

273. Waking to urinate at night
274 In___ Interruption of stream during urination
$275 . \quad$ Pain on inside of legs or heals
$276 . \quad$ Feeling of incomplete bowel evacuation

$277 . \quad$ Decreased sexual function

\begin{tabular}{|c|c|}
\hline \multicolumn{2}{|c|}{ Section 13 - Women Only } \\
\hline 278. & Depression during periods \\
\hline 279. & Mood swings associated with periods (PMS) \\
\hline 280. & Crave chocolate around periods \\
\hline 281. & Breast tendemess associated with cycle \\
\hline 282. & Excessive menstrual flow \\
\hline 283. & Scarty blood flow during periods \\
\hline 284. & Oocasional skipped periods \\
\hline 285. & Variations in menstrual cycles \\
\hline 286. & Endometriosis \\
\hline 287. & Uterine flbroids \\
\hline
\end{tabular}

\begin{tabular}{|c|c|}
\hline 288. & Breast fibroids, benign masses \\
\hline 289. & Painful intercourse (dyspareunia) \\
\hline 290. & Vaginal discharge \\
\hline 81. & Vaginal dryness \\
\hline 82. & Vaginal itchiness \\
\hline 93. & Gain weight around hips, thighs and buttocks \\
\hline 94. & Excess facial or body hair \\
\hline & Hot flashes \\
\hline & Night sweats (in menopausal fernales) \\
\hline & Thiming skin \\
\hline
\end{tabular}

297. Thimning skin

\section{Section 14}

298. Aware of heavy and / or irregular breathing
299. Discomfort at high altitudes
300. - Air hunger" and / or yawn trequently
301.__ Compelled to open windows in a closed room
302. Shortness of breath with moderate exartion

303. Anikles swell, especially at end of day
304. Cough at night
305. Blush or tace tums red for no reason
306. Dul pain or tightness in chest and / or radiate into night am,
307. Morse with exertion

\section{Section 15}

308. Pain in mid back region
309.___ Dark circles under eyes and / or putty eyes
310. History of kidney stones $(1=$ yes, $0=$ no $)$

\section{Section 16}

\begin{tabular}{|c|c|}
\hline 313. & Runny or drippy nose \\
\hline 314. & Calch colds at the beginning of winter \\
\hline 315. & Mucus producing cough \\
\hline 316. & $\begin{array}{l}\text { Frequent infections (ear, sinus, lung. skin, bladder, } \\
\text { kidney, etc.) }\end{array}$ \\
\hline 317. & Frequent colds or flu \\
\hline & $\begin{array}{l}\text { Never get sick ( } 3 \text { - not in last } 7 \text { yrs, } 2=\text { not in last } 4 \text { yrs., } \\
1=\text { not in last } 2 \text { yrs.) }\end{array}$ \\
\hline
\end{tabular}

\section{Cloudy, bloody or darkened urine \\ 312. ___ Unine has a strong odor}




\section{Appendix D: Santa Ana Health Questionnaire}

(Staff Initials: Date:

Santa Ana Health Screening Questionnaire

All information will be kept confidential

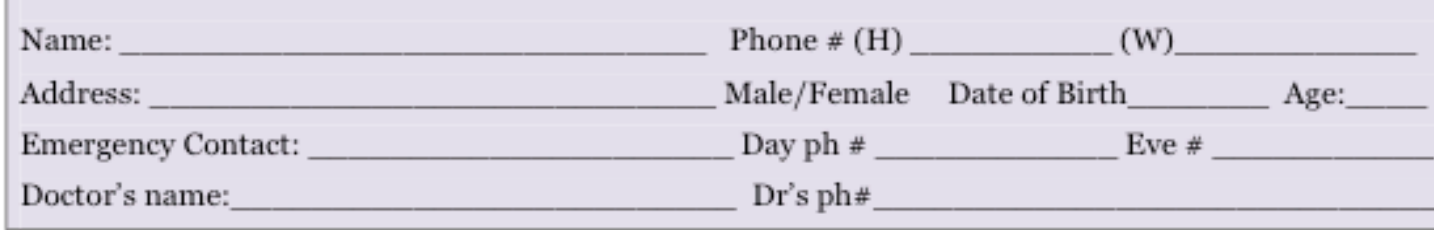

Where do you go for your health care? Santa Ana Clinic Other:

Are you (Circle all that apply): Tribal member Employee Native American visitor

Do you have or have you had any of the following health conditions? (mark all that apply)

HEALTH HISTORY

a heart attack

heart surgery

stroke

pacemaker-implantable cardiac

other heart conditions

\section{HEALTH STATUS}

Diabetes:

If yes, when were you diagnosed?

Asthma or other lung disease

Currently Pregnant

\section{SYMPTOMS}

You experience chest discomfort with exertion

You experience unreasonable breathlessness

You experience dizziness, fainting, or blackouts

You have burning or cramping sensation in you lower legs when walking a short distances

\section{CARDIOVASCULAR RISK FACTORS}

You are a man older than 45 years

You are a woman older than 55 years,

You are a woman who has had a hysterectomy $\mathbf{O R}$ are postmenopausal

Pre-diabetes

You smoke, or quit smoking within the previous 6 months

Your doctor has told you that you have high blood pressure $\mathbf{v}$

You take blood pressure medication

Your doctor has told you that you have high cholesterol

You have a close blood relative who had a heart attack or heart surgery before age 55

(father or brother) or age 65 (mother or sister)

\section{OTHER HEALTH ISSUES}

Given birth in the last 8 weeks: If yes, are you cleared for exercise? ___ Yes __ No Significant recent changes in health status that has not been evaluated by a physician? If yes, please explain:

\section{I do not have any of the above Health conditions or Risk factors}


If you answered yes to diabetes, do you have?

Numbness or loss of feeling in your hands or feet

Vision problem

Diabetic kidney problems

Have you ever had symptoms of high/low blood sugar?

If yes to any of the above explain the best you can:

\begin{tabular}{lc}
\hline List medications: & I am not currently taking any medication. \\
Name_ & Purpose \\
\hline & Purpose \\
\hline & Purpose_ Purpose
\end{tabular}

\section{WAIVER OF LIABILITY}

I have read the questions on this questionnaire, understand them, and have answered them to the best of my knowledge. I understand that, depending on my health and fitness, participation in an exercise program may involve some risks to my health, including injury, abnormal blood pressure response, fainting, dizziness, irregular heart rhythm, and in rare instances, heart attack, stroke or even death. I understand that a medical clearance may be required prior to my participation. I understand that I should report any unusual symptoms that I experience during or after exercise, and that I should update my health history questionnaire should I become aware of a change in my health status. Any questions that I had were answered to my satisfaction. I hereby release the Pueblo of Santa Ana and it's tribal administration, employees, agents, or volunteer staff from any liability for any injuries, which may arise as a result of my participation in an exercise program.

Participant Signature Print name

Date:

\section{Fitness Staff only Interview Questions \& Measurements}

Do you have muscle and/or joint problems that limit your physical activity? _ Yes __No If yes explain:

If yes, have them complete a Pain Assessment

Is Medical Clearance indicated by the Pain Assessment? __ Yes __ No

Below enter how the participant wants to be contacted: W 1 Kathy contacts, 8 2. Participant contacts, N3 not interested Do you know your cholesterol level? __ Yes __ No

If no, would you like to participate in a cholesterol screening? __ Yes _ No Contact \# Have you ever had diabetes screening? __ Yes __No

If no, would you like to participate in a diabetes screening? __ Yes _ No Contact \# If diabetic we recommend that you attend an education session on foot care and blood sugar monitoring with exercise. Are you interested? __ Yes _ No _ _ Contact \#
Blood Pressure: ___ If results is $>140 / 90$, repeat 2 additional separate dates:
1. Date
Results
2. Date Results

Weight (Optional):

$$
\text { Orientation Date Completed: Cardio ___ Strength training }
$$

\section{Staff Coordinator Only}

$B P$ results copied for participant to take to $M D$

Medical Clearance sent to MD

Exercise Clearance received

Contact info given to participant for cholesterol/DM screen

Cholesterol results received \& risk re-evaluated

\begin{tabular}{|c|c|c|c|}
\hline \multicolumn{3}{|c|}{ Clinic Referral } & Walk in \\
\hline NA & Yes & Initals & Date_ \\
\hline NA & Yes & Initals & Date \\
\hline $\mathrm{NA}$ & Yes & Initals & Date \\
\hline NA & Yes & Initals_ & Date_ \\
\hline $\mathrm{NA}$ & Yes & Initals & Date \\
\hline
\end{tabular}
Date

\section{Staff Initials}



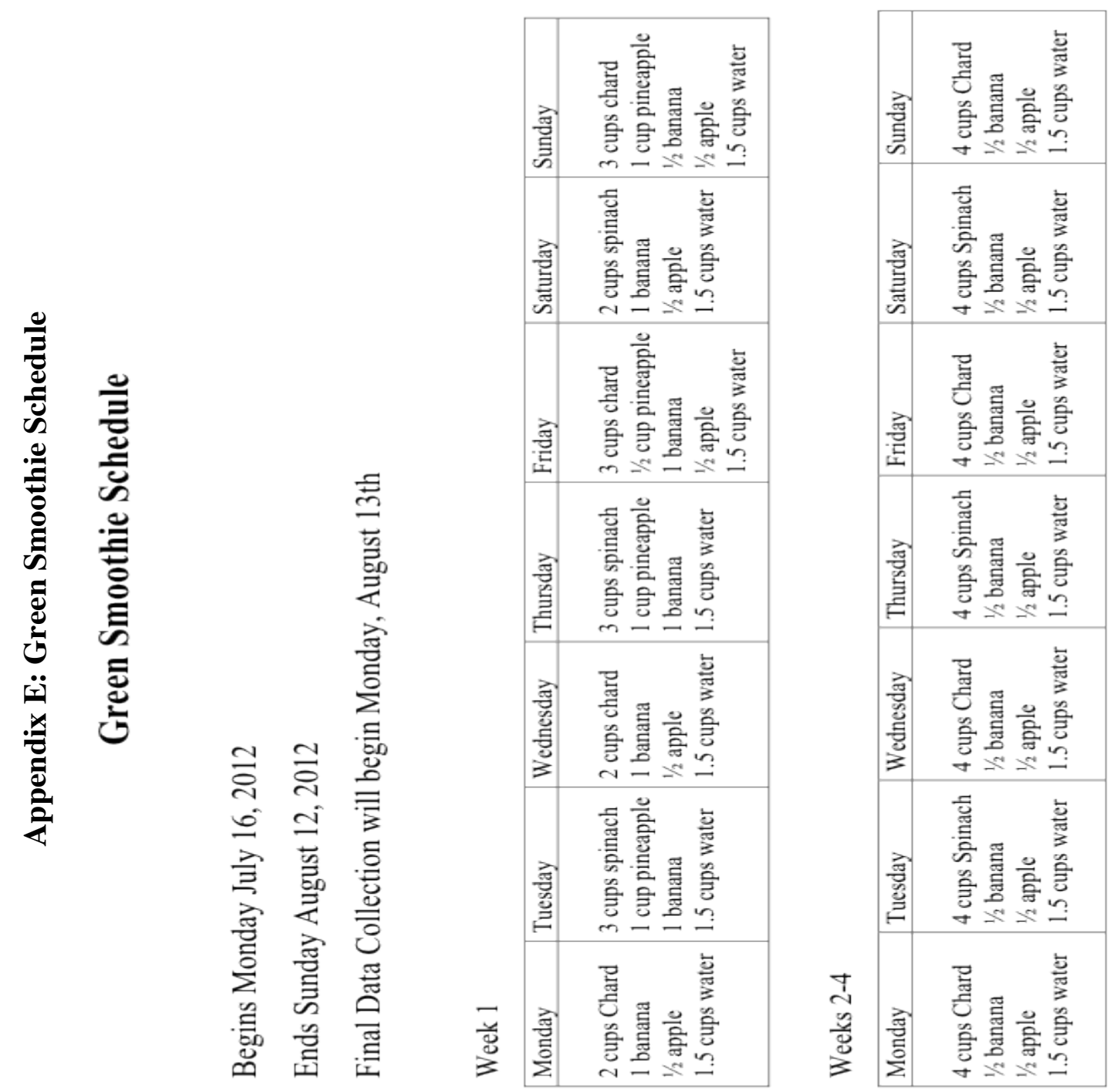


\section{Appendix F: Smoothie Directions}

\section{Directions:}

Wash and chop greens to small pieces and place them in blender with water. Blend until fairly smooth. For best results, peel skin from banana and apple and add them to the blender. Pineapple should be peeled and cored (coring is optional). Blend till desired consistency. Additional water can be added for a thinner consistency, and ice can be added if desired*.

* If dividing the batch made two drinking times, it is recommended to bottle half first, then add ice to the remaining in the blender, blend, then drink. Too far diluted green smoothies $=$ not so tasty.

Things to consider:

- All recipes make approximately 2 pints, the amount you need to drink each day

- The amount of water is approximate and more can be added for a thinner consistency

- Ice can be added if you want to make it colder or more shake-like. However, please make sure you still drink everything made and not dump out the extra after '2 pints' (the ice dilutes it, obviously)

- Although it is not necessary, I find it helpful to have the smoothies first thing in the morning. Not only is it a tasty way to start the day, it is also nice to 'get it out of the way'. I've also had difficulty getting myself to make them if I've been eating food all day

- DO NOT add any other ingredients aside from what is in the recipe aside from ice or more water. I need all participants to be drinking the exact same smoothies so I can compare the effects across the group.

- If you skip a day, I urge you to double up the next day. This is a relatively short study and I am not asking you to change anything else in your life so even skipping a few days throughout the study could be detrimental to the results of this study.

- Organic is preferable but of course not necessary. Especially since ingesting these foods raw, the more pure they are the better they will be for you.

- Please wash all your produce! My motto is: It's either sh*t or chemicals, so wash it.

Tips:

-For Chard it is best to remove the leaves from the stems. The stalks can be hard and may not blend well.

-Although it is recommended to make each batch fresh and drink both pints consecutively, it is ok to split it up. However, if one batch is made and half saved for later, please seal properly and refrigerate for freshness. Fresh juices like these lose their potency quickly and will spoil without proper refrigeration. 


\section{Appendix G: Flyer for Volunteer Recruitment}

\section{NUTRITIONAL THERAPY STUDY VOLUNTEERS NEEDED}

Participants needed for a local research study looking at the effects of increased fruit and vegetable consumption on blood pressure and health quality of life.

The gist of the study: The study is titled 'The Effects of Green Smoothie Consumption on Blood Pressure and Health-Related Quality of Life: A Randomized Controlled Trial'. This study involves adding a homemade smoothie to your daily routine each morning for 4 consecutive weeks. This is a non-funded study, so volunteers will be in charge of purchasing the ingredients and making the smoothies every day. The study should cost each participant around 15-20 dollars a week.

\section{What's in it for you?}

- Two sessions of blood pressure measurement (pre and post study)

- Two sessions of weight, body circumference, and body fat percentage taken by a licensed personal trainer (pre and post)

- An opportunity to increase your fruit and vegetable intake and add to the body of knowledge on nutritional therapy methods

- Participants who finish out the study will be entered to win prizes including

○ \$50 worth of tokens at the Portland Farmers Market

- CSA delivery

- Gift Certificate to Whole Foods Market

\section{Eligibility Criteria:}

○ Must be in good health (free from conditions that may otherwise hinder your ability to participate in the study)

- Daily access to a blender

- Access to and financial ability to purchase and make smoothies daily

- Available for data collection before and after study (week of July 2nd and week of August $13^{\text {th }}$. Sessions are weeknights and weekend days)

\section{You are excluded from the study if:}

- Pregnant, breastfeeding, or intend on getting pregnant during the course of the study

- Have allergies to chard, spinach, lettuce, apples, pineapples or bananas

- Have low blood pressure or are taking medication to control high blood pressure

- Have intentions to drastically change your exercise regimen or diet during the course of this study (aside from the green smoothies of course)

All interested individuals please contact me at emiko7@gmail.com for further details. Hoping to gather $60+$ volunteers so please pass on this information or contact info for friends, family and acquaintances. Please feel free to email with questions and concerns. Thank you, Emiko_emiko7@gmail.com 


\section{Appendix H: NAQ Subsection Pre-Post Difference Scores}

\section{Comparison of Groups}

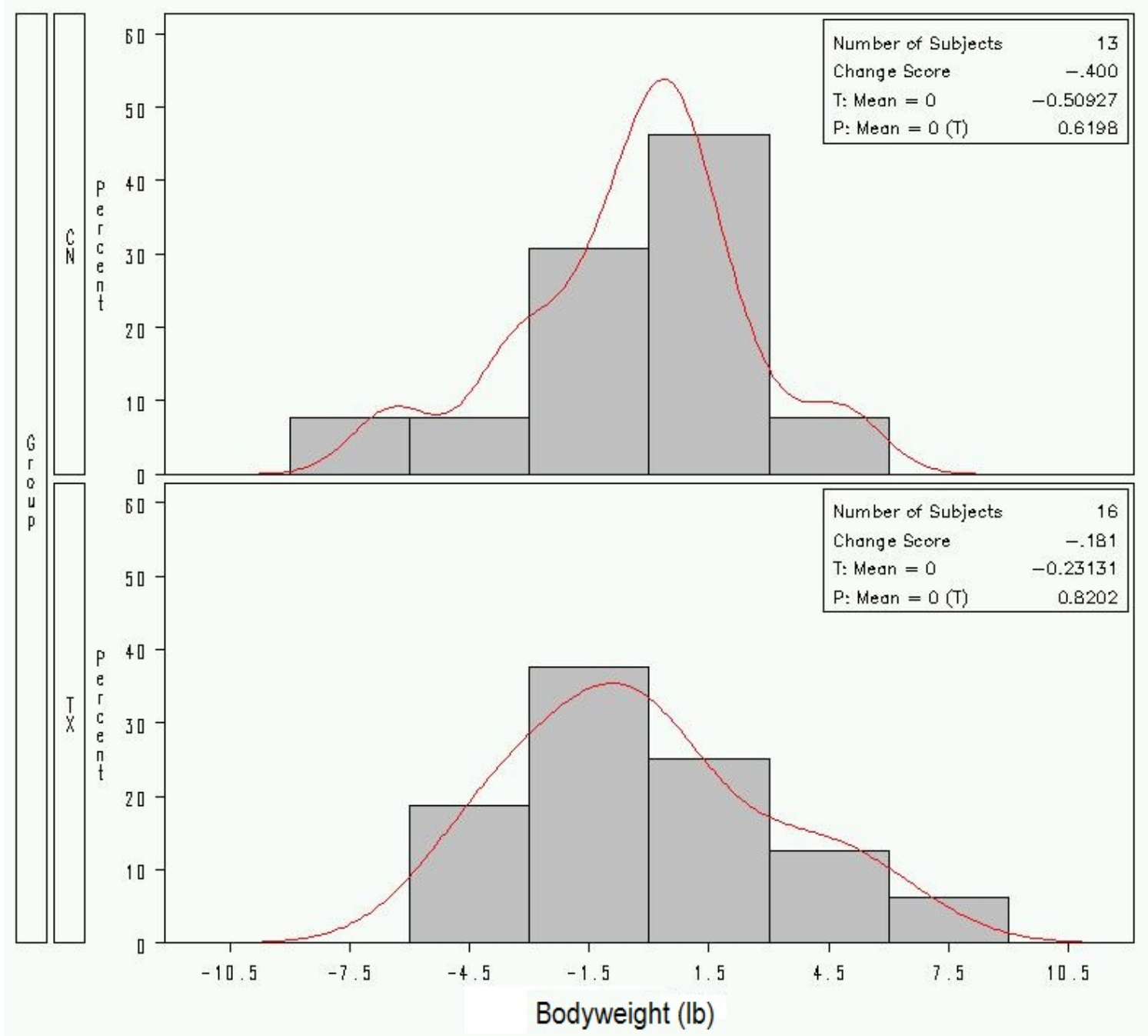

Figure 7. Comparison of Control and Treatment Group Weight Pre-Post Difference Scores 


\section{Comparison of Groups}

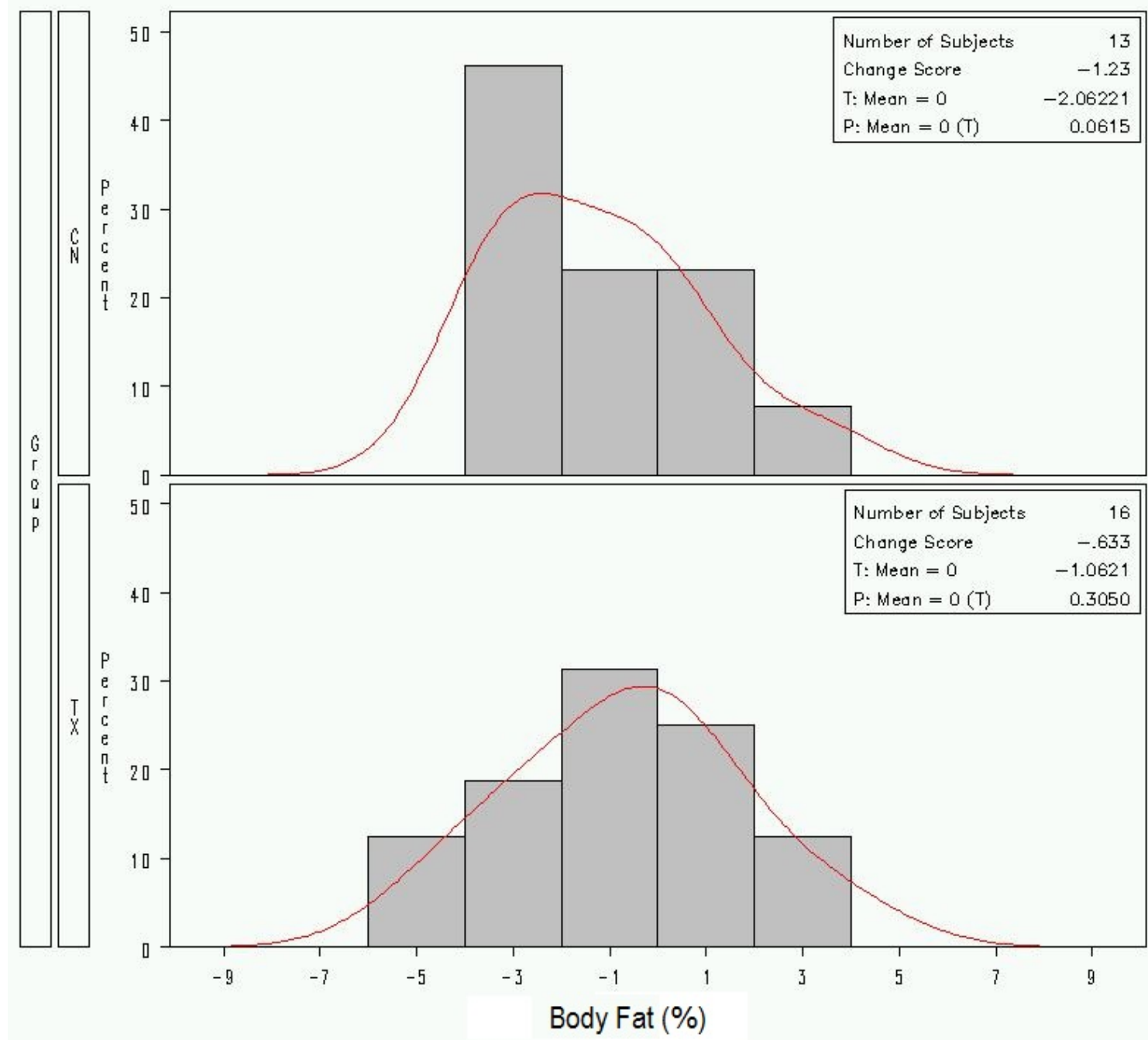

Figure 8. Comparison of Control and Treatment Group Estimated Percent Fat Pre-Post Difference Scores 


\section{Comparison of Groups}

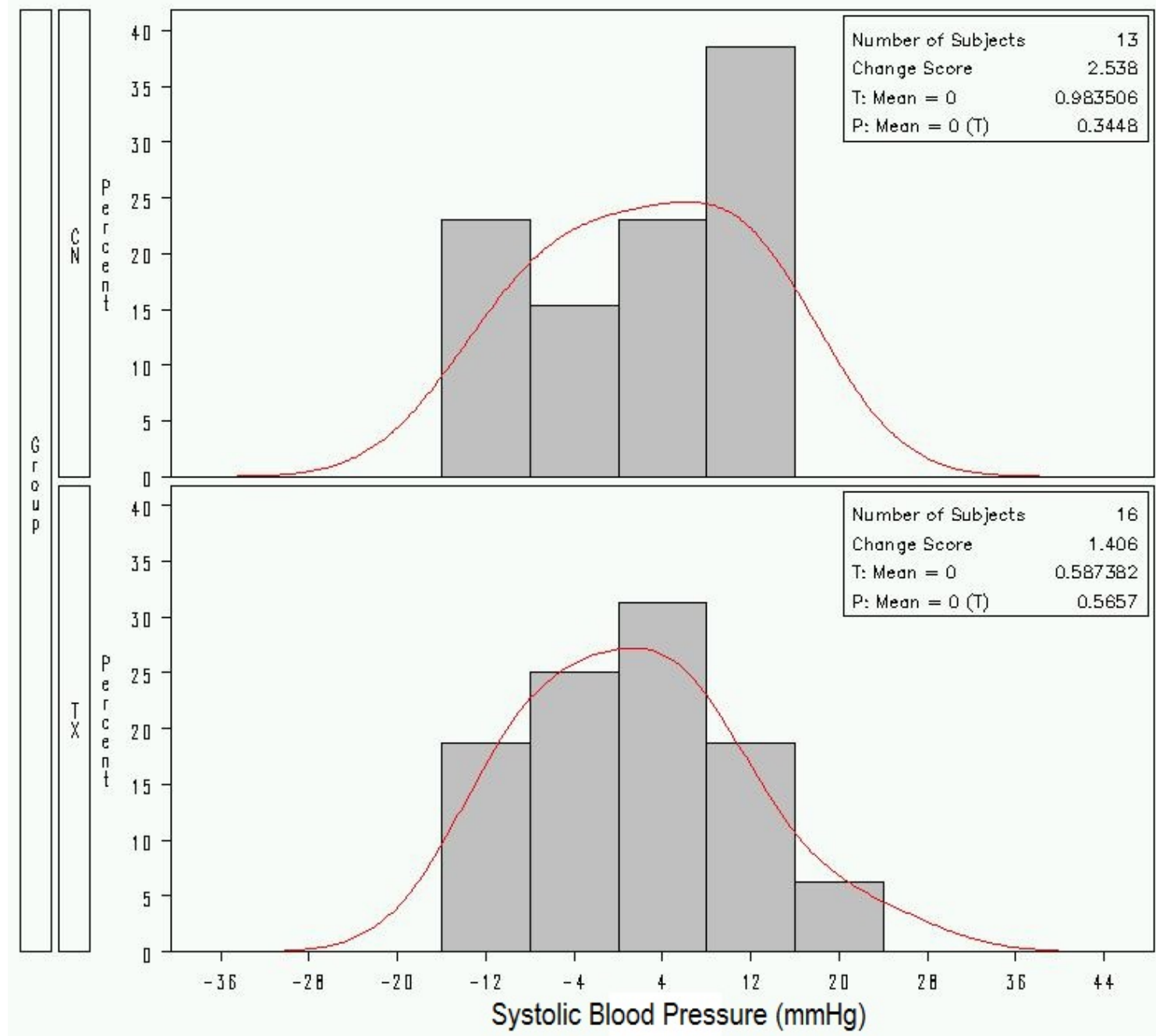

Figure 9. Comparison of Control and Treatment Group Systolic Blood Pressure PrePost Difference Scores 


\section{Comparison of Groups}

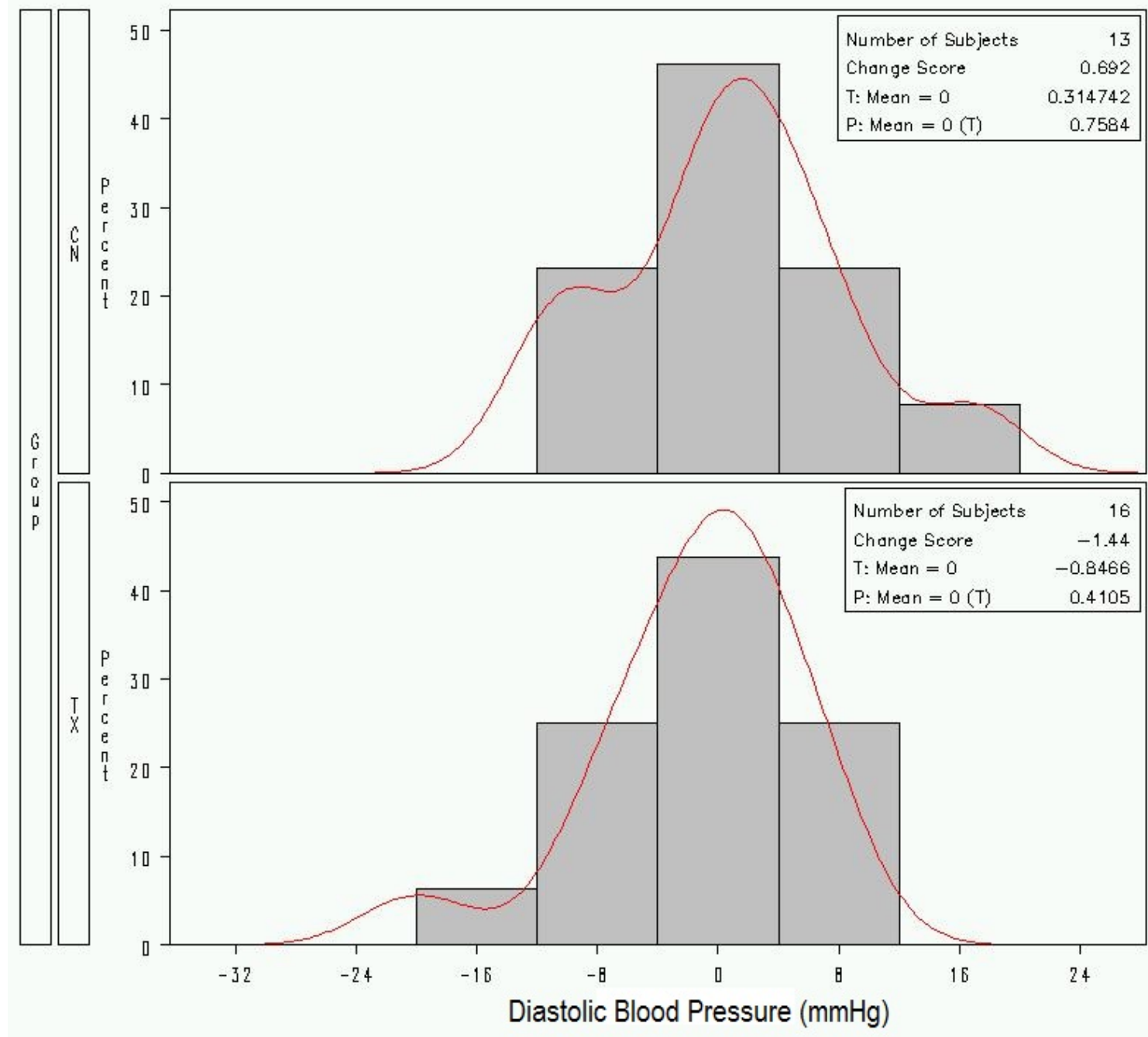

Figure 10. Comparison of Control and Treatment Group Diastolic Blood Pressure PrePost Difference Scores 


\section{Comparison of Groups}

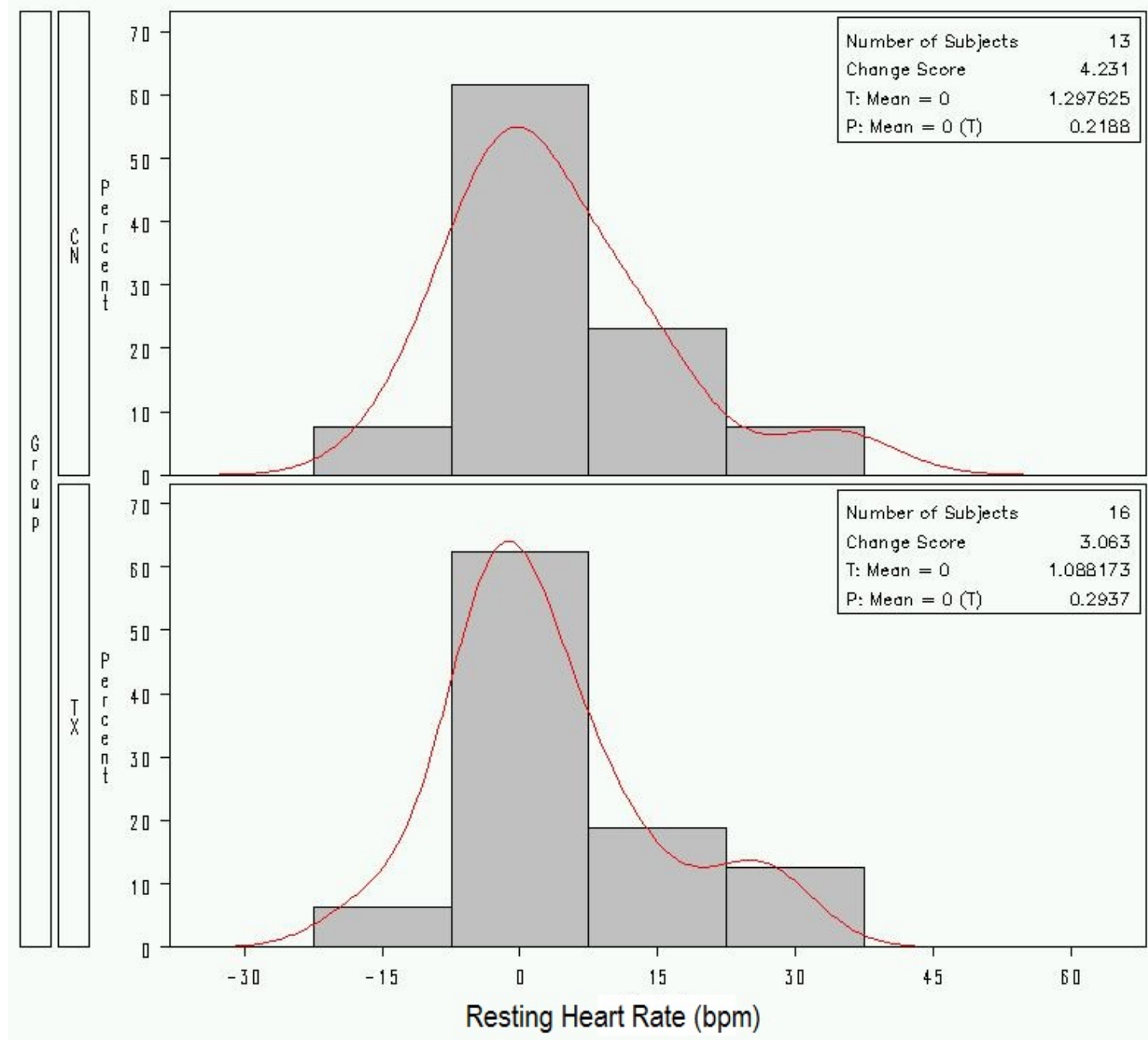

Figure 11. Comparison of Control and Treatment Group Resting Heart Rate Pre-Post Difference Scores 


\section{Comparison of Groups}

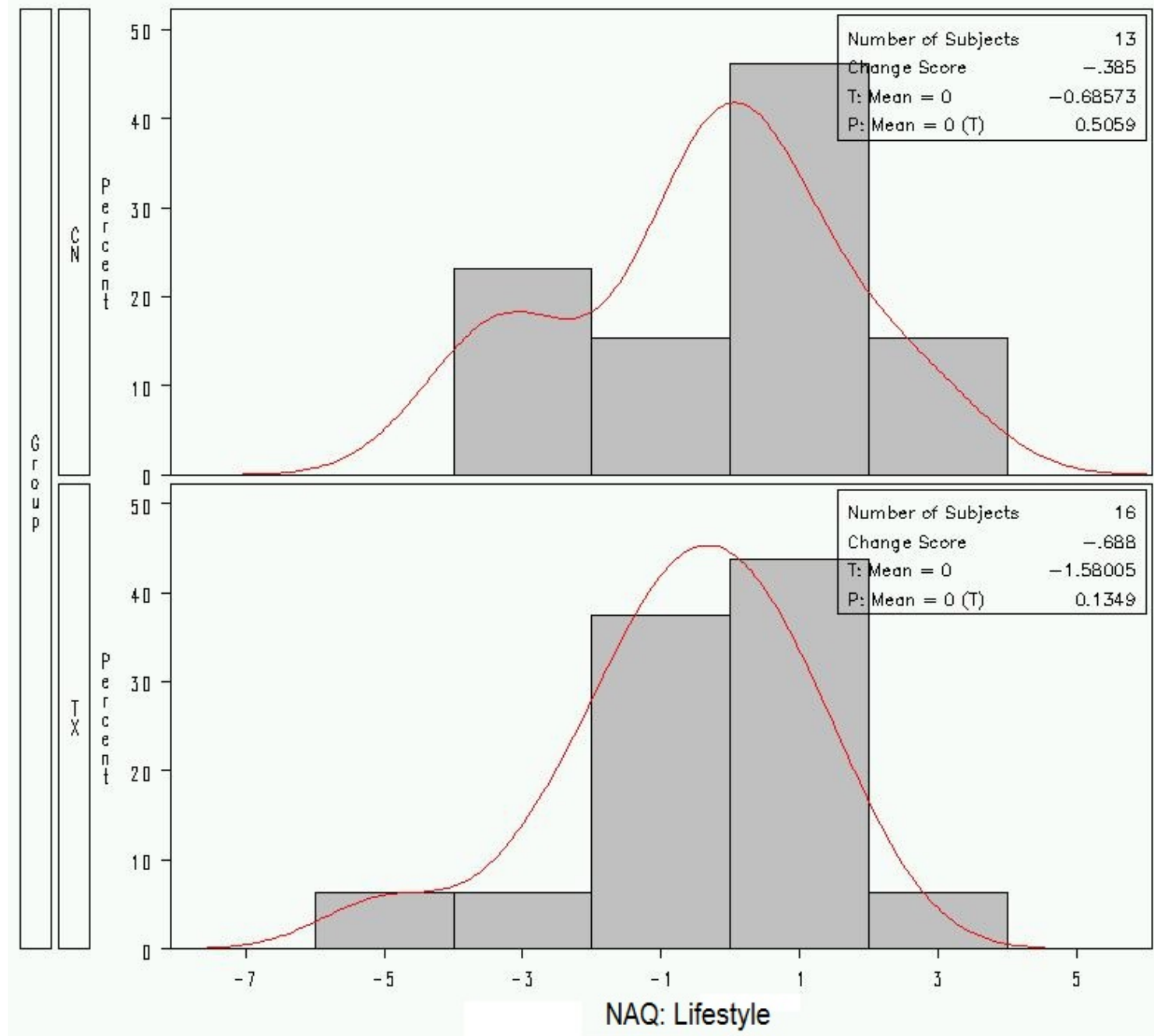

Figure 12. Comparison of Control and Treatment Group NAQ Lifestyle Subsection PrePost Difference Scores 


\section{Comparison of Groups}

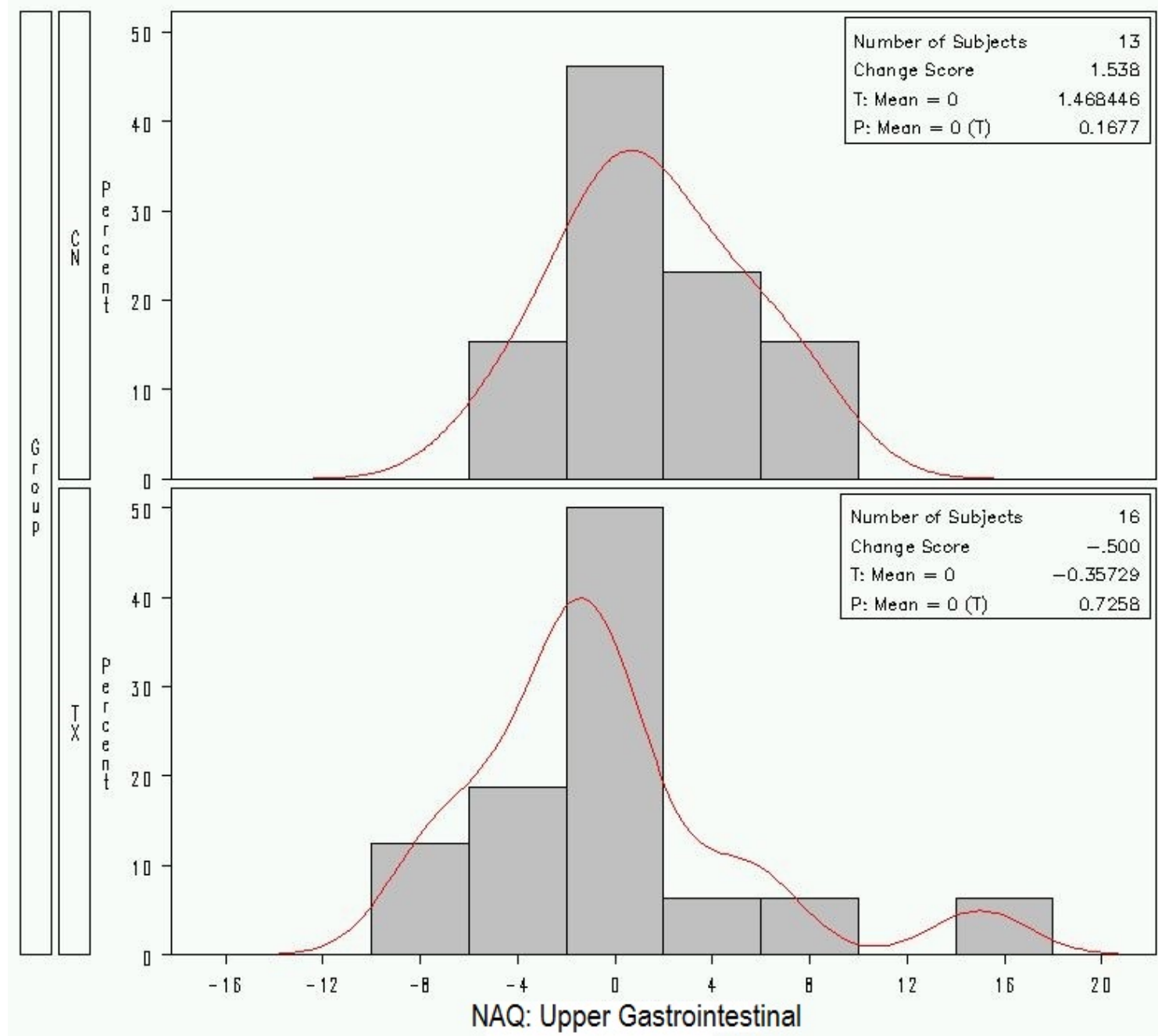

Figure 13. Comparison of Control and Treatment Group NAQ Upper GI Subsection PrePost Difference Scores 


\section{Comparison of Groups}

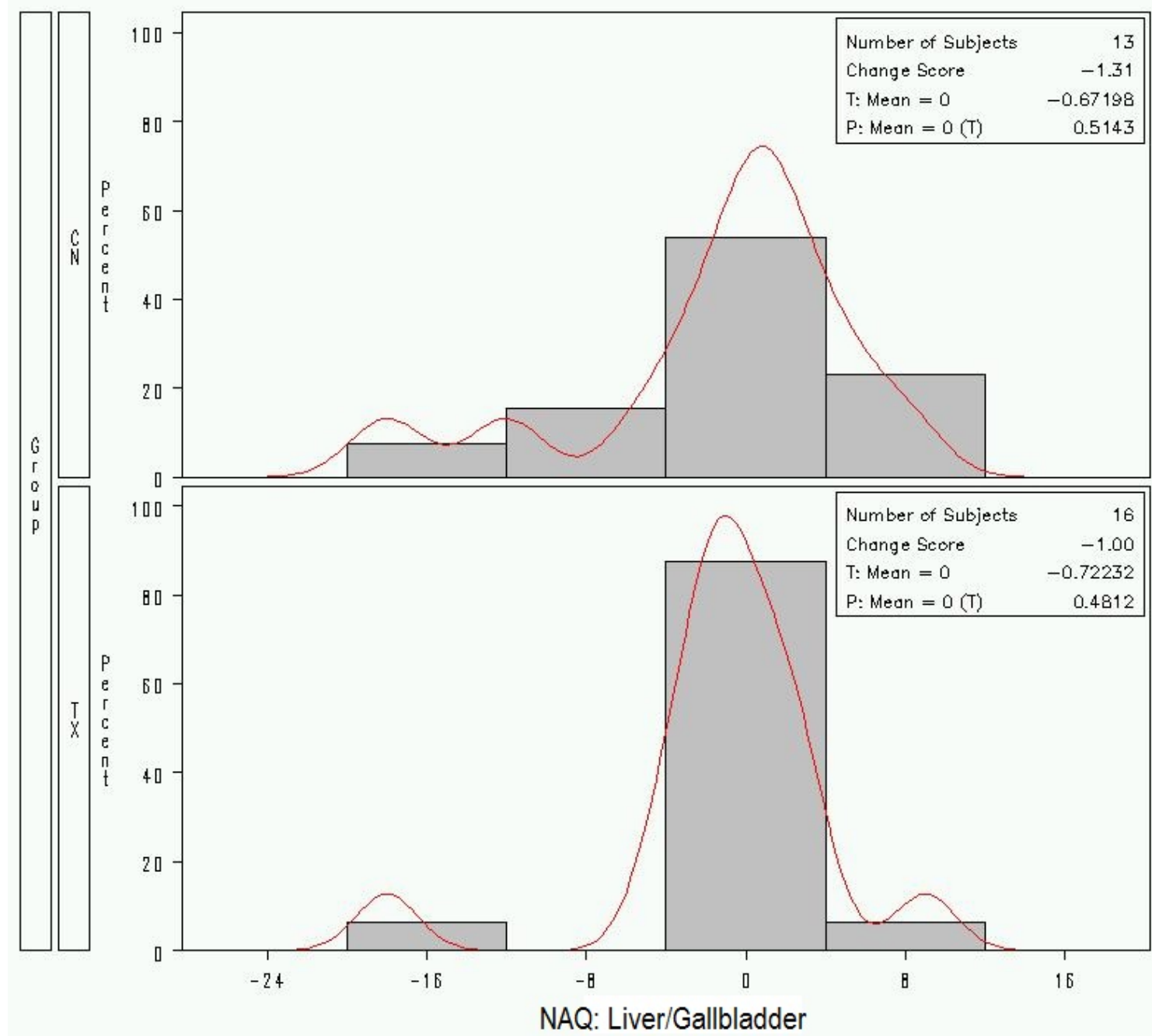

Figure 14. Comparison of Control and Treatment Group NAQ Liver/Gallbladder Subsection Pre-Post Difference Scores 


\section{Comparison of Groups}

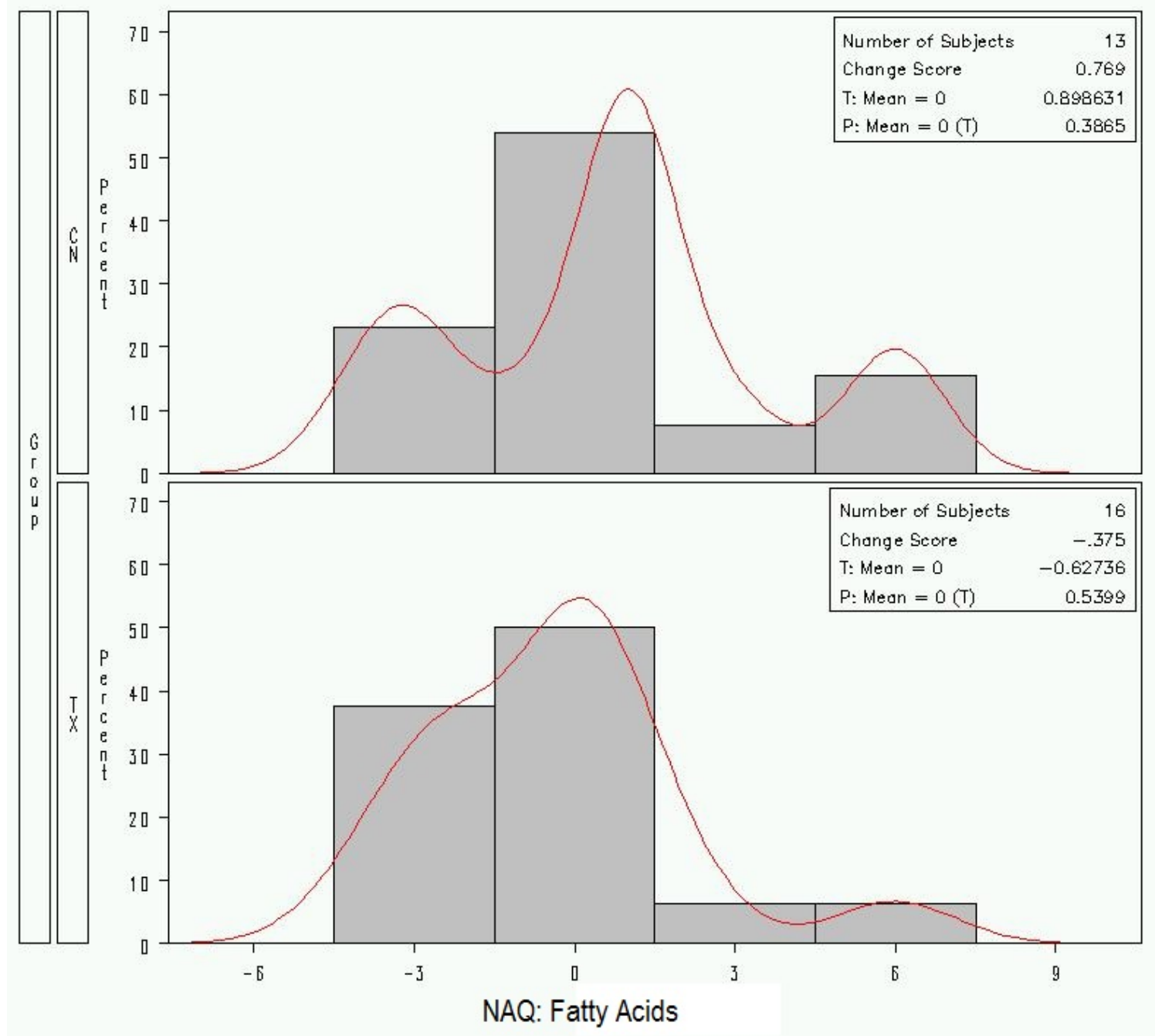

Figure 15. Comparison of Control and Treatment Group NAQ Fatty Acids Subsection Pre-Post Difference Scores 


\section{Comparison of Groups}

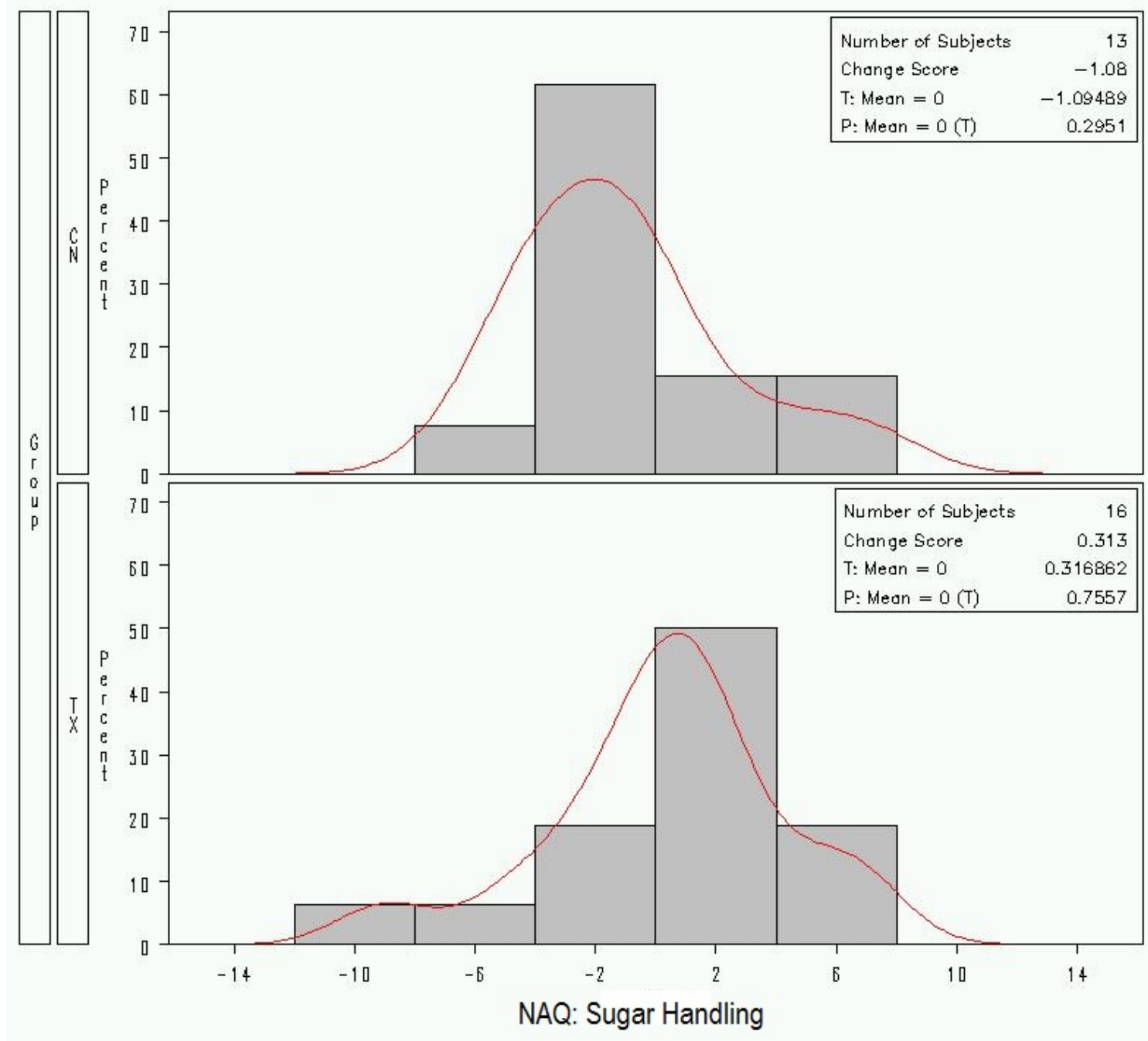

Figure 16. Comparison of Control and Treatment Group NAQ Sugar Handling Subsection Pre-Post Difference Scores 


\section{Comparison of Groups}

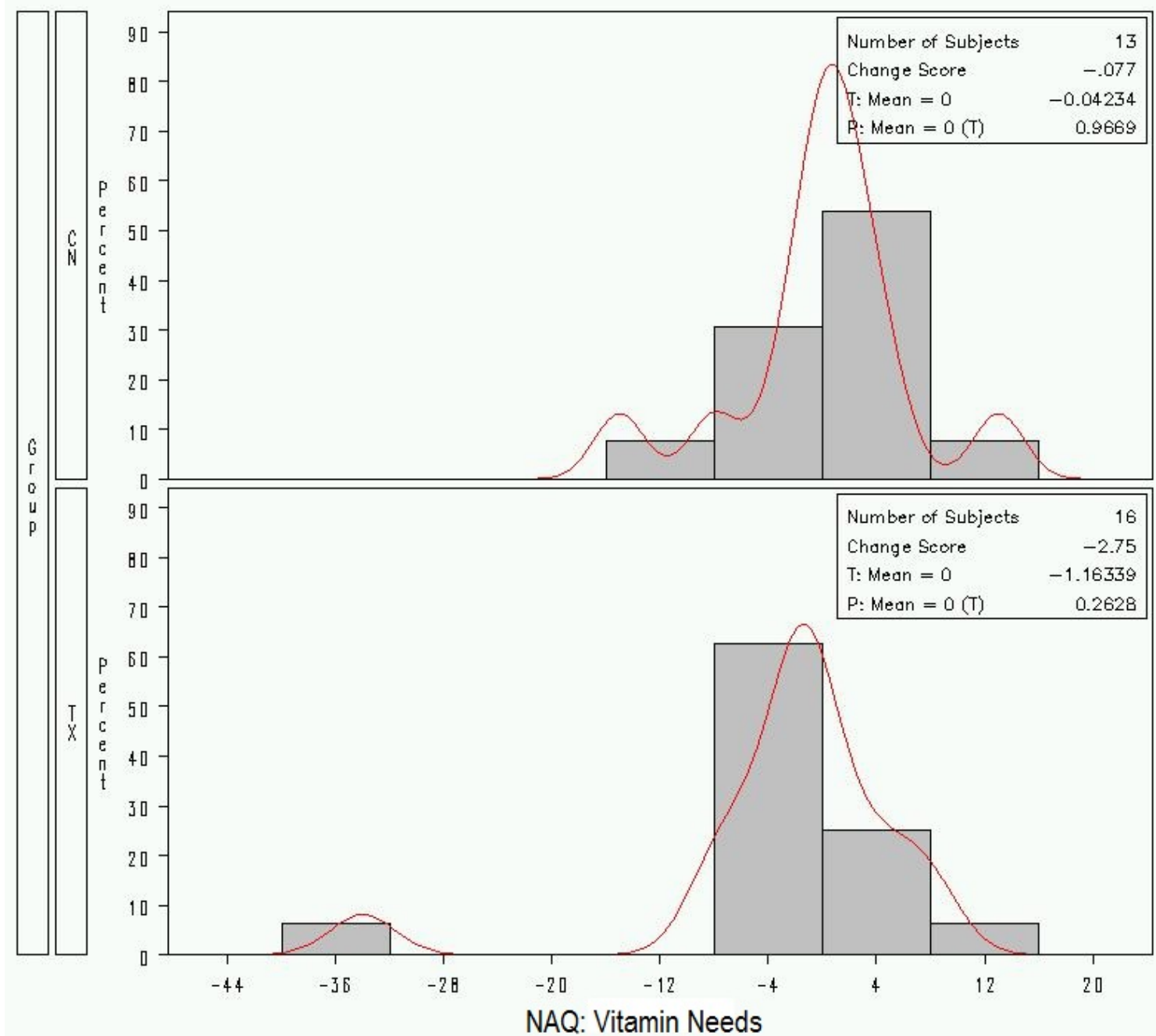

Figure 17. Comparison of Control and Treatment Group NAQ Vitamin Needs Subsection Pre-Post Difference Scores 


\section{Comparison of Groups}

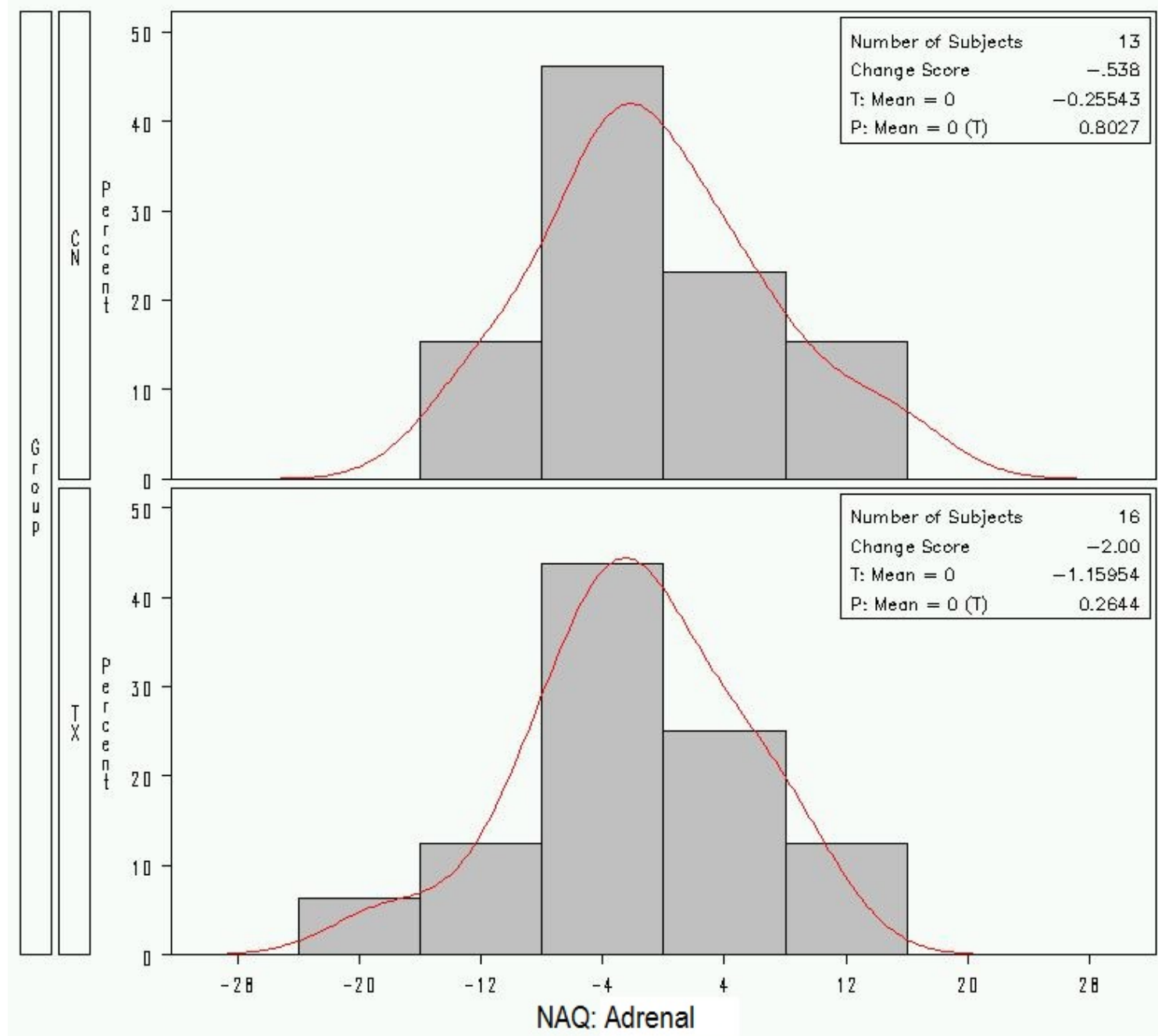

Figure 18. Comparison of Control and Treatment Group NAQ Adrenal Subsection PrePost Difference Scores 


\section{Comparison of Groups}

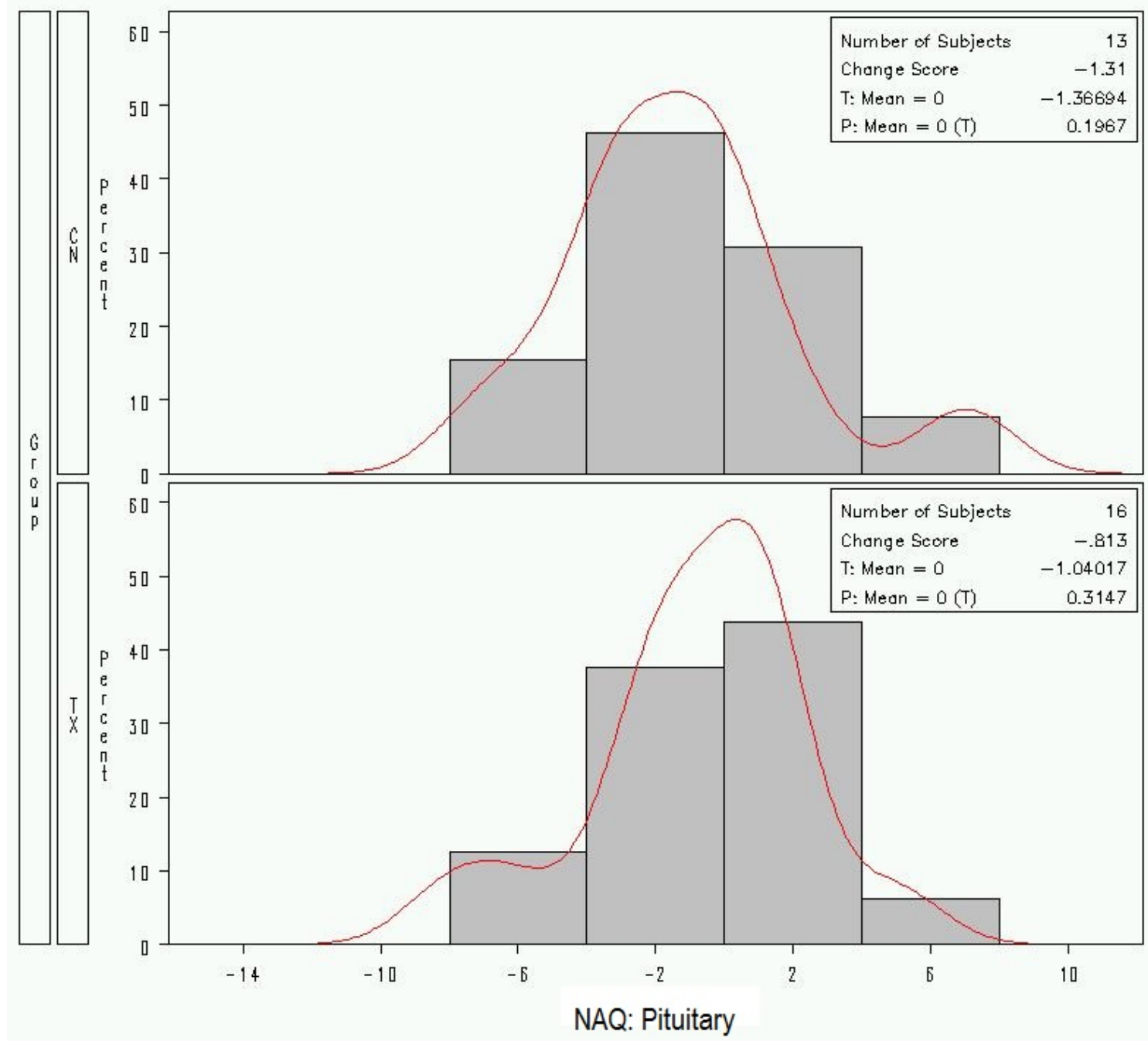

Figure 19. Comparison of Control and Treatment Group NAQ Pituitary Subsection PrePost Difference Scores 


\section{Comparison of Groups}

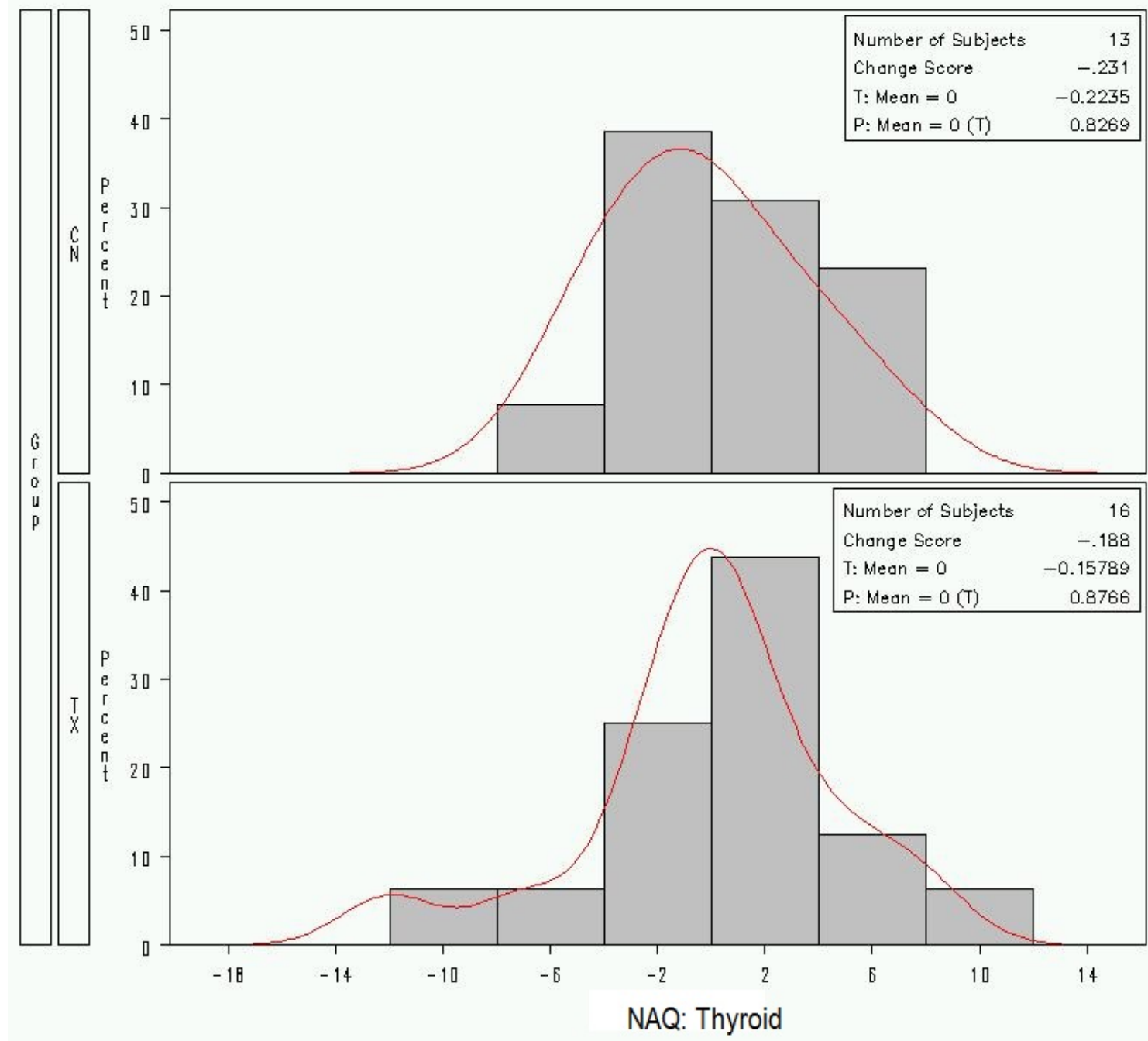

Figure 20. Comparison of Control and Treatment Group NAQ Thyroid Subsection PrePost Difference Scores 


\section{Comparison of Groups}

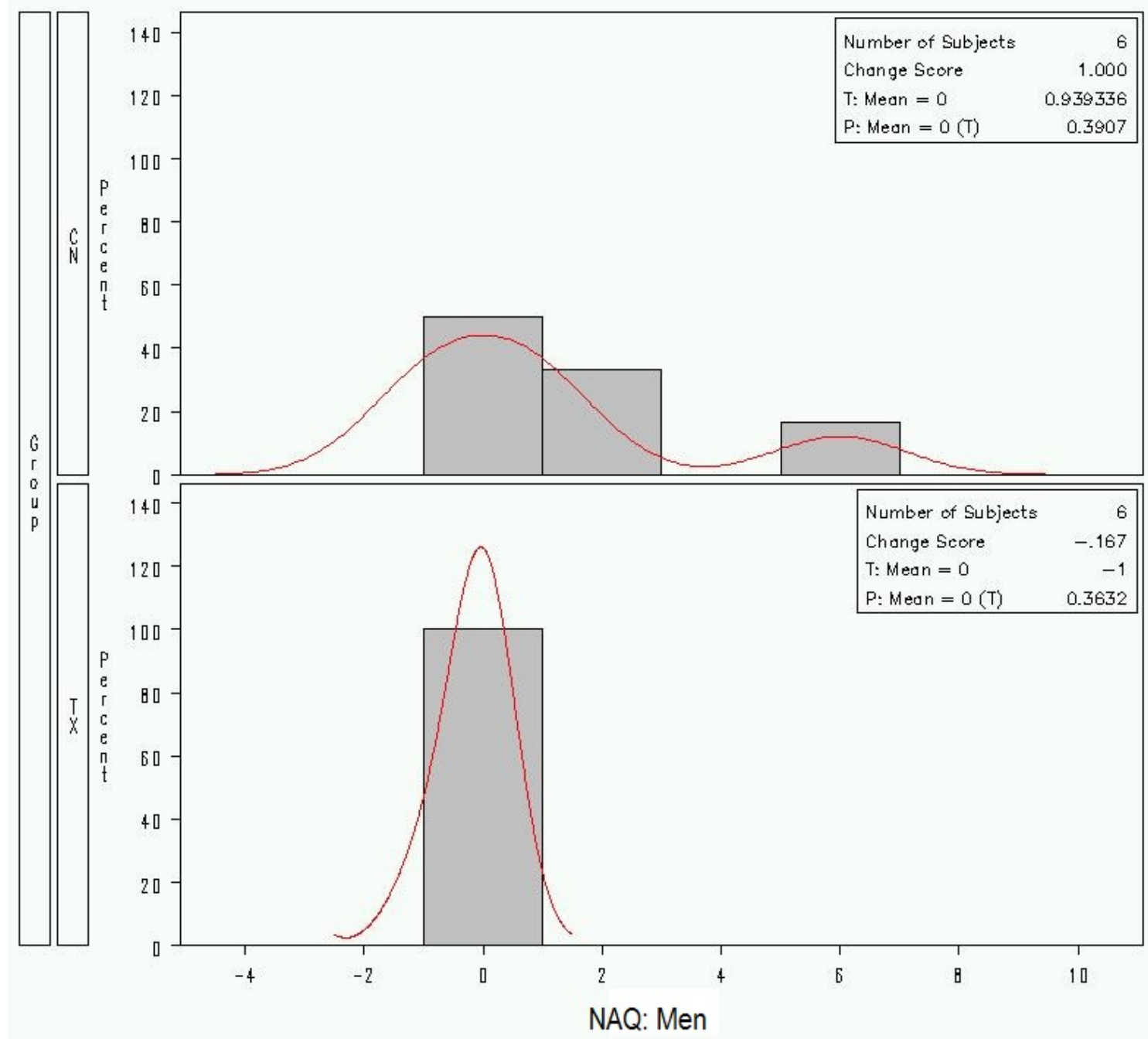

Figure 21. Comparison of Control and Treatment Group NAQ Men Subsection Pre-Post Difference Scores 


\section{Comparison of Groups}

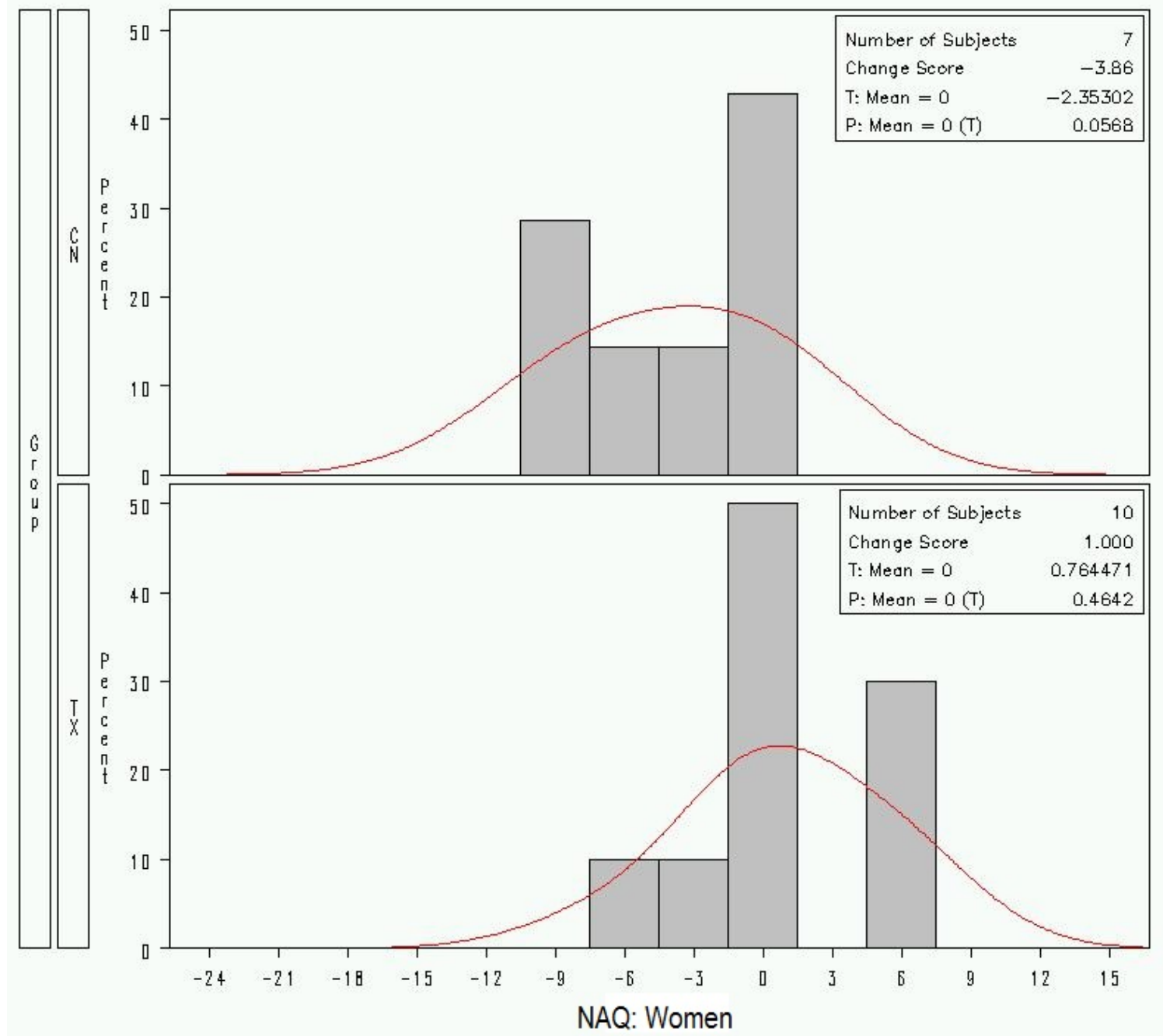

Figure 22. Comparison of Control and Treatment Group NAQ Women Subsection PrePost Difference Scores 


\section{Comparison of Groups}

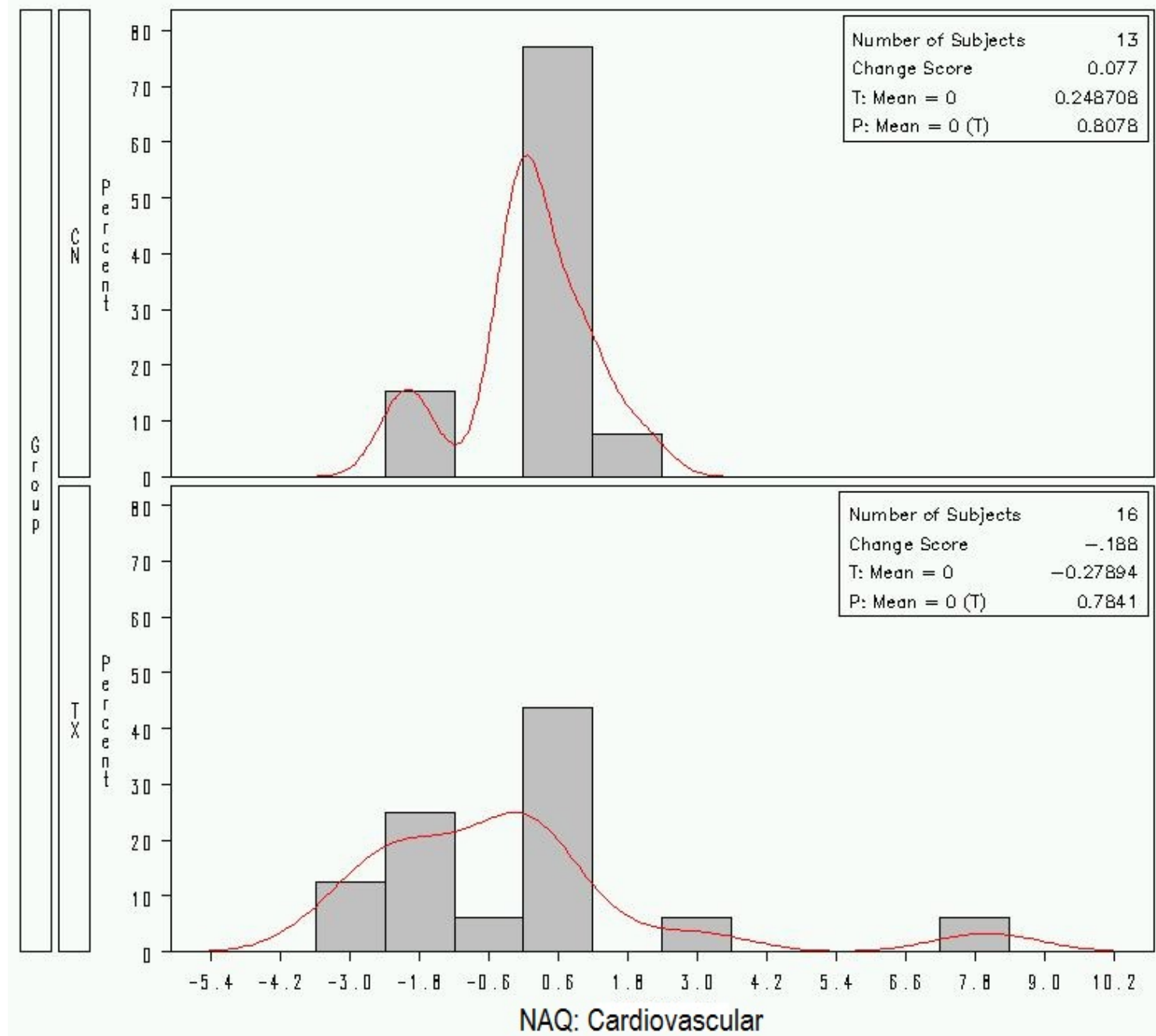

Figure 23. Comparison of Control and Treatment Group NAQ Cardio Subsection PrePost Difference Scores 


\section{Comparison of Groups}

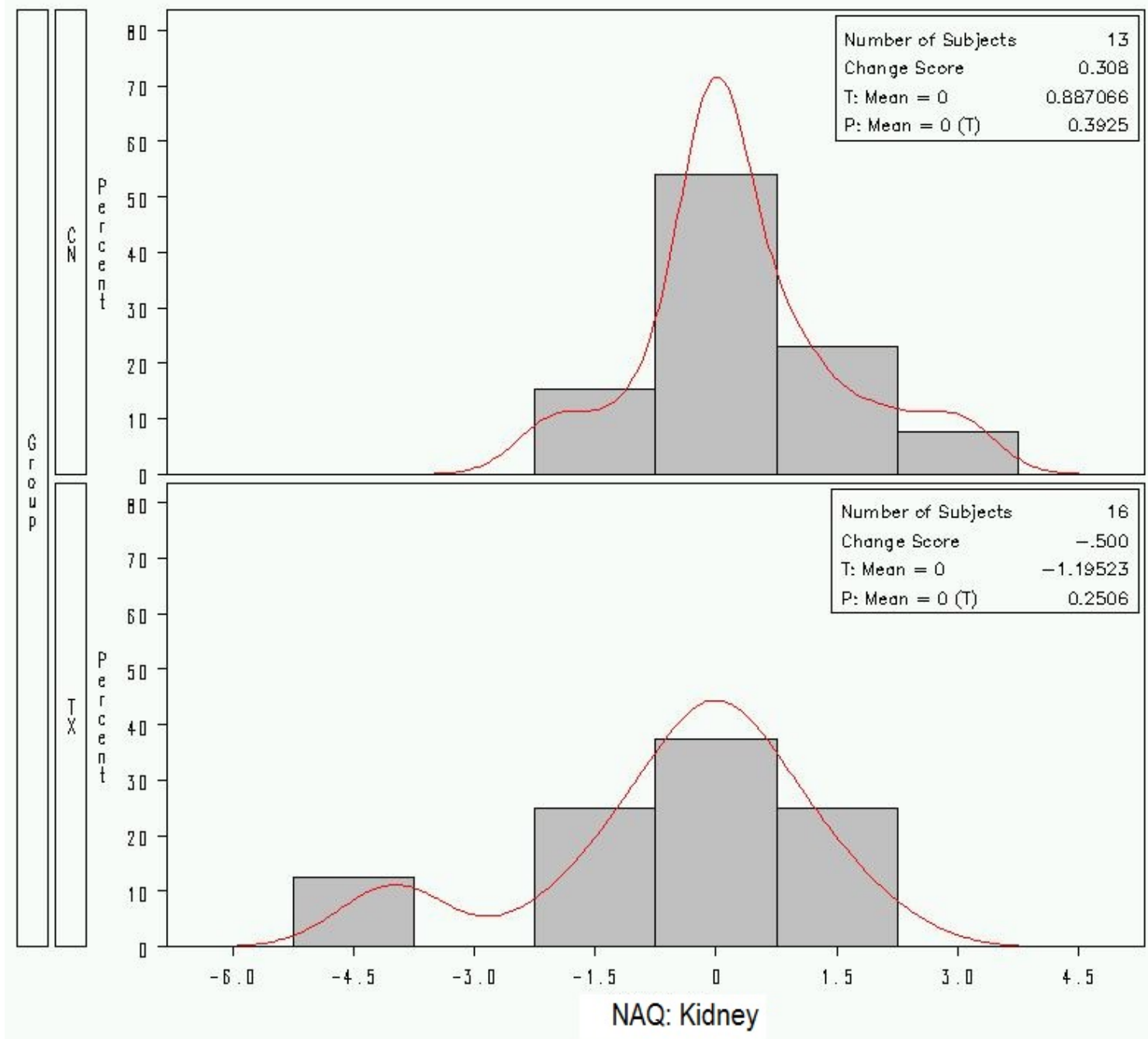

Figure 24. Comparison of Control and Treatment Group NAQ Kidney Subsection PrePost Difference Scores 


\section{Comparison of Groups}

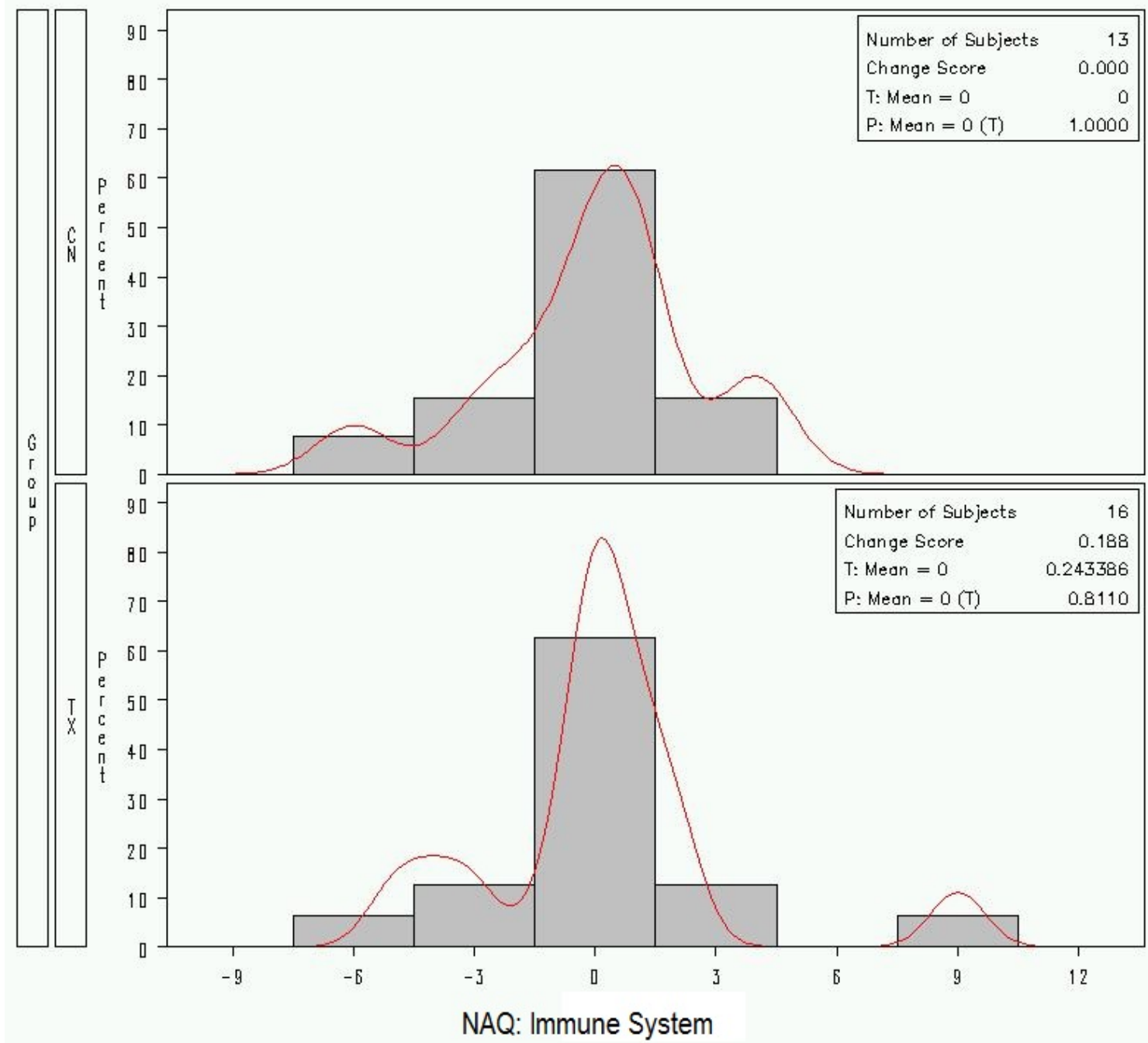

Figure 25. Comparison of Control and Treatment Group NAQ Immune System Subsection Pre-Post Difference Scores 